\title{
Three-Dimensional Vibration Analysis of Isotropic and Orthotropic Open Shells and Plates with Arbitrary Boundary Conditions
}

\author{
Guoyong Jin, Tiangui Ye, and Shuangxia Shi \\ College of Power and Energy Engineering, Harbin Engineering University, Harbin 150001, China \\ Correspondence should be addressed to Guoyong Jin; guoyongjin@hrbeu.edu.cn and Tiangui Ye; yetiangui@gmail.com
}

Received 23 December 2014; Accepted 15 April 2015

Academic Editor: Tai Thai

Copyright (C) 2015 Guoyong Jin et al. This is an open access article distributed under the Creative Commons Attribution License, which permits unrestricted use, distribution, and reproduction in any medium, provided the original work is properly cited.

\begin{abstract}
This paper presents elasticity solutions for the vibration analysis of isotropic and orthotropic open shells and plates with arbitrary boundary conditions, including spherical and cylindrical shells and rectangular plates. Vibration characteristics of the shells and plates have been obtained via a unified three-dimensional displacement-based energy formulation represented in the general shell coordinates, in which the displacement in each direction is expanded as a triplicate product of the cosine Fourier series with the addition of certain supplementary terms introduced to eliminate any possible jumps with the original displacement function and its relevant derivatives at the boundaries. All the expansion coefficients are then treated equally as independent generalized coordinates and determined by the Rayleigh-Ritz procedure. To validate the accuracy of the present method and the corresponding theoretical formulations, numerical cases have been compared against the results in the literature and those of 3D FE analysis, with excellent agreements obtained. The effects of boundary conditions, material parameters, and geometric dimensions on the frequencies are discussed as well. Finally, several 3D vibration results of isotropic and orthotropic open spherical and cylindrical shells and plates with different geometry dimensions are presented for various boundary conditions, which may be served as benchmark solutions for future researchers as well as structure designers in this field.
\end{abstract}

\section{Introduction}

In many applications and fields of modern technology, for example, civil aviation, space industry, and deep-ocean exploitation, the engineering structures often work in complex environment conditions and can be subjected to various forms of external loads, which may result in violent vibrations and lead the structures to failure. Therefore, it is of particular importance to understand the structural vibrations and reduce them through proper design to ensure a reliable, safe, and lasting structural performance. An important step in vibration design of an engineering structure is the evaluation of its vibration modal characteristics. This modal information plays a key role in the structure design and vibration suppression when subjected to dynamics excitations.

Plate and shells, such as rectangular plates and cylindrical, conical, and spherical shells, are basic structural elements of most engineering structures. A thorough understanding of their vibration characteristics is of great significance for engineers to predict the vibrations of the whole structures. Plates can be viewed as shells with zero curvature (infinite radii of curvature); thus, for simplicity, the plates are treated as a special case of shells in subsequent introductions. Recently, the vibration analysis of shells has received much attention. The literature on this subject is vast. In the literature, many of the studies are based on the thin-shell theories (such as Donnell's, Love's, and Sanders' shell theories), which are developed on the basis of the Kirchhoff-Love's simplifying assumptions [1-10]. Much more information on thin-shell theories is available in an influential monograph published by Leissa [11] in 1973. The thin-shell theories are very efficient in calculating vibration results of thin shells; however, they are highly inadequate for the analysis of even slightly thick shells due to the fact that the transverse stress and strain components are omitted. Therefore, the vibration of thick shells has conventionally been solved using the first-order 
shear deformation theories (FSDTs) and the higher-order shear deformation theories (HSDTs) [12-19]. However, shear correction factors have to be incorporated in the FSDTs to adjust the transverse shear stiffness due to the fact that the shear strains along the shell thickness are assumed to be constant. A remedy to this drawback is to use the HSDTs, which are based on the assumption of nonlinear strain variation through the shell thickness. The HSDTs, however, are still inadequate for the analysis of thick shells since the transverse normal stress and strain components are ignored in these theories. To analyze thick shells, the threedimensional (3D) theory of elasticity which accounts for all the transverse stress and strain components may be the best choice.

In recent years, vibration analysis of shells relying on the 3D theory of elasticity has attracted the attention of many researchers. The $3 \mathrm{D}$ analysis not only provides realistic results but also allows solving the whole spectrum of thick shells. Some research works in this research topic include those of Loy and Lam [20] using a layer-wise approach to study the vibration of thick cylindrical shells with simply supportedsimply supported and clamped-clamped boundary conditions. Liew and his coauthors [21-23] had developed a $p$ Ritz method to calculate vibration results of solid cylinders, spherical panels, open cylindrical shells, and curved panels. In this method, the admissible displacements of a structure are expressed in terms of sets of one- and two-dimensional orthogonal polynomial functions and solved by the Ritz method. Leissa and his coworkers [24-26] carried out a 3D method for determining the free vibration frequencies and mode shapes of thick shells with variable thickness. In this approach, displacement components in the meridional and normal directions are taken to be algebraic polynomials, respectively. Strain and kinetic energies of the shells are formulated, and upper bound values of the frequencies are obtained by minimizing the energy functional with respect to the expanded coefficients. Also, Zhou et al. [27, 28] investigated the vibration characteristics of cylindrical shells and doubly curved panels by the Chebyshev-Ritz method, in which the displacement in each direction is taken as a triplicate product of the Chebyshev polynomials in shell dimensions, multiplied by a boundary function along with a set of generalized coefficients determined by Ritz procedure. Other contributions can be seen in [29-34]. The development of researches on this topic can be seen in several monographs by Leissa and Qatu [35], Qatu [36], Reddy [37], and Saada [38] and reviews [39-41].

Compared with the above researches, it is observed that there exist a few investigations concerning 3D vibration analysis of closed shells and shallow shells with particular geometrical configurations, such as cylindrical, conical, and spherical shells. However, to the best of our knowledge, a unified 3D elasticity solution for isotropic and orthotropic open shells with arbitrary boundary conditions and variable circumferential dimensions does not seem to exist. In addition, most of the previous researches were limited to isotropic shells with a specified set of boundary conditions (such as free-fee, simply supported-simply supported, and clamped-clamped), and thus they typically require constant modifications of the trial functions and corresponding solution procedures to adapt to different boundary cases. Therefore, the use of the existing solution procedures can be easily inundated with various boundary conditions in practical applications due to the fact that there are hundreds of sets of boundary conditions for an open shell. Moreover, the open shells in the engineering applications may be of recognizable thickness-length/thickness-radius ratios and have large curvature (small radius of curvature). Therefore, a unified, reliable, and efficient method for predicting the $3 \mathrm{D}$ vibration characteristics of thick deep and shallow open shells with arbitrary boundary conditions would be highly desirable.

In view of these voids, this paper presents an endeavor to investigate the vibrations of isotropic and orthotropic open shells with arbitrary boundary conditions and variable circumferential dimensions in the framework of 3D shell theory.

In the present paper, the 3D shell theory and the energy based Rayleigh-Ritz procedure are combined to develop the elasticity solutions. Since there are many types of open shells, and it is impossible to undertake an all-encompassing survey of every case of them, therefore, the present work is focused on the open cylindrical and spherical shells, which are most frequently used in the engineering practices. Under the current framework, the displacement in each shell direction is expanded as a triplicate product of the cosine Fourier series with the addition of certain supplementary terms introduced to eliminate any possible jumps with the original displacement function and its relevant derivatives at the boundaries. All expansion coefficients are then determined by the Rayleigh-Ritz procedure. Based on the proposed method, numerical cases are presented and compared against results in the literature to validate the accuracy of this method. Effects of boundary conditions, geometric dimensions, and material properties on the natural frequencies of open shells and plates are discussed as well. Finally, several 3D vibration results of isotropic and orthotropic open cylindrical and spherical shells and plates with different geometry dimensions are presented for various boundary conditions, which may serve as benchmark solution for future researches to evaluate the new $2 \mathrm{D}$ shell theories and to compare results obtained by approximate numerical methods.

\section{Theoretical Formulations}

2.1. The Model. As shown in Figure 1(a), an open shell of meridional length $L_{\alpha}$, circumferential width $L_{\beta}$, and uniform thickness $h$ are selected as the analysis model. An orthogonal shell coordinate system $(\alpha, \beta$, and $z$ ) located in the middle surface of the shell is considered to present the geometry dimensions and its deformations, in which the coordinates $\alpha, \beta$, and $z$ are taken in the meridional, circumferential, and normal directions, respectively. $R_{\alpha}$ and $R_{\beta}$ are the mean radii of curvature in the $\alpha$ and $\beta$ directions. The displacement variations in the meridional, circumferential, and normal directions of an arbitrary point in the shell space are represented by $u, v$, and $w$, respectively. 


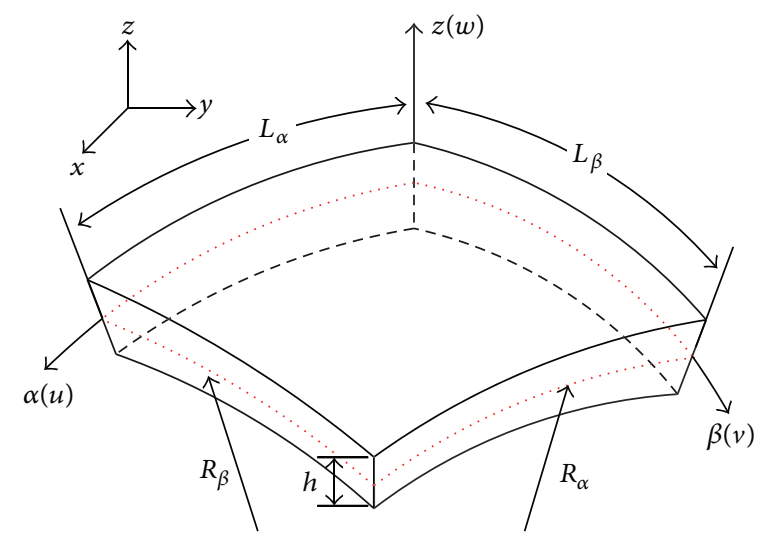

(a) Coordinate system $(\alpha, \beta$, and $z)$ of an open shell
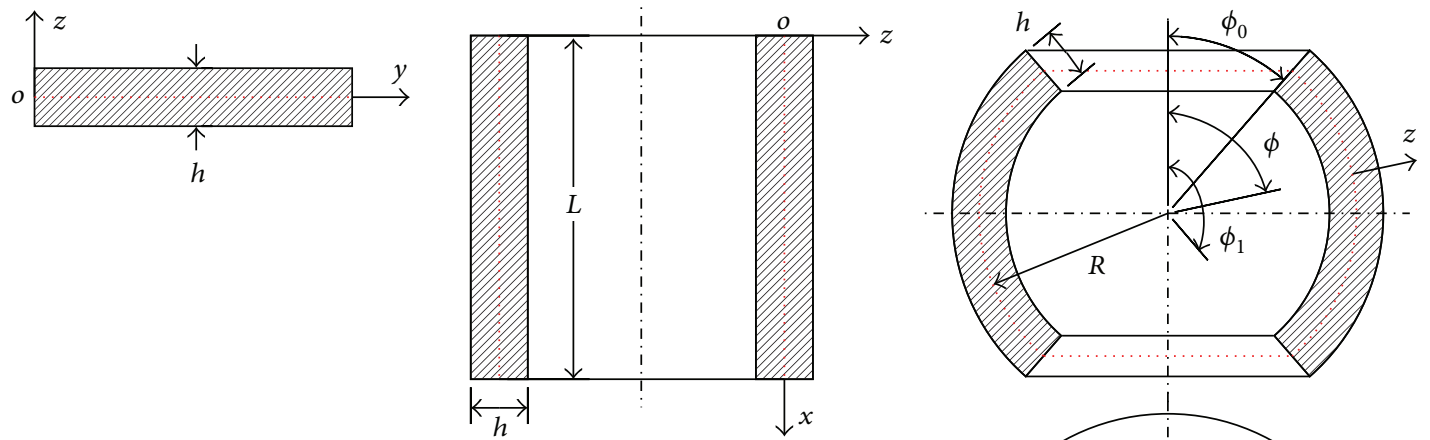

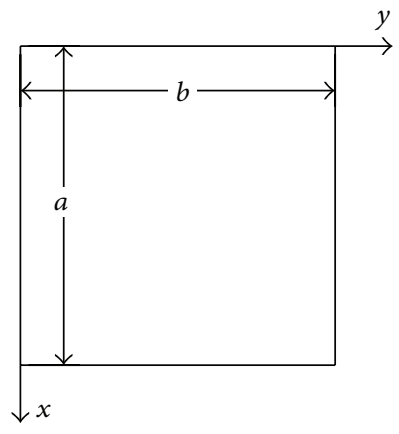

(b) Plates

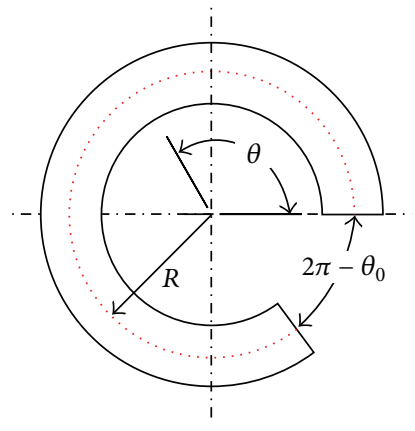

(c) Cylindrical panel

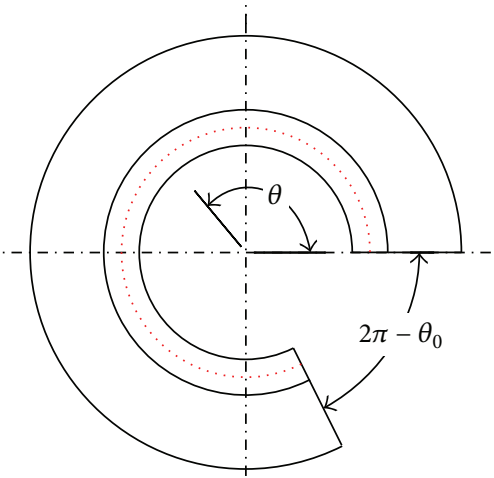

(d) Spherical panel

FIGURE 1: Geometries and coordinate systems of plates and open cylindrical and spherical shells.

2.2. Kinematic Relations and Stress Resultants. According to the three-dimensional shell theory of elasticity, the general strain-displacement relations for a shell can be defined as follows $[33,38]$ :

$$
\begin{aligned}
\varepsilon_{\alpha} & =\frac{1}{H_{\alpha}} \frac{\partial u}{\partial \alpha}+\frac{v}{H_{\alpha} H_{\beta}} \frac{\partial H_{\alpha}}{\partial \beta}+\frac{w}{H_{\alpha} H_{z}} \frac{\partial H_{\alpha}}{\partial z}, \\
\gamma_{\beta z} & =\frac{H_{z}}{H_{\beta}} \frac{\partial}{\partial \beta}\left(\frac{w}{H_{z}}\right)+\frac{H_{\beta}}{H_{z}} \frac{\partial}{\partial z}\left(\frac{v}{H_{\beta}}\right), \\
\varepsilon_{\beta} & =\frac{u}{H_{\alpha} H_{\beta}} \frac{\partial H_{\beta}}{\partial \alpha}+\frac{1}{H_{\beta}} \frac{\partial v}{\partial \beta}+\frac{w}{H_{\beta} H_{z}} \frac{\partial H_{\beta}}{\partial z}, \\
\gamma_{\alpha z} & =\frac{H_{z}}{H_{\alpha}} \frac{\partial}{\partial \alpha}\left(\frac{w}{H_{z}}\right)+\frac{H_{\alpha}}{H_{z}} \frac{\partial}{\partial z}\left(\frac{u}{H_{\alpha}}\right),
\end{aligned}
$$

$$
\begin{aligned}
\varepsilon_{z} & =\frac{u}{H_{\alpha} H_{z}} \frac{\partial H_{z}}{\partial \alpha}+\frac{v}{H_{\beta} H_{z}} \frac{\partial H_{z}}{\partial \beta}+\frac{1}{H_{z}} \frac{\partial w}{\partial z}, \\
\gamma_{\alpha \beta} & =\frac{H_{\beta}}{H_{\alpha}} \frac{\partial}{\partial \alpha}\left(\frac{v}{H_{\beta}}\right)+\frac{H_{\alpha}}{H_{\beta}} \frac{\partial}{\partial \beta}\left(\frac{u}{H_{\alpha}}\right),
\end{aligned}
$$

where $\varepsilon_{\alpha}, \varepsilon_{\beta}, \varepsilon_{z}, \gamma_{\beta z}, \gamma_{\alpha z}$, and $\gamma_{\alpha \beta}$ are the normal and shear strains. $H_{\alpha}, H_{\beta}$, and $H_{z}$ indicate the Lamé parameters; they can be derived from the rectangular coordinate system $(x, y$, and $z$ ) by the following relationships:

$$
H_{\alpha}=\sqrt{\left(\frac{\partial x}{\partial \alpha}\right)^{2}+\left(\frac{\partial y}{\partial \alpha}\right)^{2}+\left(\frac{\partial z}{\partial \alpha}\right)^{2}},
$$




$$
\begin{aligned}
& H_{\beta}=\sqrt{\left(\frac{\partial x}{\partial \beta}\right)^{2}+\left(\frac{\partial y}{\partial \beta}\right)^{2}+\left(\frac{\partial z}{\partial \beta}\right)^{2}}, \\
& H_{z}=\sqrt{\left(\frac{\partial x}{\partial z}\right)^{2}+\left(\frac{\partial y}{\partial z}\right)^{2}+\left(\frac{\partial z}{\partial z}\right)^{2}} .
\end{aligned}
$$

It should be stressed that the Lamé parameters given in (2) are determined by the adopted orthogonal curvilinear coordinate system. Consequently, the strains given in (1) depend on the adopted coordinate system as well. Since the present work is focused on the open cylindrical and spherical shells and plates, which are most frequently used in the engineering practices, therefore, under the current framework, the rectangular, cylindrical, and spherical coordinate systems are chosen to develop the theoretical formulations for the plates and open cylindrical and spherical shells, respectively. According to Figures $1(\mathrm{~b})-1(\mathrm{~d})$, the coordinates $(\alpha, \beta$, and $z$ ), geometry dimensions $\left(L_{\alpha}, L_{\beta}, R_{\alpha}\right.$, and $\left.R_{\beta}\right)$, and Lamé parameters $\left(H_{\alpha}, H_{\beta}\right.$, and $\left.H_{z}\right)$ of the plates and shells under consideration are [38]

(a) plates: $\alpha=x, \beta=y, L_{\alpha}=a, L_{\beta}=b, R_{\alpha}=R_{\beta}=\infty$, and $H_{\alpha}=H_{\beta}=H_{z}=1$,

(b) cylindrical shell: $\alpha=x, \beta=\theta, L_{\alpha}=L, L_{\beta}=R \theta_{0}$, $R_{\alpha}=\infty, R_{\beta}=R, H_{\alpha}=1, H_{\beta}=z$, and $H_{z}=1$,

(c) spherical shell: $\alpha=\phi, \beta=\theta, L_{\alpha}=R \Delta \phi, L_{\beta}=R \theta_{0}$, $R_{\alpha}=R, R_{\beta}=R, H_{\alpha}=z, H_{\beta}=z \sin \phi$, and $H_{z}=1$,

where $\theta_{0}$ is the included angle in the circumferential direction of open cylindrical and spherical shells. $\Delta \phi$ is determined by the ends of a spherical shell (i.e., $\Delta \phi=\phi_{1}-\phi_{0}$ ). The corresponding strain-displacement relations for the plates and open cylindrical and spherical shells are given in Appendix A.

For open shells and plates, the three-dimensional constitutive relations are given by Hooke's law as

$$
\begin{aligned}
& \left\{\begin{array}{c}
\sigma_{\alpha} \\
\sigma_{\beta} \\
\sigma_{z}
\end{array}\right\}=\left[\begin{array}{lll}
C_{11} & C_{12} & C_{13} \\
C_{12} & C_{22} & C_{23} \\
C_{13} & C_{23} & C_{33}
\end{array}\right]\left\{\begin{array}{l}
\varepsilon_{\alpha} \\
\left.\varepsilon_{\beta}\right\}, \\
\varepsilon_{z}
\end{array}\right\} \\
& \left\{\begin{array}{c}
\tau_{\beta z} \\
\tau_{\alpha z} \\
\tau_{\alpha \beta}
\end{array}\right\}=\left[\begin{array}{ccc}
C_{44} & 0 & 0 \\
0 & C_{55} & 0 \\
0 & 0 & C_{66}
\end{array}\right]\left\{\begin{array}{l}
\gamma_{\beta z} \\
\gamma_{\alpha z} \\
\gamma_{\alpha \beta}
\end{array}\right\},
\end{aligned}
$$

where $\sigma_{\alpha}, \sigma_{\beta}, \sigma_{z}, \tau_{\beta z}, \tau_{\alpha z}$, and $\tau_{\alpha \beta}$ are the normal and shear stresses. And the elastic stiffness coefficients $C_{i j}(i, j=1-6)$ for the orthotropic materials are given as

$$
C_{11}=\frac{E_{11}\left(1-\mu_{23} \mu_{32}\right)}{\Delta},
$$

$$
\begin{aligned}
C_{12} & =\frac{E_{11}\left(\mu_{21}+\mu_{31} \mu_{23}\right)}{\Delta}=\frac{E_{22}\left(\mu_{12}+\mu_{32} \mu_{13}\right)}{\Delta}, \\
C_{22} & =\frac{E_{22}\left(1-\mu_{31} \mu_{13}\right)}{\Delta}, \\
C_{13} & =\frac{E_{11}\left(\mu_{31}+\mu_{21} \mu_{32}\right)}{\Delta}=\frac{E_{33}\left(\mu_{13}+\mu_{12} \mu_{23}\right)}{\Delta}, \\
C_{33} & =\frac{E_{33}\left(1-\mu_{12} \mu_{21}\right)}{\Delta}, \\
C_{23} & =\frac{E_{22}\left(\mu_{32}+\mu_{12} \mu_{31}\right)}{\Delta}=\frac{E_{33}\left(\mu_{23}+\mu_{21} \mu_{32}\right)}{\Delta}, \\
C_{44} & =G_{23}, \\
C_{55} & =G_{13}, \\
C_{66} & =G_{12} \\
\Delta & =1-\mu_{12} \mu_{21}-\mu_{23} \mu_{32}-\mu_{31} \mu_{13}-2 \mu_{21} \mu_{32} \mu_{13}
\end{aligned}
$$

where $E_{11}, E_{22}$, and $E_{33}$ represent Young's moduli in the $\alpha$, $\beta$, and $z$ directions, respectively. $\mu_{i j}(i, j=1,2,3, i \neq j)$ are the corresponding Poisson's ratios. $G_{12}, G_{13}$, and $G_{23}$ indicate the shear moduli. It should be noted that there are only nine independent material parameters for orthotropic materials, that is, $E_{11}, E_{22}, E_{33}, G_{12}, G_{13}, G_{23}, \mu_{12}, \mu_{13}$, and $\mu_{23}$. The other Poisson's ratios are defined as $\mu_{i j}=E_{i i} \mu_{j i} / E_{j j}$. By letting $E_{11}=$ $E_{22}=E_{33}, \mu_{12}=\mu_{13}=\mu_{23}$ and $G_{12}=G_{13}=G_{23}=E_{11} /(2+$ $2 \mu_{12}$ ), the present formulation can be readily applied to open shells and plates made from isotropic materials.

2.3. Energy Expressions. 3D vibration analysis of structures has long been a goal of researchers in the field. Unfortunately, in most cases, it is very difficult to obtain the exact $3 \mathrm{D}$ analytical solutions of a structure. Therefore, this compels researchers to seek for approximate solutions by modern techniques such as the Rayleigh-Ritz method, DQ method, RBF method, FEM, and DSC approach. Among them, the Rayleigh-Ritz is widely adopted due to the reliability of its results and efficiency in practical calculation. Since the purpose of this work is to develop a unified, reliable, and efficient method for predicting 3D vibration characteristics of thick isotropic and orthotropic open shells and plates with arbitrary boundary conditions and variable geometry dimensions, the Rayleigh-Ritz procedure is applied in this paper. The deformation strain energy of a shell can be expressed in terms of the strains and stresses given previously as

$$
U_{V}=\frac{1}{2} \int_{0}^{L_{\alpha}} \int_{0}^{L_{\beta}} \int_{0}^{h}\left\{\varepsilon_{\alpha} \sigma_{\alpha}+\varepsilon_{\beta} \sigma_{\beta}+\varepsilon_{z} \sigma_{z}+\gamma_{\beta z} \tau_{\beta z}+\gamma_{\alpha z} \tau_{\alpha z}+\gamma_{\alpha \beta} \tau_{\alpha \beta}\right\} H_{z} H_{\beta} H_{\alpha} d z d \beta d \alpha
$$


and the kinetic energy can be written as

$$
\begin{aligned}
T & =\frac{1}{2} \\
& \cdot \int_{0}^{L_{\alpha}} \int_{0}^{L_{\beta}} \int_{0}^{h} \rho\left\{\left(\frac{\partial u}{\partial t}\right)^{2}+\left(\frac{\partial v}{\partial t}\right)^{2}+\left(\frac{\partial w}{\partial t}\right)^{2}\right\} H_{z} H_{\beta} H_{\alpha} d z d \beta d \alpha,
\end{aligned}
$$

where $\rho$ is the density of the materials. In the present work, the general restrained conditions of an open shell are realized by introducing three groups of artificial linear springs $\left(k_{u}, k_{v}\right.$, and $\left.k_{w}\right)$ at each boundary of the open shell to separately simulate the boundary forces and displacements. These boundary springs are assumed to be continuously distributed through the entire boundary domain. Therefore, arbitrary boundary conditions of the shell can be easily simulated by assigning these springs at proper stiffness. For example, the clamped boundary conditions can be realized by setting all the boundary springs to be infinitely rigid (represented by a very large number $10^{7} D$, where $D$ is the flexural stiffness $D$, defined as $\left.D=E_{11} h^{3} / 12\left(1-\mu_{12}^{2}\right)\right)$. For the purpose of convenience, symbols $k_{\psi}^{u}, k_{\psi}^{v}$, and $k_{\psi}^{w}(\psi=\alpha 0, \beta 0$, $\alpha 1$, and $\beta 1$ ) are used to indicate the stiffness (per unit area) of the boundary springs at the boundaries $\alpha=0, \beta=0, \alpha=L_{\alpha}$, and $\beta=L_{\beta}$, respectively. Therefore, the deformation strain energy about the boundary springs $\left(U_{\mathrm{sp}}\right)$ can be defined as

$$
\begin{aligned}
& U_{\text {sp }}=\frac{1}{2} \int_{0}^{L_{\beta}} \int_{0}^{h}\left\{\left.\left[k_{\alpha 0}^{u} u^{2}+k_{\alpha 0}^{v} v^{2}+k_{\alpha 0}^{w} w^{2}\right]\right|_{\alpha=0}\right. \\
& \left.+\left.\left[k_{\alpha 1}^{u} u^{2}+k_{\alpha 1}^{v} v^{2}+k_{\alpha 1}^{w} w^{2}\right]\right|_{\alpha=L_{\alpha}}\right\} H_{z} H_{\beta} d z d \beta+\frac{1}{2} \\
& \cdot \int_{0}^{L \alpha} \int_{0}^{h}\left\{\left.\left[k_{\beta 0}^{u} u^{2}+k_{\beta 0}^{v} v^{2}+k_{\beta 0}^{w} w^{2}\right]\right|_{\beta=0}\right. \\
& \left.+\left.\left[k_{\beta 1}^{u} u^{2}+k_{\beta 1}^{v} v^{2}+k_{\beta 1}^{w} w^{2}\right]\right|_{\beta=L_{\beta}}\right\} H_{z} H_{\alpha} d z d \alpha .
\end{aligned}
$$

In particular, for open cylindrical shells, the energy functions are defined as

$$
\begin{aligned}
U_{V} & =\frac{1}{2} \int_{0}^{L} \int_{0}^{\theta_{0}} \int_{R_{0}}^{R_{1}}\left\{C_{11}\left(\frac{\partial u}{\partial x}\right)^{2}+2 C_{12}\left(\frac{w}{z}+\frac{\partial v}{z \partial \theta}\right) \frac{\partial u}{\partial x}\right. \\
& +2 C_{13} \frac{\partial u}{\partial x} \frac{\partial w}{\partial z}+C_{22}\left(\frac{w}{z}+\frac{\partial v}{z \partial \theta}\right)^{2} \\
& +2 C_{23}\left(\frac{w}{z}+\frac{\partial v}{z \partial \theta}\right) \frac{\partial w}{\partial z}+C_{33}\left(\frac{\partial w}{\partial z}\right)^{2} \\
& +C_{44}\left(\frac{\partial w}{z \partial \theta}+\frac{\partial v}{\partial z}-\frac{v}{z}\right)^{2}+C_{55}\left(\frac{\partial w}{\partial x}+\frac{\partial u}{\partial z}\right)^{2} \\
& \left.+C_{66}\left(\frac{\partial u}{z \partial \theta}+\frac{\partial v}{\partial x}\right)^{2}\right\} z d z d \theta d x \\
T= & \frac{1}{2} \int_{0}^{L} \int_{0}^{\theta_{0}} \int_{R_{0}}^{R_{1}} \rho\left\{\left(\frac{\partial u}{\partial t}\right)^{2}+\left(\frac{\partial v}{\partial t}\right)^{2}+\left(\frac{\partial w}{\partial t}\right)^{2}\right\} z d z d \theta d x
\end{aligned}
$$

$$
\begin{aligned}
U_{\text {sp }} & =\frac{1}{2} \int_{0}^{\theta_{0}} \int_{R_{0}}^{R_{1}}\left\{\left.\left[k_{x 0}^{u} u^{2}+k_{x 0}^{v} v^{2}+k_{x 0}^{w} w^{2}\right]\right|_{x=0}\right. \\
& \left.+\left.\left[k_{x 1}^{u} u^{2}+k_{x 1}^{v} v^{2}+k_{x 1}^{w} w^{2}\right]\right|_{x=L}\right\} z d z d \theta \\
& +\frac{1}{2} \int_{0}^{L} \int_{R_{0}}^{R_{1}}\left\{\left.\left[k_{\theta 0}^{u} u^{2}+k_{\theta 0}^{v} v^{2}+k_{\theta 0}^{w} w^{2}\right]\right|_{\theta=0}\right. \\
& \left.+\left.\left[k_{\theta 1}^{u} u^{2}+k_{\theta 1}^{v} v^{2}+k_{\theta 1}^{w} w^{2}\right]\right|_{\theta=\theta_{0}}\right\} d z d x
\end{aligned}
$$

and, for open spherical shells, the energy functions are defined as

$$
\begin{gathered}
U_{V}=\frac{1}{2} \int_{\phi_{0}}^{\phi_{1}} \int_{0}^{\theta_{0}} \int_{R_{0}}^{R_{1}}\left\{\frac{C_{11}}{z^{2}}\left(\frac{\partial u}{\partial \phi}+w\right)^{2}+\frac{C_{22}}{z^{2}}\left(c_{\phi} u+\frac{1}{s_{\phi}} \frac{\partial v}{\partial \theta}+w\right)^{2}\right. \\
+C_{33}\left(\frac{\partial w}{\partial z}\right)^{2}+\frac{2 C_{12}}{z^{2}}\left(\frac{\partial u}{\partial \phi}+w\right)\left(c_{\phi} u+\frac{1}{s_{\phi}} \frac{\partial v}{\partial \theta}+w\right) \\
+\frac{2 C_{13}}{z}\left(\frac{\partial u}{\partial \phi}+w\right) \frac{\partial w}{\partial z}+\frac{2 C_{23}}{z}\left(c_{\phi} u+\frac{1}{s_{\phi}} \frac{\partial v}{\partial \theta}+w\right) \frac{\partial w}{\partial z} \\
+C_{44}\left[\frac{\partial v}{\partial z}+\frac{1}{z}\left(\frac{1}{s_{\phi}} \frac{\partial w}{\partial \theta}-v\right)\right]^{2}+C_{55}\left[\frac{\partial u}{\partial z}+\frac{1}{z}\left(\frac{\partial w}{\partial \phi}-u\right)\right]^{2} \\
\left.+\frac{C_{66}}{z^{2}}\left(\frac{1}{s_{\phi}} \frac{\partial u}{\partial \theta}+\frac{\partial v}{\partial \phi}-c_{\phi} v\right)^{2}\right\} z^{2} s_{\phi} d z d \theta d \phi \\
=\frac{1}{2} \int_{\phi_{0}}^{\phi_{1}} \int_{0}^{\theta_{0}} \int_{R_{0}}^{R_{1}}\left\{\rho\left\{\left(\frac{\partial u}{\partial t}\right)^{2}+\left(\frac{\partial v}{\partial t}\right)^{2}+\left(\frac{\partial w}{\partial t}\right)^{2}\right\}\right\} z^{2} s_{\phi} d z d \theta d \phi \\
U_{\mathrm{sp}}=\frac{1}{2} \int_{0}^{\theta_{0}} \int_{R_{0}}^{R_{1}}\left\{\left.\left[k_{\phi 0}^{u} u^{2}+k_{\phi 0}^{v} v^{2}+k_{\phi 0}^{w} w^{2}\right]\right|_{\phi=\phi_{0}}\right. \\
\left.+\left.\left[k_{\phi 1}^{u} u^{2}+k_{\phi 1}^{v} v^{2}+k_{\phi 1}^{w} w^{2}\right]\right|_{\phi=\phi_{1}}\right\} z s_{\phi} d z d \theta \\
+\frac{1}{2} \int_{\phi_{0}}^{\phi_{1}} \int_{R_{0}}^{R_{1}}\left\{\left.\left[k_{\theta 0}^{u} u^{2}+k_{\theta 0}^{v} v^{2}+k_{\theta 0}^{w} w^{2}\right]\right|_{\theta=0}\right. \\
\left.+\left.\left[k_{\theta 1}^{u} u^{2}+k_{\theta 1}^{v} v^{2}+k_{\theta 1}^{w} w^{2}\right]\right|_{\theta=\theta_{0}}\right\} z d z d \phi
\end{gathered}
$$

where $s_{\phi}=\sin \phi$ and $c_{\phi}=\cos \phi$.

2.4. Admissible Displacement Functions. The Raleigh-Ritz method is one of the most common approximate methods used in the vibration analysis of continuous systems. The selection of suitable admissible displacement functions is of particular importance in this method due to the fact that its convergence and accuracy highly depend on the selected admissible displacement functions. It has been always of great interest for researchers to develop a unified, accurate, and feasible admissible displacement function which can be used to determine the vibration characteristics of a structure with arbitrary boundary conditions. In the present work, an improved Fourier series proposed by Li for the vibration analysis of $1 \mathrm{D}$ [42] and 2D [43] structures with general boundary conditions is extended to investigate the 
$3 \mathrm{D}$ vibration characteristics of open shells and plates. The displacements of an open shell/plate can be written as

$$
\begin{gathered}
u(\alpha, \beta, z, t)=U(\alpha, \beta, z) e^{j \omega t}, \\
v(\alpha, \beta, z, t)=V(\alpha, \beta, z) e^{j \omega t}, \\
w(\alpha, \beta, z, t)=W(\alpha, \beta, z) e^{j \omega t},
\end{gathered}
$$

in which $\omega$ is the eigenfrequency and $j^{2}=-1 . U(\alpha, \beta, z)$, $V(\alpha, \beta, z)$, and $W(\alpha, \beta, z)$ are the $3 \mathrm{D}$ improved Fourier series expansions constructed as

$$
\begin{aligned}
U(\alpha, \beta, z) & \\
= & \sum_{m=0}^{M} \sum_{n=0}^{N} \sum_{q=0}^{Q} A_{m n q} \cos \lambda_{m} \alpha \cos \lambda_{n} \beta \cos \lambda_{q} z \\
& +\sum_{k=1}^{2} \sum_{n=0}^{N} \sum_{q=0}^{Q} A_{k n q}^{\alpha} \zeta_{k}(\alpha) \cos \lambda_{n} \beta \cos \lambda_{q} z \\
& +\sum_{k=1}^{2} \sum_{m=0}^{M} \sum_{q=0}^{Q} A_{k m q}^{\beta} \zeta_{k}(\beta) \cos \lambda_{m} \alpha \cos \lambda_{q} z \\
& +\sum_{k=1}^{2} \sum_{m=0}^{M} \sum_{n=0}^{N} A_{k m n}^{z} \zeta_{k}(z) \cos \lambda_{m} \alpha \cos \lambda_{n} \beta \\
V(\alpha, & \beta, z) \\
& \sum_{m=0}^{M} \sum_{n=0}^{N} \sum_{q=0}^{Q} B_{m n q} \cos \lambda_{m} \alpha \cos \lambda_{n} \beta \cos \lambda_{q} z \\
& +\sum_{k=1}^{2} \sum_{m=0}^{M} \sum_{n=0}^{N} B_{k m n}^{z} \zeta_{k}(z) \cos \lambda_{m} \alpha \cos \lambda_{n} \beta \\
& +\sum_{k=1}^{N} \sum_{n=0}^{Q} \sum_{q=0}^{2} B_{k n q}^{\alpha} \zeta_{k}(\alpha) \cos \lambda_{n} \beta \cos \lambda_{q} z \\
& +\sum_{k=1}^{2} \sum_{m=0}^{M} \sum_{q=0}^{Q} B_{k m q}^{\beta} \zeta_{k}(\beta) \cos \lambda_{m} \alpha \cos \lambda_{q} z
\end{aligned}
$$

$$
\begin{aligned}
& W(\alpha, \beta, z) \\
& =\sum_{m=0}^{M} \sum_{n=0}^{N} \sum_{q=0}^{Q} C_{m n q} \cos \lambda_{m} \alpha \cos \lambda_{n} \beta \cos \lambda_{q} z \\
& \quad+\sum_{k=1}^{2} \sum_{n=0}^{N} \sum_{q=0}^{Q} C_{k n q}^{\alpha} \zeta_{k}(\alpha) \cos \lambda_{n} \beta \cos \lambda_{q} z \\
& +\sum_{k=1}^{2} \sum_{m=0}^{M} \sum_{q=0}^{Q} C_{k m q}^{\beta} \zeta_{k}(\beta) \cos \lambda_{m} \alpha \cos \lambda_{q} z \\
& \quad+\sum_{k=1}^{2} \sum_{m=0}^{M} \sum_{n=0}^{N} C_{k m n}^{z} \zeta_{k}(z) \cos \lambda_{m} \alpha \cos \lambda_{n} \beta,
\end{aligned}
$$

where $\lambda_{m}=m \pi / L_{\alpha}, \lambda_{n}=n \pi / L_{\beta}$, and $\lambda_{q}=q \pi / h . M$, $N$, and $Q$ represent the truncation numbers with respect to variables $\alpha, \beta$, and $z$. The magnitude of $M, N$, and $Q$ will be determined by the convergence studies given in Section 3 . The good accuracy and fast convergence behavior of the improved Fourier series solution will be shown later. $A_{m n q}$, $B_{m n q}$, and $C_{m n q}$ are the triple cosine Fourier series expansion coefficients for the displacement components $u, v$, and $w$, respectively. $A_{k n q}^{\alpha}, A_{k m q}^{\beta}, A_{k m n}^{z}, B_{k n q}^{\alpha}, B_{k m q}^{\beta}, B_{k m n}^{z}, C_{k n q}^{\alpha}, C_{k m q}^{\beta}$, and $C_{k m n}^{z}$ are the corresponding expansion coefficients of the auxiliary functions. Each coefficient is treated equally as an independent generalized coordinate and will be determined by performing the Rayleigh-Ritz procedure in future. $\zeta_{k}(\alpha)$, $\zeta_{k}(\beta)$, and $\zeta_{k}(z)(k=1,2)$ are six auxiliary functions. The objective of introducing these auxiliary functions in each displacement is to eliminate any possible jumps with the original displacement function and its relevant derivatives at the boundaries when the displacement is expanded as a conventional triple cosine series. Thus, the function sets given in (13) are capable of representing any 3D motion of an open shell/plate with increasing accuracy as the truncated numbers are increased. It should be stressed that these jumps are not inherently related to the displacement function over the solution domain; instead, they are the artifact resulting from the Fourier series representation of the displacement solution. The detailed illustration is given in [43]. More information about the improved Fourier series can be seen in [42]. The auxiliary functions are given as

$$
\begin{aligned}
& \zeta_{1}(\alpha)=\alpha\left(\frac{\alpha}{L_{\alpha}}-1\right)^{2}, \\
& \zeta_{2}(\alpha)=\frac{\alpha^{2}}{L_{\alpha}}\left(\frac{\alpha}{L_{\alpha}}-1\right), \\
& \zeta_{1}(\beta)=\beta\left(\frac{\beta}{L_{\beta}}-1\right)^{2}, \\
& \zeta_{2}(\beta)=\frac{\beta^{2}}{L_{\beta}}\left(\frac{\beta}{L_{\beta}}-1\right), \\
& \zeta_{1}(z)=z\left(\frac{z}{h}-1\right)^{2}, \\
& \zeta_{2}(z)=\frac{z^{2}}{h}\left(\frac{z}{h}-1\right) .
\end{aligned}
$$

It can be verified that

$$
\begin{aligned}
& \zeta_{1}(0)=\zeta_{1}\left(L_{\alpha}\right)=\zeta_{2}^{\prime}\left(L_{\alpha}\right)=0, \quad \zeta_{1}^{\prime}(0)=1, \\
& \zeta_{2}(0)=\zeta_{2}\left(L_{\alpha}\right)=\zeta_{2}^{\prime}(0)=0, \quad \zeta_{2}^{\prime}\left(L_{\alpha}\right)=1 .
\end{aligned}
$$

Similar conditions can be found in the auxiliary functions related to variables $\beta$ and $z$. More detailed information about the auxiliary functions can be seen in $[43,44]$.

2.5. Solution Procedure. Since the improved Fourier series expression given in (9) is defined on the interval of 
$\left[0, L_{\alpha}\right] \otimes\left[0, L_{\beta}\right] \otimes[0, h]$, thus, linear transformations for coordinates from $R_{0}<z<R_{1}$ and $\phi_{0}<\phi<\phi_{1}$ (spherical shells) to $\bar{z} \in[0, h]$ and $\bar{\phi} \in[0, \Delta \phi]$ should be introduced for the practical programming and computing. With the energy expressions and admissible displacement functions given in the previous subsections, the remaining work is to obtain the actual displacement functions by determining the coefficients associated with the displacement functions. The Lagrangian energy functional comprising the strain and kinetic energies of the open shell/plate can be defined as

$$
L=T-U_{V}-U_{\text {sp }} .
$$

Substituting the energy expressions and the admissible displacement functions into the Lagrangian energy functional and performing the Rayleigh-Ritz operation with respect to the following undetermined coefficients:

$$
\begin{aligned}
& \frac{\partial L}{\partial \chi}=0, \\
& \chi=A_{m n q}, A_{k n q}^{\alpha}, A_{k m q}^{\beta}, A_{k m n}^{z}, B_{m n q}, B_{k n q}^{\alpha}, B_{k m q}^{\beta}, B_{k m n}^{z}, C_{m n q}, C_{k n q}^{\alpha}, C_{k m q}^{\beta}, C_{k m n}^{z},
\end{aligned}
$$

the vibration characteristic equation can be obtained and summed up as the following matrix form:

$$
\left(\mathbf{K}-\omega^{2} \mathbf{M}\right) \mathbf{G}=\mathbf{0},
$$

where $\mathbf{K}$ and $\mathbf{M}$ are, respectively, the stiffness and mass matrices. Both of them are symmetric matrices and can be written as

$$
\begin{aligned}
\mathbf{K} & =\left[\begin{array}{lll}
\mathbf{K}_{u u} & \mathbf{K}_{u v} & \mathbf{K}_{u w} \\
\mathbf{K}_{u v}^{T} & \mathbf{K}_{v v} & \mathbf{K}_{v w} \\
\mathbf{K}_{u w}^{T} & \mathbf{K}_{v w}^{T} & \mathbf{K}_{w w}
\end{array}\right], \\
\mathbf{M} & =\left[\begin{array}{ccc}
\mathbf{M}_{u u} & \mathbf{0} & \mathbf{0} \\
\mathbf{0} & \mathbf{M}_{v v} & \mathbf{0} \\
\mathbf{0} & \mathbf{0} & \mathbf{M}_{w w}
\end{array}\right] .
\end{aligned}
$$

The detailed expressions of submatrices $\mathbf{K}_{u u}, \mathbf{K}_{u v}, \mathbf{K}_{u w}, \mathbf{K}_{v v}$, $\mathbf{K}_{v w}, \mathbf{K}_{w w}, \mathbf{M}_{u u}, \mathbf{M}_{v v}$, and $\mathbf{M}_{w w}$ are given in Appendix B, respectively. $\mathbf{G}$ is the column matrix which contains, in an appropriate order, the unknown expansion coefficients that appear in the series expansions (13); namely,

$$
\mathbf{G}=\left[\mathbf{G}^{u}, \mathbf{G}^{v}, \mathbf{G}^{w}\right]^{T},
$$

where

$$
\begin{aligned}
& \mathbf{G}^{u}=\left[A_{000}, \ldots, A_{m n q}, \ldots, A_{M N Q}, A_{10}^{\alpha}, \ldots, A_{k n q}^{\alpha}, \ldots,\right. \\
& A_{2 N Q}^{\alpha}, A_{10}^{\beta}, \ldots, A_{k m q}^{\beta}, \ldots, A_{2 M Q}^{\beta}, A_{10}^{z}, \ldots, A_{k m n}^{z}, \ldots, \\
& \left.A_{2 M N}^{z}\right] \\
& \mathbf{G}^{v}=\left[B_{000}, \ldots, B_{m n q}, \ldots, B_{M N Q}, B_{10}^{\alpha}, \ldots, B_{k n q}^{\alpha}, \ldots,\right. \\
& \quad B_{2 N Q}^{\alpha}, B_{10}^{\beta}, \ldots, B_{k m q}^{\beta}, \ldots, B_{2 M Q}^{\beta}, B_{10}^{z}, \ldots, B_{k m n}^{z}, \ldots, \\
& \left.B_{2 M N}^{z}\right]
\end{aligned}
$$

$$
\begin{aligned}
& \mathbf{G}^{w}=\left[C_{000}, \ldots, C_{m n q}, \ldots, C_{M N Q}, C_{10}^{\alpha}, \ldots, C_{k n q}^{\alpha}, \ldots,\right. \\
& C_{2 N Q}^{\alpha}, C_{10}^{\beta}, \ldots, C_{k m q}^{\beta}, \ldots, C_{2 M Q}^{\beta}, C_{10}^{z}, \ldots, C_{k m n}^{z}, \ldots, \\
& \left.C_{2 M N}^{z}\right] .
\end{aligned}
$$

The natural frequencies of open shells and plates under consideration can now be solved based on a standard numerical procedure. These frequencies are upper bounds on the exact values. With the truncation numbers in (13) being increased, the current results will approach the exact solution as closely as desired. The mode shape corresponding to each frequency can be easily determined by substituting the eigenvector $\mathbf{G}$ back into the improved Fourier series expansions and solving for the ratios of coefficients. In addition, the proposed method can be readily applied to force vibration analysis of isotropic and orthotropic open shells and plates with arbitrary boundary conditions by summing the loading vector $\mathbf{F}$ on the right side of (18) and solving the standing characteristic equation.

\section{Numerical Examples and Discussions}

According to the theoretical formulations presented in Section 2, a unified computer code based on the scientific software MATLAB has been developed, which is capable of calculating vibration results of isotropic and orthotropic plates and open shells with arbitrary boundary conditions and variable circumferential dimensions. With this code, in this section, several numerical examples are presented to test the convergence, accuracy, and reliability of the proposed improved Fourier series method. In the following calculations, three types of frequently encountered boundary conditions, that is, complete free boundary $(F)$, simply supported support $(S)$, and full clamped restraint $(C)$, are considered. Taking edge $\alpha=0$, for example, these types of boundary conditions can be written in terms of boundary spring rigidities as

(1) free edge $\left(\sigma_{\alpha}=\tau_{\alpha \beta}=\tau_{\alpha z}=0\right): k_{\alpha 0}^{u}=k_{\alpha 0}^{v}=k_{\alpha 0}^{w}=0$, 
TABLE 1: Convergence of the first six nature frequencies $(\mathrm{Hz})$ of open cylindrical and spherical shells with FFFF boundary conditions.

\begin{tabular}{cccccccccccccc}
\hline \multirow{2}{*}{$h / R$} & \multirow{2}{*}{ M N } & \multicolumn{1}{c}{ Open cylindrical shells } \\
& 1 & 2 & 3 & 4 & 5 & 6 & 1 & \multicolumn{4}{c}{ Open spherical shells } \\
\hline & $13 \times 13 \times 09$ & 153.43 & 215.67 & 331.35 & 413.97 & 451.54 & 596.42 & 128.46 & 148.32 & 308.56 & 357.03 & 562.05 & 572.62 \\
& $13 \times 13 \times 10$ & 153.42 & 215.66 & 331.35 & 413.97 & 451.53 & 596.41 & 128.46 & 148.31 & 308.56 & 357.02 & 562.04 & 572.60 \\
& $13 \times 13 \times 11$ & 153.42 & 215.66 & 331.35 & 413.97 & 451.53 & 596.41 & 128.46 & 148.31 & 308.56 & 357.02 & 562.04 & 572.60 \\
0.2 & $14 \times 14 \times 09$ & 153.38 & 215.67 & 331.32 & 413.93 & 451.52 & 596.41 & 128.46 & 148.26 & 308.52 & 357.01 & 562.02 & 572.34 \\
& $14 \times 14 \times 10$ & 153.38 & 215.66 & 331.32 & 413.93 & 451.52 & 596.40 & 128.45 & 148.26 & 308.51 & 357.01 & 562.01 & 572.33 \\
& $14 \times 14 \times 11$ & 153.38 & 215.66 & 331.32 & 413.93 & 451.52 & 596.40 & 128.45 & 148.26 & 308.51 & 357.01 & 562.01 & 572.33 \\
& ANSYS & 153.38 & 215.72 & 331.35 & 413.95 & 451.55 & 596.90 & 128.48 & 148.28 & 308.47 & 357.18 & 562.08 & 572.43 \\
\hline $13 \times 13 \times 09$ & 283.52 & 398.61 & 576.28 & 594.09 & 666.63 & 982.13 & 219.22 & 260.60 & 489.01 & 571.20 & 843.44 & 844.46 \\
& $13 \times 13 \times 10$ & 283.51 & 398.60 & 576.28 & 594.08 & 666.63 & 982.13 & 219.22 & 260.60 & 489.01 & 571.20 & 843.44 & 844.45 \\
$13 \times 13 \times 11$ & 283.51 & 398.60 & 576.28 & 594.08 & 666.63 & 982.13 & 219.22 & 260.60 & 489.01 & 571.20 & 843.43 & 844.45 \\
0.4 & $14 \times 14 \times 09$ & 283.50 & 398.60 & 576.27 & 594.08 & 666.62 & 982.13 & 219.22 & 260.60 & 489.01 & 571.20 & 843.44 & 844.46 \\
& $14 \times 14 \times 10$ & 283.50 & 398.60 & 576.27 & 594.08 & 666.62 & 982.13 & 219.22 & 260.60 & 489.01 & 571.20 & 843.44 & 844.45 \\
$14 \times 14 \times 11$ & 283.50 & 398.60 & 576.27 & 594.08 & 666.61 & 982.13 & 219.22 & 260.60 & 489.01 & 571.20 & 843.43 & 844.45 \\
ANSYS & 283.78 & 399.09 & 577.12 & 594.54 & 667.51 & 982.69 & 219.54 & 261.03 & 489.88 & 572.71 & 844.97 & 846.35 \\
\hline
\end{tabular}

(2) simply supported edge $\left(\sigma_{\alpha}=0, v=w=0\right): k_{\alpha 0}^{u}=$ $0, k_{\alpha 0}^{v}=k_{\alpha 0}^{w}=10^{7} \mathrm{D}$,

(3) clamped edge $(u=v=w=0): k_{\alpha 0}^{u}=k_{\alpha 0}^{v}=k_{\alpha 0}^{w}=$ $10^{7} \mathrm{D}$

where $D$ is the bending rigidity, defined as $D=E_{11} h^{3} / 12(1-$ $\left.\mu_{12}^{2}\right)$.

The appropriateness of defining these boundary conditions in terms of boundary spring rigidities will be verified by the numerical applications given later. The present formulation can be applied to shells subjected to uniform and nonuniform elastic boundary conditions, mixed boundary conditions, and their combinations as well. For simplicity, a short letter string is adopted to describe the boundary conditions of a plate/open shell, such as FCSF which denotes that the boundaries $\alpha=0, \beta=0, \alpha=L_{\alpha}$, and $\beta=L_{\beta}$ of the structure are completely free, clamped, simply supported, and completely free, respectively (counterclockwise).

3.1. Convergence Study. Theoretically, there are infinite terms in the improved Fourier expansions given in (13); thus, the expansions should be truncated and only finite terms are considered in the actual computing. As the first example, in Table 1, convergence studies of the first six frequencies $(\mathrm{Hz})$ of completely free thick open cylindrical and spherical shells with different thickness-radius ratios are performed to check the convergence behavior of the improved Fourier solutions. Two thickness-radius ratios, that is, $h / R=0.2$ and 0.4 , are considered in the study. The two shells are assumed to be made from isotropic materials with the following material properties: $E=210 \mathrm{GPa}, \mu=0.3$, and $\rho=7800 \mathrm{~kg} / \mathrm{m}^{3}$. The geometric dimensions are $L=2 \mathrm{~m}, R=1 \mathrm{~m}$, and $\theta_{0}=2 \pi / 3$ for the cylindrical shell and $\phi_{0}=\pi / 4, \phi_{1}=3 \pi / 4, R=1 \mathrm{~m}$, and $\theta_{0}=\pi$ for the spherical one. It can be seen from Table 1 that the frequencies have converged monotonically to four significant figures. For all modes, the differences between results forms " $13 \times 13 \times 9$ " and " $14 \times 14 \times 11$ " are very small, less than $0.05 \%$. With the truncated numbers being increased, the current results will approach the exact solution as closely as desired; thus, the proposed improved Fourier method should be better understood as a method with arbitrary precision. In order to check the accuracy of the current method, results obtained by the FEM analysis (ANALYS, SOLID45, element size: $0.02 \mathrm{~m}$ ) are also listed in the table. It can be found that the two results are in good agreement. Unless otherwise stated, the improved Fourier expansions are truncated as " $14 \times 14 \times$ 11 " in all the following examples.

3.2. Plates with Arbitrary Boundary Conditions. In this subsection, we consider vibrations of isotropic and orthotropic plates. Table 2 compares the lowest seven frequency parameters $\Omega=\omega a^{2} \sqrt{\rho h / D}$ of isotropic plates with FFFF and SSSS boundary conditions. Two different thickness-length ratios, that is, $h / a=0.1$ and 0.5 , corresponding to moderately thick and thick plates are considered in the comparison. The geometry parameters and material constants used in this example are $a / b=1, E=210 \mathrm{GPa}, \mu=0.3$, and $\rho=7800 \mathrm{~kg} / \mathrm{m}^{3}$. The solutions given by Jin et al. [44] by the Ritz method with modified Fourier series and those of Liew et al. $[45,46]$ by the 3D Ritz method with general orthogonal polynomials using the Gram-Schmidt process are provided for a direct comparison. It is obvious that the present solutions agree very well with the referential data. The maximum differences between the present results and those of Jin et al. [44] and Liew et al. [45, 46] are less than $0.02 \%$ and $0.06 \%$, respectively.

We next give the results for orthotropic plates with various boundary conditions and thickness-length ratios in Table 3 and Figure 2. In Table 3, the lowest five frequency parameters $\Omega=\omega a^{2} \sqrt{\rho h / D}$ of an orthotropic rectangular plate with different combinations of boundary conditions are presented for three different thickness-length ratios; that is, $h / R=0.1,0.2$, and 0.3 . The other geometrical parameters used for the analysis are $a / b=1 / 2$. The plate is made of 
TABLE 2: Comparison of frequency parameters $\Omega=\omega a^{2} \sqrt{\rho h / D}$ of isotropic square plates with FFFF and SSSS boundary conditions $(E=$ $210 \mathrm{GPa}, \mu=0.3)$.

\begin{tabular}{|c|c|c|c|c|c|c|c|c|c|}
\hline \multirow{2}{*}{ B.C. } & \multirow{2}{*}{$h / a$} & \multirow{2}{*}{ Method } & \multicolumn{7}{|c|}{ Mode number } \\
\hline & & & 1 & 2 & 3 & 4 & 5 & 6 & 7 \\
\hline \multirow{6}{*}{ FFFF } & \multirow{3}{*}{0.1} & Reference [44] & 12.728 & 18.956 & 23.346 & 31.965 & 31.965 & 55.493 & 55.493 \\
\hline & & Reference [45] & 12.726 & 18.955 & 23.347 & 31.965 & 31.965 & 55.493 & 55.493 \\
\hline & & Present & 12.728 & 18.955 & 23.346 & 31.969 & 31.969 & 55.493 & 55.493 \\
\hline & \multirow{3}{*}{0.5} & Reference [44] & 8.7801 & 12.515 & 14.962 & 16.072 & 17.030 & 17.030 & 17.632 \\
\hline & & Reference [45] & 8.7802 & 12.515 & 14.962 & 16.073 & 17.030 & 17.030 & 17.631 \\
\hline & & Present & 8.7801 & 12.515 & 14.962 & 16.072 & 17.030 & 17.030 & 17.632 \\
\hline \multirow{6}{*}{ SSSS } & \multirow{3}{*}{0.1} & Reference [44] & 19.098 & 45.636 & 45.636 & 64.384 & 64.384 & 70.149 & 85.500 \\
\hline & & Reference [46] & 19.090 & 45.619 & 45.619 & 64.383 & 64.383 & 70.104 & 85.488 \\
\hline & & Present & 19.099 & 45.636 & 45.647 & 64.384 & 64.384 & 70.128 & 85.502 \\
\hline & \multirow{3}{*}{0.5} & Reference [44] & 12.426 & 12.877 & 12.877 & 18.210 & 23.009 & 23.009 & 25.753 \\
\hline & & Reference [46] & 12.426 & 12.877 & 12.877 & 18.210 & 23.007 & 23.007 & 25.753 \\
\hline & & Present & 12.425 & 12.877 & 12.877 & 18.210 & 23.009 & 23.009 & 25.753 \\
\hline
\end{tabular}

TABLE 3: The first five frequency parameters $\Omega=\omega a^{2} \sqrt{\rho h / D}$ of an orthotropic plate with various boundary conditions and thickness-length ratios $(a / b=1 / 2)$.

\begin{tabular}{|c|c|c|c|c|c|c|c|c|c|c|c|c|}
\hline \multirow{2}{*}{$h / a$} & \multirow{2}{*}{ Mode } & \multicolumn{11}{|c|}{ Boundary conditions } \\
\hline & & FFFF & FFFC & CFFFF & FSFS & FCFC & SFSF & SSSS & SCSC & CFCF & CSCS & CCCC \\
\hline \multirow{5}{*}{0.1} & 1 & 1.2016 & 0.1913 & 3.1286 & 0.5325 & 1.2110 & 8.0884 & 8.2286 & 8.3199 & 12.661 & 12.713 & 12.767 \\
\hline & 2 & 1.6450 & 0.9024 & 3.2604 & 1.7197 & 2.1741 & 8.1983 & 8.3304 & 8.3304 & 12.696 & 12.988 & 13.243 \\
\hline & 3 & 3.2673 & 1.1872 & 3.8097 & 2.1094 & 3.2796 & 8.3304 & 8.8058 & 9.1970 & 12.868 & 13.805 & 14.451 \\
\hline & 4 & 3.5278 & 1.6663 & 5.2189 & 3.7856 & 4.6138 & 8.6275 & 10.182 & 11.061 & 13.400 & 15.512 & 16.647 \\
\hline & 5 & 5.8822 & 2.8738 & 7.7408 & 4.1791 & 6.3016 & 9.6412 & 12.616 & 13.998 & 14.637 & 16.661 & 19.938 \\
\hline \multirow{5}{*}{0.2} & 1 & 1.1742 & 0.1900 & 2.5758 & 0.5272 & 1.1626 & 4.1652 & 4.1652 & 4.1652 & 7.4161 & 7.4588 & 7.5325 \\
\hline & 2 & 1.5490 & 0.8298 & 2.6625 & 1.5882 & 1.9269 & 5.9691 & 6.0783 & 6.1728 & 7.4365 & 7.7739 & 8.0882 \\
\hline & 3 & 3.0822 & 0.9344 & 3.1059 & 2.0326 & 3.0201 & 6.0448 & 6.5922 & 6.9602 & 7.5916 & 8.3304 & 9.3822 \\
\hline & 4 & 3.2398 & 1.1482 & 4.0386 & 2.0895 & 3.2493 & 6.3768 & 7.8472 & 8.3304 & 8.1909 & 8.7352 & 10.210 \\
\hline & 5 & 3.9204 & 2.6058 & 4.3684 & 3.4893 & 4.1349 & 7.2497 & 8.3304 & 8.5464 & 8.2659 & 10.196 & 11.435 \\
\hline \multirow{5}{*}{0.3} & 1 & 1.1337 & 0.1884 & 2.0798 & 0.5189 & 1.0997 & 2.7768 & 2.7768 & 2.7768 & 5.1645 & 5.2093 & 5.2982 \\
\hline & 2 & 1.4422 & 0.3706 & 2.1375 & 1.3930 & 1.7267 & 4.5255 & 4.6197 & 4.7182 & 5.1800 & 5.5536 & 5.8807 \\
\hline & 3 & 2.6132 & 0.7345 & 2.5347 & 1.4672 & 2.1676 & 4.5817 & 5.1096 & 5.4364 & 5.3430 & 5.5688 & 6.8086 \\
\hline & 4 & 2.8421 & 1.0972 & 2.6924 & 1.9250 & 2.7193 & 4.8655 & 5.5536 & 5.5536 & 5.5107 & 6.5703 & 7.0848 \\
\hline & 5 & 2.9387 & 1.9690 & 3.7018 & 3.1616 & 3.6066 & 5.4050 & 5.5536 & 6.7789 & 5.9915 & 6.7960 & 8.7975 \\
\hline
\end{tabular}

orthotropic material with the following material parameters: $E_{11} / E_{22}=20, E_{22}=E_{33}=10 \mathrm{GPa}, \mu_{12}=\mu_{13}=0.25$, $\mu_{23}=0.3, G_{12}=G_{13}=G_{23}=5 \mathrm{GPa}$, and $\rho=4500 \mathrm{~kg} / \mathrm{m}^{3}$. From Table 3, we can observe that the frequency parameters of the plate decrease with the thickness-radius ratio increases. Figure 2 shows the first five mode shapes of the plate with FCFC boundary conditions. The figure reveals that the mode shapes of rectangular plates varied with thickness-length ratio.

3.3. Open Cylindrical Shells with Arbitrary Boundary Conditions. In this subsection, the accuracy and reliability of the current method are validated by comparing the results with those published in the open literature and the FEM analysis firstly. Then, numerous new results of open isotropic and orthotropic cylindrical shells with different combinations of boundary conditions are presented for various thicknessradius ratios, included angles, and length-radius ratios. Also, the effects of boundary conditions, geometric dimensions, and material properties on the natural frequencies of the cylindrical shells are studied.

As the first example, comparison of the first ten frequency parameters $\Omega=\omega L \sqrt{\rho / E}$ of a thick open cylindrical shell with CFFF, SSSS, and CCCC boundary conditions is presented in Table 4 . The shell with length $L=1 \mathrm{~m}$, width $R=2 \mathrm{~m}$, thickness $h=0.5 \mathrm{~m}$, and included angle $\theta_{0}=$ $2 \arcsin (0.25)$ is assumed to be made from isotropic materials with the following material properties: $E=210 \mathrm{GPa}, \mu=0.3$, and $\rho=7800 \mathrm{~kg} / \mathrm{m}^{3}$. The comparison is performed between the current results and those provided by Liew et al. [23] by using the continuum and discrete approaches and the FE simulation (MSC/NASTRAN) based on the 3D shell theory. 

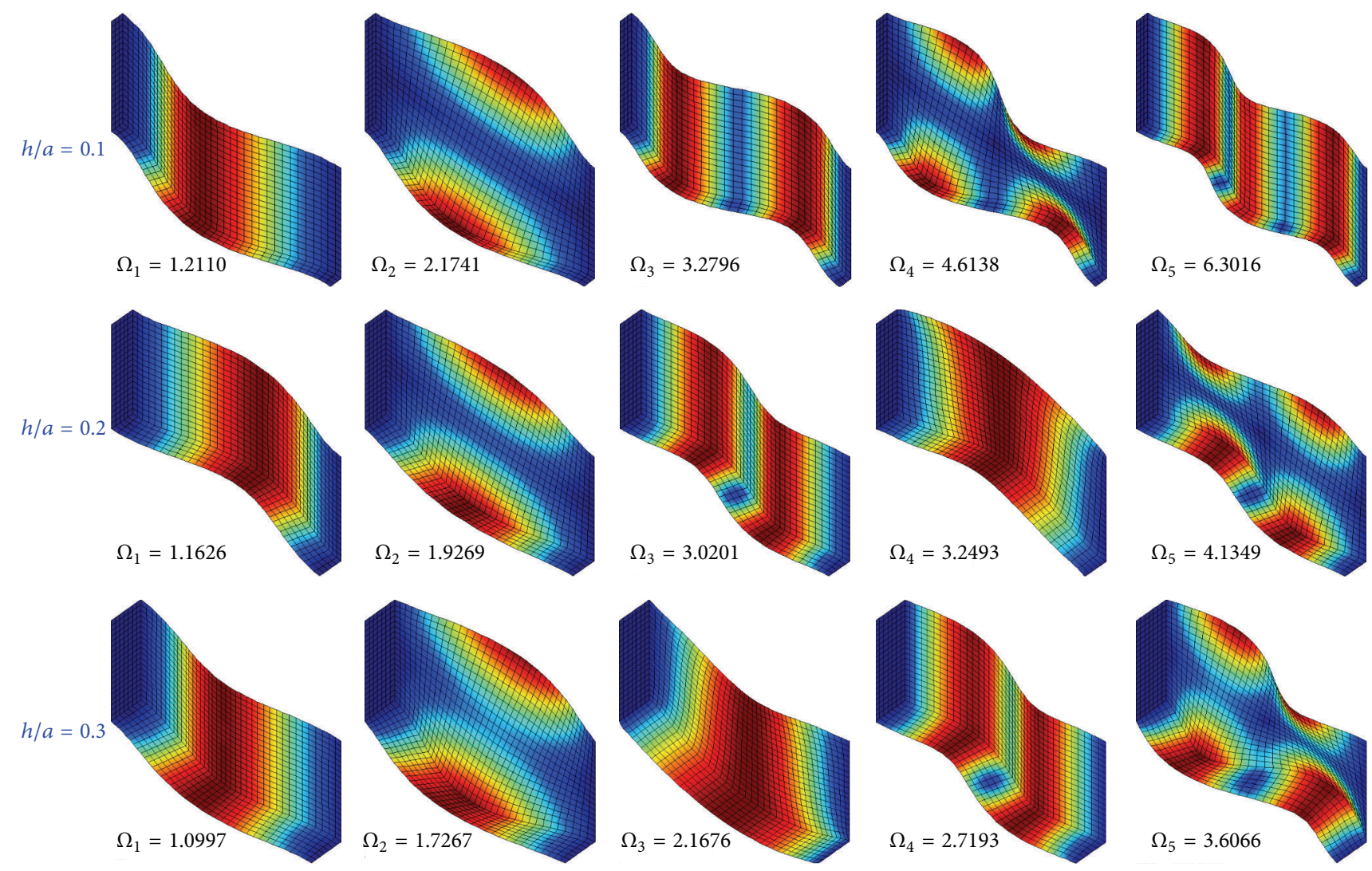

FIGURE 2: Mode shapes of an orthotropic plate with FCFC boundary conditions and various thickness-length ratios.

TABLE 4: Comparison of frequency parameters $\Omega=\omega L \sqrt{\rho / E}$ of a thick open cylindrical shell with different boundary conditions $(L=1 \mathrm{~m}$, $R / L=2, h / L=0.5, \theta_{0}=2 \sin (0.25)^{-1}, E=210 \mathrm{GPa}$, and $\left.\mu=0.3\right)$.

\begin{tabular}{|c|c|c|c|c|c|c|c|c|c|c|c|}
\hline \multirow{2}{*}{ Boundary conditions } & \multirow{2}{*}{ Theory } & \multicolumn{10}{|c|}{ Mode Number } \\
\hline & & 1 & 2 & 3 & 4 & 5 & 6 & 7 & 8 & 9 & 10 \\
\hline \multirow{3}{*}{ CFFF } & $3-\mathrm{D}[23]$ & 0.4427 & 0.6683 & 0.7822 & 1.5918 & 1.6516 & 1.7726 & 2.1988 & 2.2401 & - & - \\
\hline & FEM [23] & 0.4411 & 0.6656 & 0.7794 & 1.5858 & 1.6452 & 1.7660 & 2.1902 & 2.2317 & - & - \\
\hline & Present & 0.4431 & 0.6686 & 0.7825 & 1.5916 & 1.6531 & 1.7724 & 2.1998 & 2.2399 & 2.7542 & 3.0345 \\
\hline \multirow{3}{*}{ SSSS } & $3 \mathrm{D}[23]$ & 1.8361 & 1.9278 & 1.9483 & 2.7626 & 3.4121 & 3.4650 & 3.8276 & 3.8967 & 4.3067 & 4.3069 \\
\hline & FEM [23] & 1.8320 & - & - & 2.7565 & 3.4045 & 3.4573 & 3.8191 & 3.8880 & 4.2964 & 4.2971 \\
\hline & Present & 1.8355 & 1.9268 & 1.9473 & 2.7625 & 3.4113 & 3.4637 & 3.8257 & 3.8946 & 4.3049 & 4.3057 \\
\hline \multirow{3}{*}{ СССС } & $3 \mathrm{D}[23]$ & 2.3448 & 3.4994 & 3.6541 & 3.7476 & 3.7862 & 4.3711 & 4.7706 & 5.2640 & 5.3601 & 5.3900 \\
\hline & FEM [23] & 2.3350 & 3.4864 & 3.6405 & 3.7335 & 3.7723 & 4.3548 & 4.7528 & 5.2443 & 5.3402 & 5.3699 \\
\hline & Present & 2.3507 & 3.5063 & 3.6605 & 3.7500 & 3.7895 & 4.3705 & 4.7782 & 5.2736 & 5.3667 & 5.3992 \\
\hline
\end{tabular}

The symbol “-” represents frequency parameters that were not considered in the referential work. It is observed that the present results match well with the approximate solutions and FEM results provided by Liew et al. [23]. For all the three cases, the differences between these three results are less than $0.67 \%$. From this comparison, we can see that the presented method is accurate in predicting vibration results of open cylindrical shells with arbitrary boundary conditions. The table also reveals that it is appropriate to define the boundary conditions in terms of boundary springs.

Having validated the accuracy of the improved Fourier series solution, some further vibration results of isotropic open cylindrical shells with different combinations of boundary conditions for various geometric parameters are presented in Tables 5-7 and Figures 3-4. In Table 5, the first five frequency parameters $\Omega=\omega R \sqrt{\rho / G}$ of an isotropic deep open cylindrical shell subjected to as many as eleven combinations of boundary conditions are presented for various thickness-radius ratios. Five different thickness-radius ratios, that is, $h / R=0.1,0.2,0.3,0.4$, and 0.5 , corresponding to moderately thick to very thick open cylindrical shells, are considered in the calculation. The geometrical and material parameters used for the analysis are $R=1 \mathrm{~m}, L / R=3$, $\theta_{0}=\pi, E=210 \mathrm{GPa}, \mu=0.3$, and $\rho=7800 \mathrm{~kg} / \mathrm{m}^{3}$. From 
TABLE 5: The first five frequency parameters $\Omega=\omega R \sqrt{\rho / G}$ of an open isotropic cylindrical shell with various boundary conditions and thickness-radius ratios $\left(R=1 \mathrm{~m}, L / R=3, \theta_{0}=\pi, E=210 \mathrm{GPa}\right.$, and $\left.\mu=0.3\right)$.

\begin{tabular}{|c|c|c|c|c|c|c|c|c|c|c|c|c|}
\hline \multirow{2}{*}{$h / R$} & \multirow{2}{*}{ Mode } & \multicolumn{11}{|c|}{ Boundary conditions } \\
\hline & & FFFF & FFFC & CFFFF & FSFS & FCFC & SFSF & SSSS & SCSC & CFCF & CSCS & CCCC \\
\hline \multirow{5}{*}{0.1} & 1 & 0.0709 & 0.0211 & 0.0760 & 0.1293 & 0.2103 & 0.1669 & 0.3507 & 0.5590 & 0.2384 & 0.4713 & 0.6184 \\
\hline & 2 & 0.0888 & 0.0374 & 0.1120 & 0.1507 & 0.4467 & 0.1731 & 0.4404 & 0.5653 & 0.2597 & 0.5082 & 0.6248 \\
\hline & 3 & 0.1422 & 0.0665 & 0.1724 & 0.3626 & 0.4513 & 0.3550 & 0.6317 & 0.8884 & 0.4498 & 0.7056 & 0.9570 \\
\hline & 4 & 0.2547 & 0.0856 & 0.2685 & 0.3934 & 0.4866 & 0.4017 & 0.7440 & 0.9403 & 0.5092 & 0.7736 & 0.9661 \\
\hline & 5 & 0.3076 & 0.2253 & 0.2778 & 0.6162 & 0.7796 & 0.4178 & 0.7457 & 0.9428 & 0.5190 & 0.8340 & 1.0030 \\
\hline \multirow{5}{*}{0.2} & 1 & 0.1384 & 0.0420 & 0.1020 & 0.2542 & 0.3984 & 0.2234 & 0.4523 & 0.7116 & 0.3651 & 0.5848 & 0.7833 \\
\hline & 2 & 0.1756 & 0.0732 & 0.1667 & 0.2936 & 0.6346 & 0.2899 & 0.6368 & 0.9076 & 0.3851 & 0.7287 & 0.9652 \\
\hline & 3 & 0.2750 & 0.1307 & 0.2227 & 0.6972 & 0.7187 & 0.4402 & 0.7990 & 1.0016 & 0.6320 & 0.8631 & 1.3115 \\
\hline & 4 & 0.4669 & 0.1633 & 0.3650 & 0.7516 & 0.7958 & 0.6257 & 0.9927 & 1.2193 & 0.7024 & 1.0472 & 1.3574 \\
\hline & 5 & 0.5029 & 0.3989 & 0.4099 & 0.7858 & 1.0363 & 0.6323 & 1.0016 & 1.2566 & 0.8080 & 1.1111 & 1.5874 \\
\hline \multirow{5}{*}{0.3} & 1 & 0.2028 & 0.0625 & 0.1314 & 0.3720 & 0.5530 & 0.2753 & 0.5713 & 0.8440 & 0.4488 & 0.7125 & 0.9269 \\
\hline & 2 & 0.2595 & 0.1067 & 0.1729 & 0.4243 & 0.7671 & 0.3831 & 0.6442 & 1.0023 & 0.5181 & 0.7552 & 1.1822 \\
\hline & 3 & 0.3976 & 0.1907 & 0.3179 & 0.9237 & 0.8055 & 0.4837 & 1.0023 & 1.1203 & 0.6739 & 1.0461 & 1.5368 \\
\hline & 4 & 0.5753 & 0.2296 & 0.3694 & 0.9898 & 1.0030 & 0.7911 & 1.0461 & 1.4237 & 0.9433 & 1.1792 & 1.5915 \\
\hline & 5 & 0.6844 & 0.4928 & 0.5039 & 1.0467 & 1.1684 & 0.8068 & 1.1140 & 1.4635 & 0.9804 & 1.3171 & 1.7865 \\
\hline \multirow{5}{*}{0.4} & 1 & 0.2638 & 0.0827 & 0.1611 & 0.4812 & 0.6735 & 0.3293 & 0.6552 & 0.9578 & 0.5260 & 0.7856 & 1.0502 \\
\hline & 2 & 0.3397 & 0.1380 & 0.1782 & 0.5403 & 0.8355 & 0.4211 & 0.6884 & 1.0058 & 0.6161 & 0.8362 & 1.3129 \\
\hline & 3 & 0.5089 & 0.2469 & 0.3734 & 1.0470 & 0.8755 & 0.5745 & 1.0058 & 1.2422 & 0.7364 & 1.0468 & 1.7124 \\
\hline & 4 & 0.6770 & 0.2840 & 0.4090 & 1.0518 & 1.1187 & 0.8177 & 1.0467 & 1.5939 & 0.9687 & 1.4424 & 1.7437 \\
\hline & 5 & 0.7842 & 0.5469 & 0.5936 & 1.1198 & 1.2589 & 0.9324 & 1.3381 & 1.5954 & 1.1743 & 1.4923 & 1.9459 \\
\hline \multirow{5}{*}{0.5} & 1 & 0.3213 & 0.1024 & 0.1837 & 0.5813 & 0.7654 & 0.3826 & 0.6678 & 1.0094 & 0.5942 & 0.8148 & 1.1452 \\
\hline & 2 & 0.4155 & 0.1664 & 0.1891 & 0.6411 & 0.8500 & 0.4412 & 0.7957 & 1.0457 & 0.6758 & 0.9467 & 1.4026 \\
\hline & 3 & 0.6085 & 0.2977 & 0.3773 & 1.0471 & 0.9582 & 0.6736 & 1.0094 & 1.3213 & 0.8181 & 1.0470 & 1.8369 \\
\hline & 4 & 0.7721 & 0.3271 & 0.4932 & 1.1320 & 1.1924 & 0.8225 & 1.0470 & 1.6989 & 0.9716 & 1.6046 & 1.8548 \\
\hline & 5 & 0.8414 & 0.5867 & 0.6742 & 1.1607 & 1.3218 & 1.0096 & 1.4661 & 1.7298 & 1.3134 & 1.6287 & 1.9735 \\
\hline
\end{tabular}

the table, we can see that the frequency parameters of the shell increase with the thickness-radius ratio increases. The 3D mode shapes for the shell with FCFC boundary conditions are given in Figure 3 as well. Each of these mode shapes is determined by substituting corresponding eigenvector back into the improved Fourier series expansions and solving for the ratios of coefficients then constructed by the SURF function based on MATLAB. The shear deformations in the thickness dimensions can be seen obviously, which may not be obtained by using the $2 \mathrm{D}$ shell theories. Unlike many existing approaches limited to cylindrical shells with small curvature (shallow shells), the presented method can be used to deal with deep open shells with variable circumferential dimensions. Therefore, the first five frequency parameters $\Omega=\omega R \sqrt{\rho / G}$ of an isotropic deep open cylindrical shell with eleven combinations of boundary conditions and five different included angles are presented in Table 6. The geometrical and material properties of the shell are the same as the previous one except that the thickness-radius ratio is $h / R=$ 0.3 and the circumferential dimensions under consideration are $\theta_{0}=\pi / 3,2 \pi / 3, \pi, 4 \pi / 3$, and $5 \pi / 3$. Frequency parameters of the shell reduce with $\theta_{0}$ increases in general. In order to enhance our understanding on the vibration deformation of open cylindrical shells, some selected mode shapes of the shell with CFCF boundary conditions are given in Figure 4. It is obvious that the mode shapes of the shell varied with circumferential dimensions. For the sake of completeness, the first five frequency parameters $\Omega=\omega R \sqrt{\rho / G}$ of a deep open isotropic cylindrical shell $\left(R=1 \mathrm{~m}, h / R=0.3, \theta_{0}=\pi\right.$, $E=210 \mathrm{GPa}, \mu=0.3$, and $\rho=7800 \mathrm{~kg} / \mathrm{m}^{3}$ ) with various boundary conditions and length-radius ratios are listed in Table 7. Three different length-radius ratios performed in the calculation are $L / R=1,3$, and 5. As seen from the table, the increase of the length-radius ratio results in the decreases of the frequency parameters.

To the best of the authors' knowledge, there is no literature for 3D vibration analysis of orthotropic open cylindrical shells with arbitrary boundary conditions and variable circumferential dimensions. Therefore, some vibration results for the titled problem, which may be used as referential data for future researchers, are presented to fill this void. Unless otherwise stated, in all the following examples, open cylindrical shells under consideration are made from orthotropic materials with the following parameters: $E_{11}=200 \mathrm{GPa}$, $E_{22}=E_{33}=10 \mathrm{GPa}, \mu_{12}=\mu_{13}=0.25, \mu_{23}=0.3$, and $G_{12}=G_{13}=G_{23}=5 \mathrm{GPa}, \rho=4500 \mathrm{~kg} / \mathrm{m}^{3}$. In order to validate the accuracy of the current approach for the vibration analysis of orthotropic open cylindrical shells, 
TABLE 6: The first five frequency parameters $\Omega=\omega R \sqrt{\rho / G}$ of an open isotropic cylindrical shell with various boundary conditions and included angles $(R=1 \mathrm{~m}, L / R=3, h / R=0.3, E=210 \mathrm{GPa}$, and $\mu=0.3)$.

\begin{tabular}{|c|c|c|c|c|c|c|c|c|c|c|c|c|}
\hline \multirow{2}{*}{$\theta_{0}$} & \multirow{2}{*}{ Mode } & \multicolumn{11}{|c|}{ Boundary conditions } \\
\hline & & FFFF & FFFC & CFFFF & FSFS & FCFC & SFSF & SSSS & SCSC & CFCF & CSCS & CCCC \\
\hline \multirow{5}{*}{$\pi / 3$} & 1 & 0.3538 & 0.4457 & 0.0580 & 0.9894 & 2.4197 & 0.1595 & 1.0451 & 2.4919 & 0.3475 & 1.0451 & 2.5130 \\
\hline & 2 & 0.5316 & 0.5454 & 0.1721 & 1.0457 & 2.4308 & 0.4395 & 1.1132 & 2.6341 & 0.6014 & 1.1785 & 2.7046 \\
\hline & 3 & 0.8855 & 0.8405 & 0.2839 & 1.0563 & 2.5650 & 0.5505 & 1.5215 & 2.9448 & 0.7681 & 1.6466 & 3.0749 \\
\hline & 4 & 0.9124 & 1.2840 & 0.3436 & 1.3522 & 2.7783 & 0.6058 & 2.0902 & 2.9977 & 0.8792 & 2.0903 & 3.4482 \\
\hline & 5 & 1.1066 & 1.3494 & 0.7626 & 1.8882 & 2.9465 & 1.1108 & 2.1579 & 3.4408 & 1.2567 & 2.3133 & 3.6207 \\
\hline \multirow{5}{*}{$2 \pi / 3$} & 1 & 0.3001 & 0.1247 & 0.1016 & 0.1474 & 1.2941 & 0.2637 & 0.5085 & 1.4326 & 0.4458 & 0.6660 & 1.4879 \\
\hline & 2 & 0.5424 & 0.2076 & 0.1624 & 0.1778 & 1.3032 & 0.3108 & 1.0459 & 1.4912 & 0.4751 & 1.0459 & 1.5315 \\
\hline & 3 & 0.6280 & 0.4577 & 0.3022 & 0.9467 & 1.4013 & 0.7009 & 1.1138 & 1.5025 & 0.8703 & 1.1790 & 1.7884 \\
\hline & 4 & 0.6433 & 0.4749 & 0.4926 & 0.9897 & 1.4026 & 0.7973 & 1.1852 & 1.6759 & 0.9197 & 1.3182 & 1.9511 \\
\hline & 5 & 0.7602 & 0.6574 & 0.5502 & 1.0464 & 1.5375 & 0.7986 & 1.5025 & 1.8582 & 1.0161 & 1.6477 & 2.2660 \\
\hline \multirow{5}{*}{$\pi$} & 1 & 0.2028 & 0.0625 & 0.1314 & 0.3720 & 0.5530 & 0.2753 & 0.5713 & 0.8440 & 0.4488 & 0.7125 & 0.9269 \\
\hline & 2 & 0.2595 & 0.1067 & 0.1729 & 0.4243 & 0.7671 & 0.3831 & 0.6442 & 1.0023 & 0.5181 & 0.7552 & 1.1822 \\
\hline & 3 & 0.3976 & 0.1907 & 0.3179 & 0.9237 & 0.8055 & 0.4837 & 1.0023 & 1.1203 & 0.6739 & 1.0461 & 1.5368 \\
\hline & 4 & 0.5753 & 0.2296 & 0.3694 & 0.9898 & 1.0030 & 0.7911 & 1.0461 & 1.4237 & 0.9433 & 1.1792 & 1.5915 \\
\hline & 5 & 0.6844 & 0.4928 & 0.5039 & 1.0467 & 1.1684 & 0.8068 & 1.1140 & 1.4635 & 0.9804 & 1.3171 & 1.7865 \\
\hline \multirow{5}{*}{$4 \pi / 3$} & 1 & 0.1391 & 0.0411 & 0.1390 & 0.0377 & 0.2656 & 0.3147 & 0.5088 & 0.7306 & 0.4727 & 0.6663 & 0.8197 \\
\hline & 2 & 0.1444 & 0.0628 & 0.2031 & 0.0483 & 0.4864 & 0.3241 & 0.6708 & 0.7520 & 0.4870 & 0.7901 & 0.8867 \\
\hline & 3 & 0.2592 & 0.0996 & 0.2393 & 0.1474 & 0.5755 & 0.5652 & 0.7520 & 0.7861 & 0.7001 & 0.8348 & 1.3080 \\
\hline & 4 & 0.3729 & 0.1462 & 0.4000 & 0.1779 & 0.5980 & 0.5688 & 0.7594 & 1.2541 & 0.7540 & 1.0462 & 1.4516 \\
\hline & 5 & 0.4938 & 0.3046 & 0.4336 & 0.5078 & 0.8529 & 0.7526 & 1.0462 & 1.3212 & 0.9907 & 1.1795 & 1.4523 \\
\hline \multirow{5}{*}{$5 \pi / 3$} & 1 & 0.0878 & 0.0319 & 0.1616 & 0.0473 & 0.1398 & 0.3087 & 0.5217 & 0.6017 & 0.4753 & 0.6763 & 0.7672 \\
\hline & 2 & 0.1047 & 0.0444 & 0.1698 & 0.0484 & 0.2845 & 0.3392 & 0.5709 & 0.6474 & 0.4874 & 0.7065 & 0.7988 \\
\hline & 3 & 0.1886 & 0.0627 & 0.2808 & 0.0598 & 0.3561 & 0.4910 & 0.6017 & 0.6980 & 0.6706 & 0.8515 & 1.0052 \\
\hline & 4 & 0.2162 & 0.0984 & 0.2898 & 0.0609 & 0.4646 & 0.6021 & 0.7453 & 0.9431 & 0.7598 & 0.8886 & 1.2314 \\
\hline & 5 & 0.3302 & 0.1682 & 0.4384 & 0.2742 & 0.6681 & 0.6276 & 0.8349 & 1.1715 & 0.8233 & 1.0463 & 1.3907 \\
\hline
\end{tabular}

TABLE 7: The first five frequency parameters $\Omega=\omega R \sqrt{\rho / G}$ of an open isotropic cylindrical shell with various boundary conditions and length-radius ratios $\left(R=1 \mathrm{~m}, h / R=0.3, \theta_{0}=\pi, E=210 \mathrm{GPa}\right.$, and $\left.\mu=0.3\right)$.

\begin{tabular}{|c|c|c|c|c|c|c|c|c|c|c|c|c|}
\hline \multirow{2}{*}{$L / R$} & \multirow{2}{*}{ Mode } & \multicolumn{11}{|c|}{ Boundary conditions } \\
\hline & & FFFF & FFFC & CFFFF & FSFS & FCFC & SFSF & SSSS & SCSC & CFCF & CSCS & CCCC \\
\hline \multirow{5}{*}{1} & 1 & 0.2532 & 0.0611 & 0.6542 & 0.3642 & 0.5435 & 1.0028 & 1.0018 & 1.0018 & 2.2572 & 2.4593 & 2.5851 \\
\hline & 2 & 0.4406 & 0.0894 & 0.7203 & 0.5373 & 0.5644 & 1.4519 & 1.8691 & 1.9529 & 2.2688 & 2.5169 & 2.5995 \\
\hline & 3 & 0.6995 & 0.1871 & 0.9461 & 0.9735 & 0.9928 & 1.4743 & 1.8895 & 2.0027 & 2.4975 & 2.6606 & 2.8954 \\
\hline & 4 & 0.7752 & 0.3095 & 1.1460 & 1.3082 & 1.0391 & 1.8721 & 2.0027 & 2.0362 & 2.5631 & 3.1005 & 3.2424 \\
\hline & 5 & 1.2856 & 0.5914 & 1.4254 & 1.5140 & 1.4765 & 2.0048 & 2.1582 & 2.3938 & 2.9106 & 3.1348 & 3.6945 \\
\hline \multirow{5}{*}{3} & 1 & 0.2028 & 0.0625 & 0.1314 & 0.3720 & 0.5530 & 0.2753 & 0.5713 & 0.8440 & 0.4488 & 0.7125 & 0.9269 \\
\hline & 2 & 0.2595 & 0.1067 & 0.1729 & 0.4243 & 0.7671 & 0.3831 & 0.6442 & 1.0023 & 0.5181 & 0.7552 & 1.1822 \\
\hline & 3 & 0.3976 & 0.1907 & 0.3179 & 0.9237 & 0.8055 & 0.4837 & 1.0023 & 1.1203 & 0.6739 & 1.0461 & 1.5368 \\
\hline & 4 & 0.5753 & 0.2296 & 0.3694 & 0.9898 & 1.0030 & 0.7911 & 1.0461 & 1.4237 & 0.9433 & 1.1792 & 1.5915 \\
\hline & 5 & 0.6844 & 0.4928 & 0.5039 & 1.0467 & 1.1684 & 0.8068 & 1.1140 & 1.4635 & 0.9804 & 1.3171 & 1.7865 \\
\hline \multirow{5}{*}{5} & 1 & 0.1319 & 0.0627 & 0.0669 & 0.3737 & 0.5522 & 0.1314 & 0.3195 & 0.6619 & 0.2043 & 0.4281 & 0.6959 \\
\hline & 2 & 0.2607 & 0.0959 & 0.0682 & 0.3943 & 0.6475 & 0.1778 & 0.4292 & 0.9543 & 0.3012 & 0.4847 & 1.0038 \\
\hline & 3 & 0.2797 & 0.1915 & 0.1718 & 0.5560 & 0.8660 & 0.3288 & 0.6278 & 1.0025 & 0.3742 & 0.6278 & 1.0680 \\
\hline & 4 & 0.3171 & 0.2009 & 0.2425 & 0.6148 & 0.8884 & 0.3638 & 0.6730 & 1.0515 & 0.4729 & 0.7565 & 1.2146 \\
\hline & 5 & 0.3652 & 0.3411 & 0.2779 & 0.6280 & 0.9944 & 0.4033 & 0.7996 & 1.1714 & 0.5441 & 0.8388 & 1.3679 \\
\hline
\end{tabular}



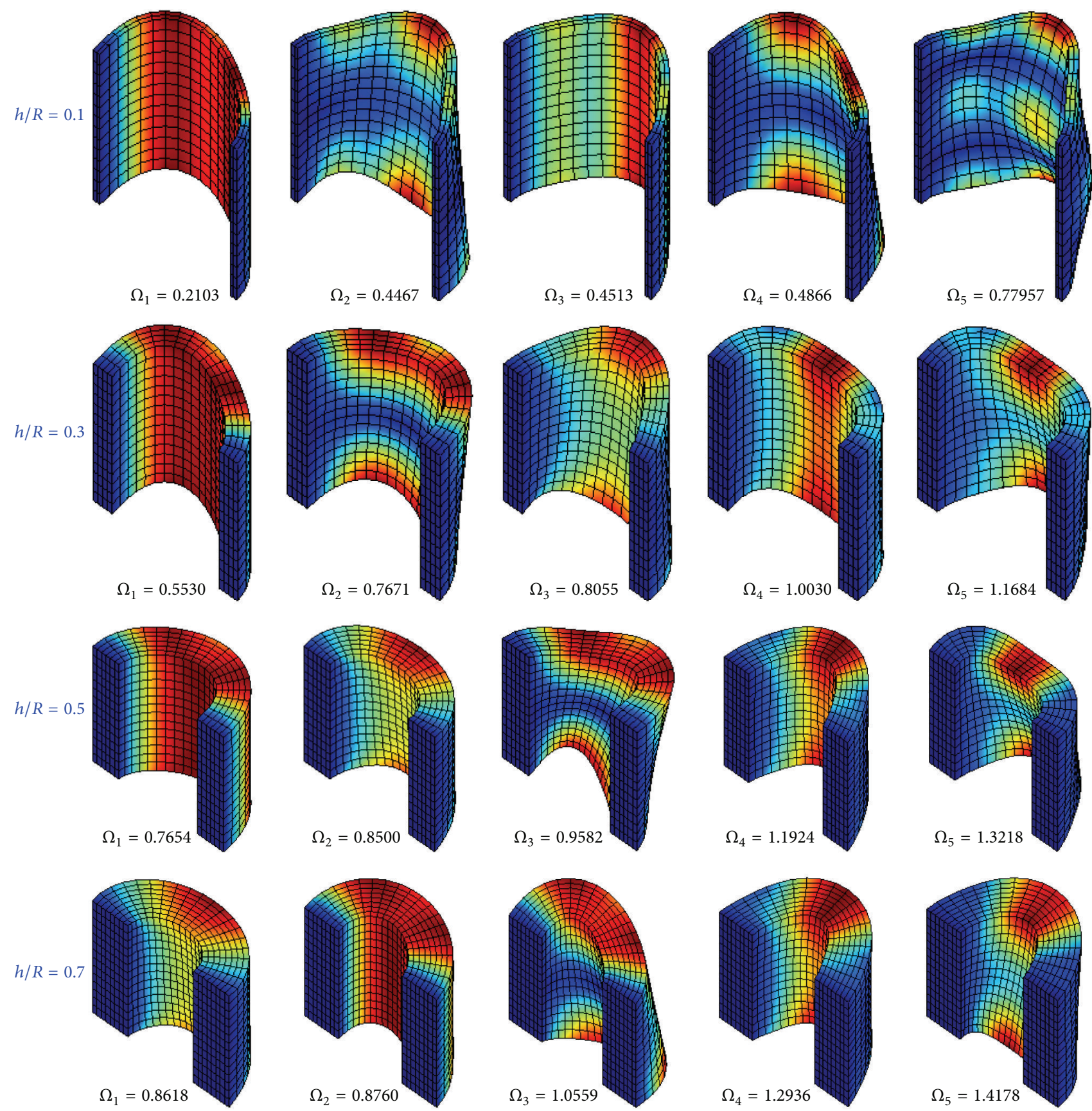

FIGURE 3: Mode shapes of an isotropic cylindrical shell with FCFC boundary conditions and various thickness-radius ratios.

Table 8 shows a comparison made for a thick deep open cylindrical shell $\left(R=1 \mathrm{~m}, L / R=3\right.$, and $\left.\theta_{0}=\pi\right)$ with different sets of boundary conditions. The comparison is performed between the current 3D results and those obtained by ANSYS (SOLID 45, element size: $0.02 \mathrm{~m}$ ). The lowest five frequencies $(\mathrm{Hz})$ are considered in the comparison. From Table 8 , it is obvious that the two results are in good agreement with each other. The proposed three-dimensional improved Fourier series method is applicable for the analysis of orthotropic open cylindrical shells with arbitrary boundary conditions.
Based on the above verification, free vibration results of open cylindrical shells with various boundary conditions and geometric parameters are provided in Tables 9-10 and Figures 5-6. Also, these results can be used to validate new 2D theories and new computational techniques in the future. In Table 9, the first five frequencies are listed for a thick shallow cylindrical panel $\left(R=1 \mathrm{~m}, L / R=2\right.$, and $\left.\theta_{0}=\pi / 2\right)$ with FFFF, FFFC, CFFF, FSFS, FCFC, SFSF, SSSS, SCSC, CFCF, CSCS, and CCCC boundary conditions and different thickness-radius ratios; that is, $h / R=0.1,0.2,0.3,0.4$, and 0.5 . It can be seen from the table that frequencies of the shell 
TABLE 8: Comparison of frequencies $(\mathrm{Hz})$ of an open orthotropic cylindrical shell with different boundary conditions $(L=1 \mathrm{~m}, L / R=3$, $h / R=0.3$, and $\left.\theta_{0}=\pi\right)$.

\begin{tabular}{|c|c|c|c|c|c|c|c|c|c|c|c|}
\hline \multirow{2}{*}{ Method } & \multicolumn{11}{|c|}{ Boundary conditions } \\
\hline & FFFF & FFFC & CFFFF & FSFS & FCFC & SFSF & SSSS & SCSC & CFCF & CSCS & CCCC \\
\hline \multirow{6}{*}{ Present } & 34.030 & 8.8962 & 43.139 & 53.532 & 81.090 & 97.376 & 126.14 & 154.73 & 138.62 & 154.92 & 174.13 \\
\hline & 37.098 & 17.011 & 55.070 & 64.621 & 120.25 & 103.23 & 133.54 & 168.36 & 142.30 & 155.07 & 207.55 \\
\hline & 63.565 & 27.485 & 63.069 & 144.10 & 127.51 & 126.08 & 168.36 & 186.62 & 152.42 & 175.67 & 284.18 \\
\hline & 103.02 & 36.420 & 82.902 & 158.79 & 148.61 & 155.06 & 175.67 & 267.59 & 172.71 & 204.88 & 302.88 \\
\hline & 128.50 & 78.303 & 117.29 & 175.68 & 184.43 & 167.58 & 185.01 & 295.42 & 178.05 & 299.52 & 319.24 \\
\hline & 203.11 & 87.755 & 162.74 & 183.09 & 247.60 & 168.37 & 282.93 & 302.82 & 252.59 & 302.23 & 331.14 \\
\hline \multirow{6}{*}{ ANSYS } & 34.045 & 8.8880 & 43.241 & 53.643 & 80.882 & 97.595 & 126.39 & 154.85 & 139.16 & 155.55 & 174.54 \\
\hline & 37.137 & 16.988 & 55.149 & 64.695 & 120.20 & 103.46 & 133.68 & 168.43 & 142.88 & 155.63 & 208.05 \\
\hline & 63.634 & 27.453 & 63.207 & 144.66 & 127.33 & 126.26 & 168.43 & 186.76 & 152.81 & 175.70 & 284.90 \\
\hline & 103.27 & 36.391 & 82.887 & 159.32 & 148.56 & 155.44 & 175.70 & 267.99 & 172.51 & 205.83 & 303.31 \\
\hline & 128.78 & 78.290 & 117.61 & 175.70 & 184.40 & 167.63 & 185.63 & 295.73 & 178.81 & 301.24 & 320.80 \\
\hline & 203.93 & 87.748 & 163.36 & 183.19 & 247.94 & 168.43 & 284.46 & 304.22 & 253.71 & 304.04 & 332.85 \\
\hline
\end{tabular}
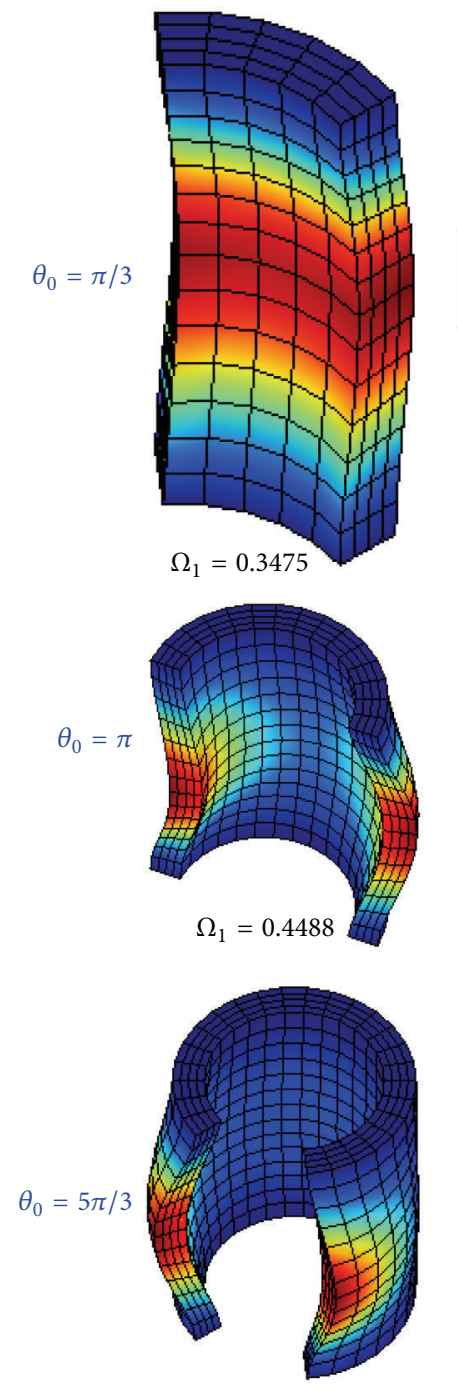

$$
\Omega_{1}=0.4753
$$
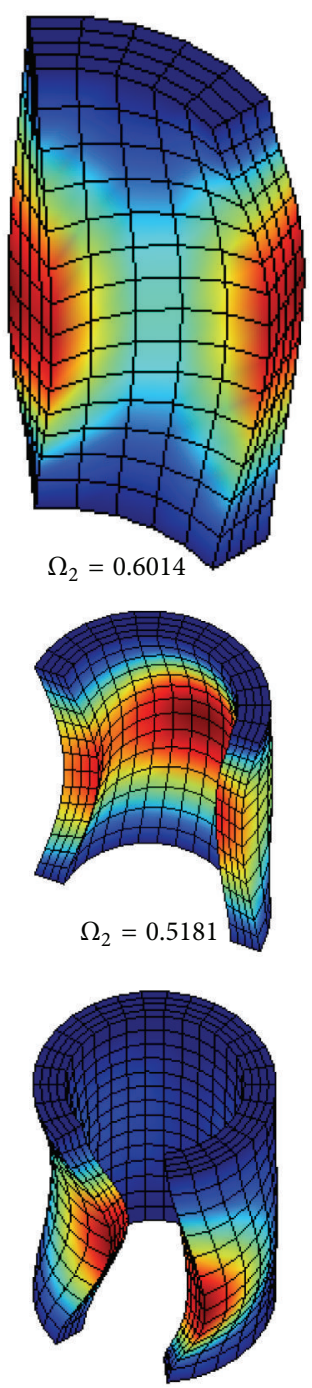

$\Omega_{2}=0.4874$
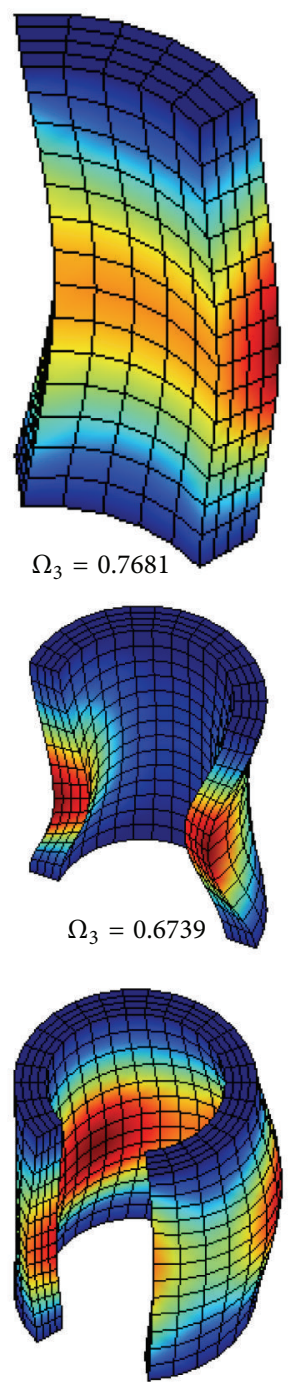

$\Omega_{3}=0.6706$
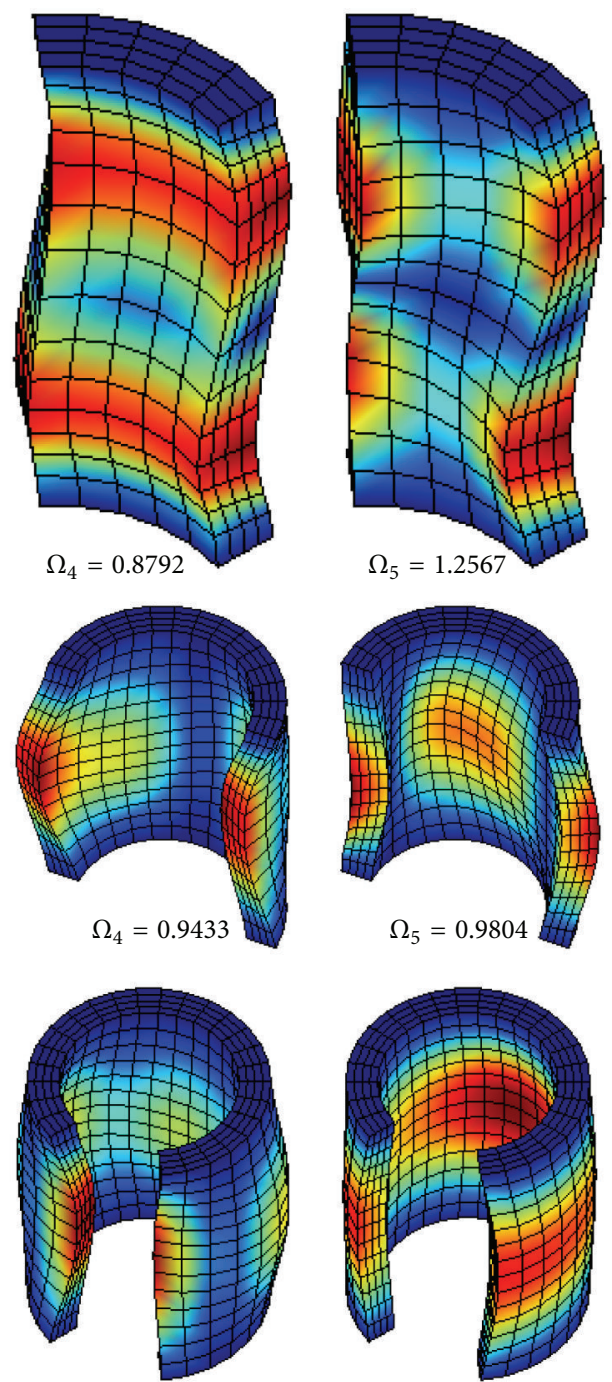

$\Omega_{4}=0.7598$

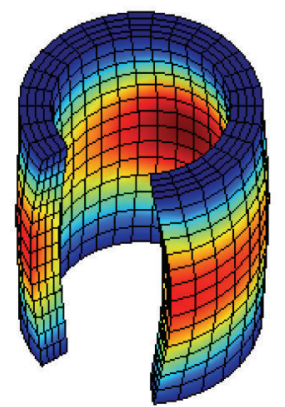

$\Omega_{5}=0.8233$

FIGURE 4: Mode shapes of an isotropic cylindrical shell with CFCF boundary conditions and various circumferential dimensions. 

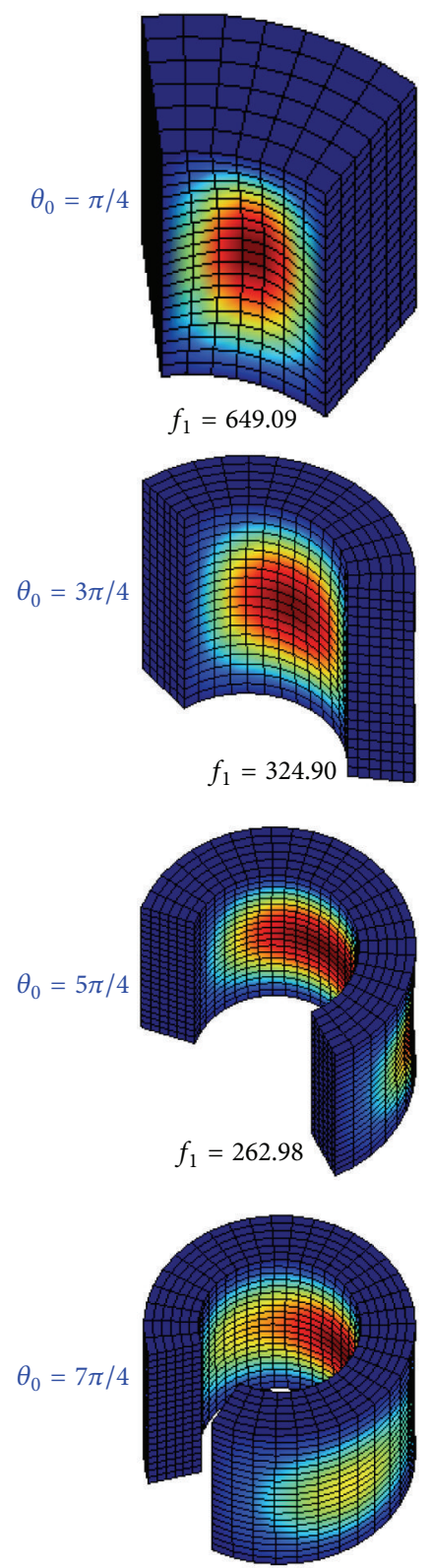

$f_{1}=259.08$
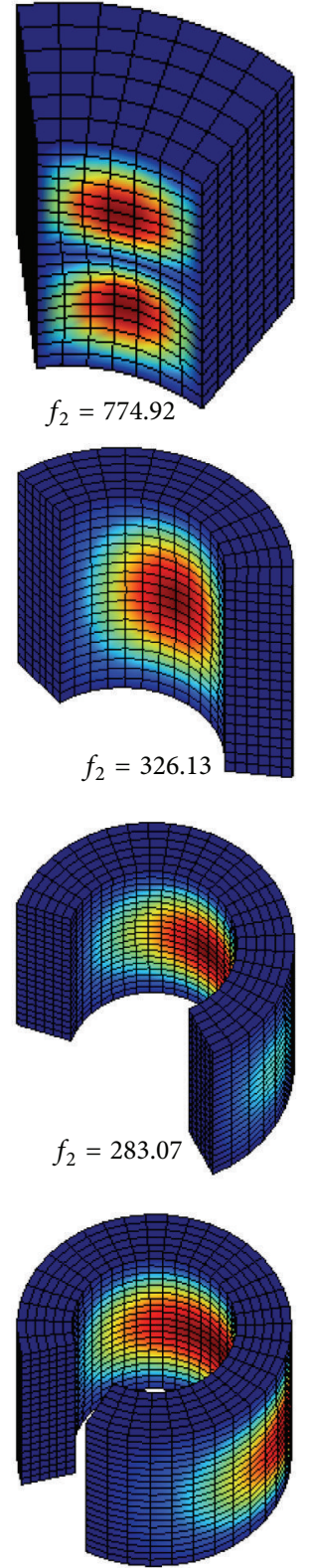

$f_{2}=256.41$
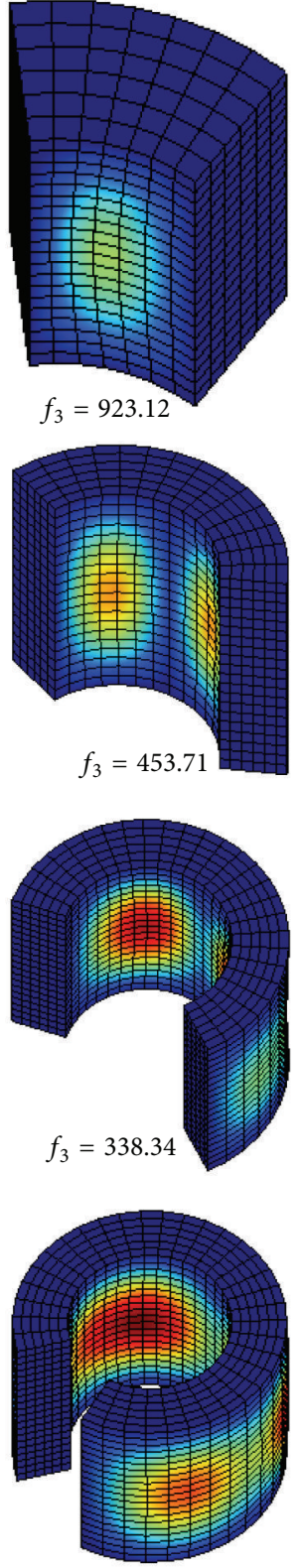

$f_{3}=281.63$
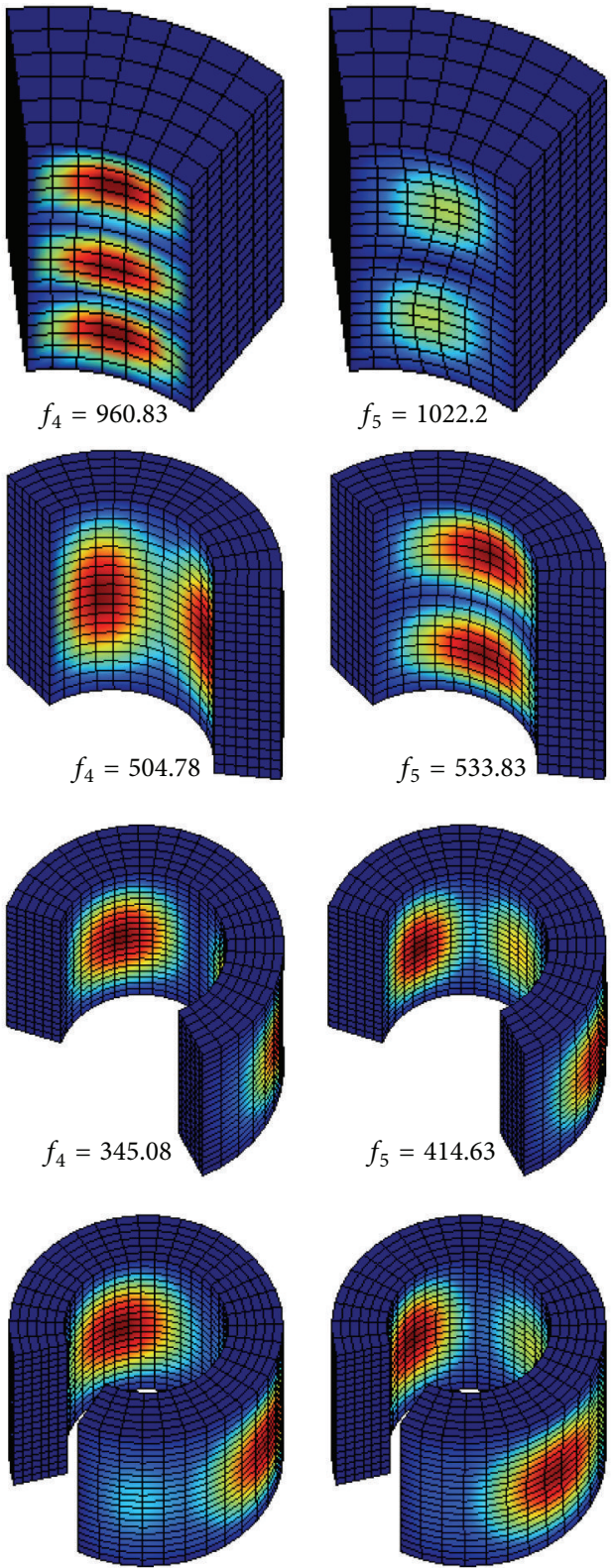

$f_{4}=308.26$

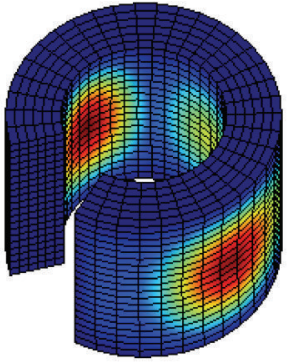

$f_{5}=343.80$

FIGURE 5: Mode shapes of an orthotropic cylindrical shell with CCCC boundary conditions and various circumferential dimensions.

are considerably increased by increasing the thickness-radius ratio. This is due to the fact that, with the increase of the thickness-radius ratio, the shell stiffness increases. Table 10 shows the first five frequencies $(\mathrm{Hz})$ for a rare thick open cylindrical shell subjected to various boundary conditions and circumferential dimensions $\left(\theta_{0}=\pi / 4, \pi / 2,3 \pi / 4, \pi\right.$, and $5 \pi / 4)$. The shell is made from the aforementioned orthotropic materials and with the following geometric parameters: $R=1 \mathrm{~m}, L / R=2$, and $h / R=0.5$. From Table 10 , we can see that the frequencies of the open cylindrical shell reduce by increasing the circumferential dimensions. This is due to the fact that, with the increase of the included angle, the shell stiffness reduces. Also, the lowest five mode shapes for the shell with full clamped boundary conditions are presented in Figure 5 to further enrich the vibration results of orthotropic cylindrical panels. As the last example, variation of the first ten frequencies $(\mathrm{Hz})$ against material parameter $\eta\left(\eta=E_{11} / E_{22}, E_{22}=E_{33}=10 \mathrm{GPa}\right.$, $\mu_{12}=\mu_{13}=0.25, \mu_{23}=0.3$, and $G_{12}=G_{13}=G_{23}=5 \mathrm{GPa}$, $\left.\rho=4500 \mathrm{~kg} / \mathrm{m}^{3}\right)$ for a deep open cylindrical shell $(R=1 \mathrm{~m}$, $L / R=3, h / R=0.3$, and $\theta_{0}=\pi$ ) with FFFF, FFFC, CFFF, CCFF, SSSS, and CCCC is presented in Figure 6. Results of Figure 6 reveal that the effects of the material parameter $\eta$ on the vibration characteristics of open cylindrical shells varied with mode sequences and boundary conditions.

3.4. Open Spherical Shells with Arbitrary Boundary Conditions. In this subsection, the current method is applied 


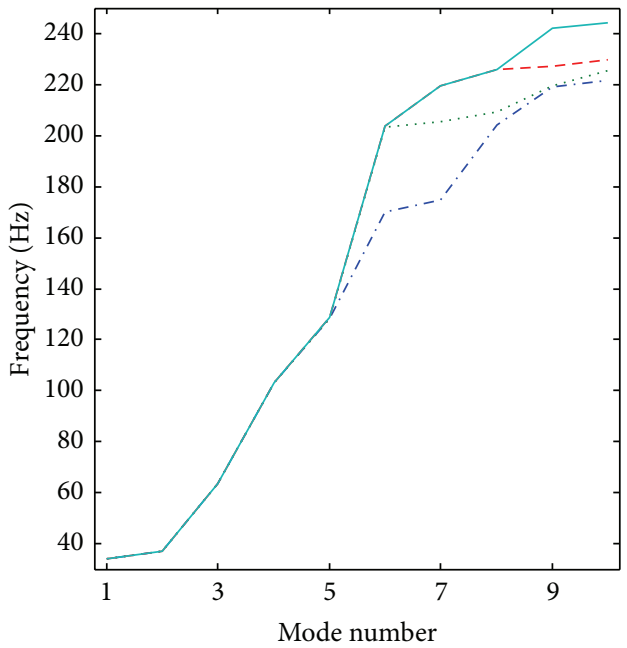

(a) FFFF

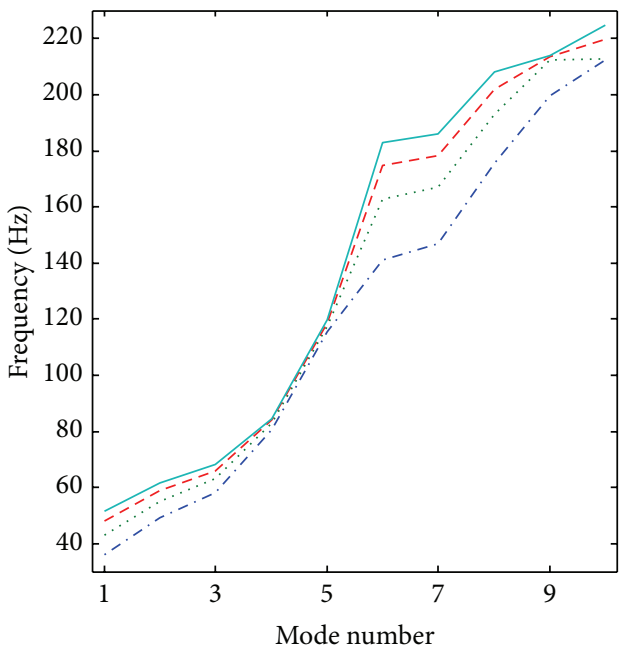

(c) $\mathrm{CFFF}$

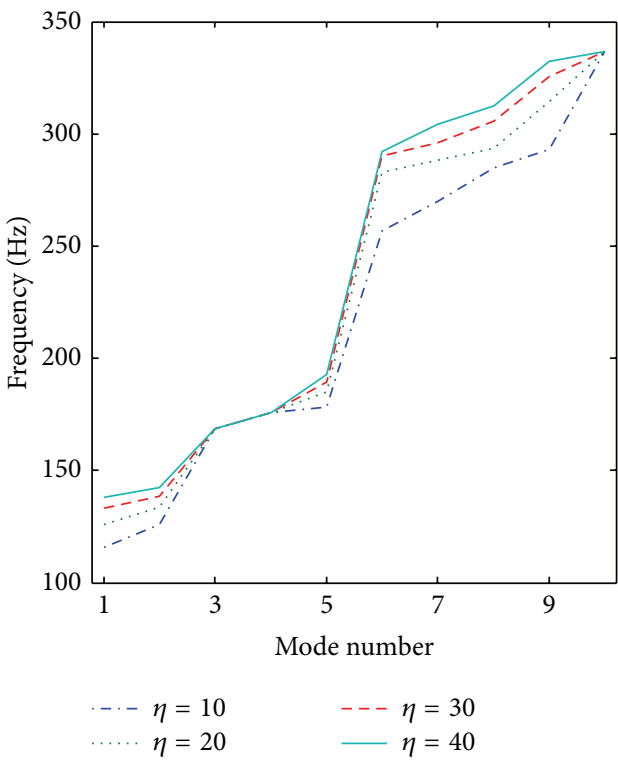

(e) SSSS

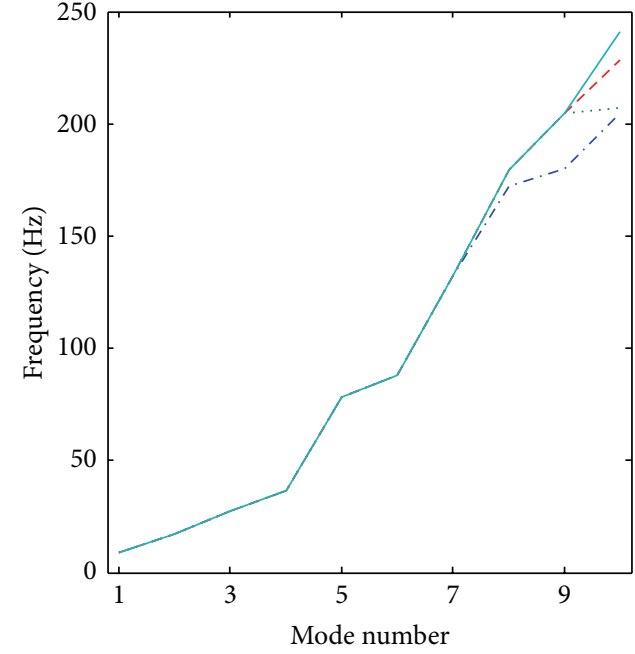

(b) FFFC

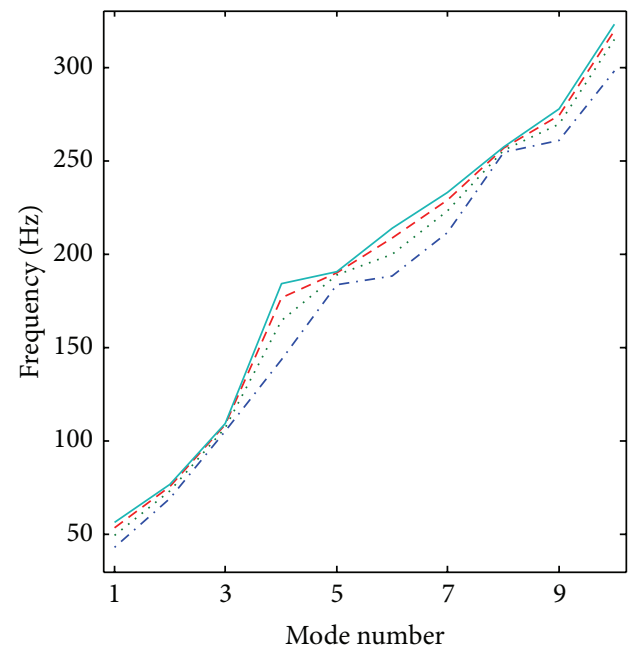

(d) $\mathrm{CCFF}$

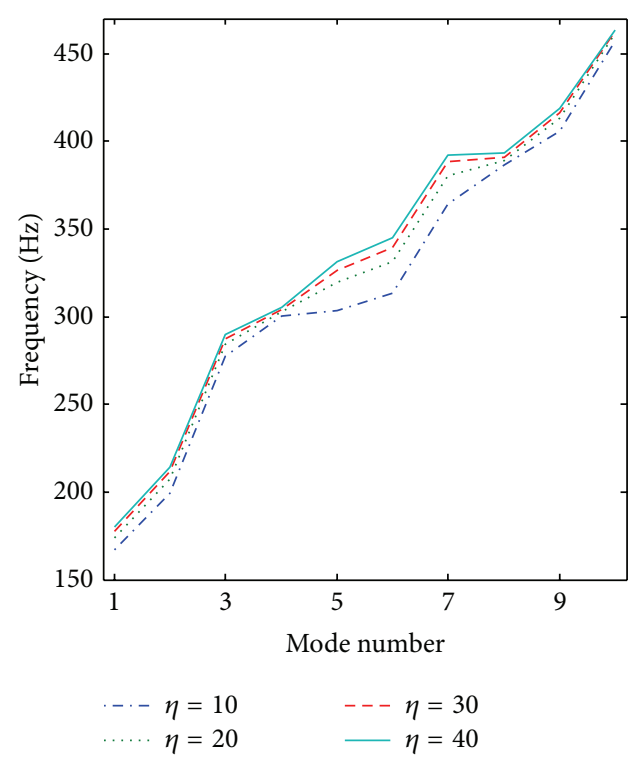

(f) $\mathrm{CCCC}$

FIGURE 6: Variation of the first ten frequencies (Hz) against material parameter $\eta\left(\eta=E_{11} / E_{22}\right)$ for an open cylindrical shell with different boundary conditions. 
TABLE 9: The first five frequencies $(\mathrm{Hz})$ of an open orthotropic cylindrical shell with various boundary conditions and thickness-radius ratios $\left(R=1 \mathrm{~m}, L / R=2\right.$, and $\left.\theta_{0}=\pi / 2\right)$.

\begin{tabular}{|c|c|c|c|c|c|c|c|c|c|c|c|c|}
\hline \multirow{2}{*}{$h / R$} & \multirow{2}{*}{ Mode } & \multicolumn{11}{|c|}{ Boundary conditions } \\
\hline & & FFFF & FFFC & CFFFF & FSFS & FCFC & SFSF & SSSS & SCSC & CFCF & CSCS & CCCC \\
\hline \multirow{5}{*}{0.1} & 1 & 35.215 & 10.253 & 37.165 & 18.324 & 147.58 & 85.29 & 129.33 & 195.62 & 147.58 & 171.95 & 226.11 \\
\hline & 2 & 56.896 & 21.815 & 48.745 & 26.589 & 175.14 & 97.12 & 145.97 & 211.93 & 154.63 & 187.20 & 242.94 \\
\hline & 3 & 84.890 & 48.624 & 77.115 & 97.848 & 190.10 & 127.19 & 255.78 & 334.24 & 172.83 & 263.52 & 361.15 \\
\hline & 4 & 158.64 & 57.907 & 120.79 & 113.01 & 192.42 & 199.13 & 263.52 & 335.67 & 229.70 & 280.45 & 390.23 \\
\hline & 5 & 184.42 & 134.05 & 162.09 & 226.46 & 271.94 & 246.02 & 305.96 & 342.27 & 256.53 & 363.62 & 393.90 \\
\hline \multirow{5}{*}{0.2} & 1 & 67.285 & 20.340 & 61.965 & 36.263 & 220.72 & 140.10 & 168.81 & 263.10 & 203.94 & 218.20 & 301.55 \\
\hline & 2 & 110.39 & 42.002 & 63.152 & 51.258 & 225.46 & 144.40 & 249.83 & 316.59 & 207.32 & 263.52 & 341.24 \\
\hline & 3 & 158.15 & 92.040 & 121.02 & 185.91 & 258.53 & 199.23 & 263.52 & 336.05 & 240.35 & 283.96 & 479.81 \\
\hline & 4 & 291.25 & 95.573 & 136.39 & 211.39 & 289.06 & 246.06 & 336.05 & 447.69 & 256.62 & 441.20 & 520.17 \\
\hline & 5 & 300.44 & 166.90 & 238.77 & 263.52 & 313.52 & 336.05 & 409.13 & 496.74 & 367.82 & 464.15 & 560.42 \\
\hline \multirow{5}{*}{0.3} & 1 & 95.888 & 30.148 & 75.407 & 53.514 & 247.40 & 174.11 & 200.49 & 304.92 & 226.54 & 239.54 & 336.11 \\
\hline & 2 & 158.44 & 60.042 & 80.904 & 73.128 & 252.58 & 177.14 & 263.49 & 336.56 & 226.89 & 263.49 & 408.35 \\
\hline & 3 & 218.13 & 111.91 & 121.17 & 259.77 & 317.94 & 246.32 & 329.58 & 393.01 & 257.12 & 350.80 & 522.17 \\
\hline & 4 & 363.69 & 127.66 & 186.67 & 263.51 & 328.50 & 257.08 & 336.56 & 503.91 & 283.28 & 472.80 & 582.29 \\
\hline & 5 & 370.85 & 195.59 & 272.07 & 290.28 & 361.63 & 336.61 & 454.91 & 574.59 & 458.13 & 526.99 & 585.72 \\
\hline \multirow{5}{*}{0.4} & 1 & 120.95 & 39.497 & 85.560 & 69.868 & 271.91 & 193.37 & 223.05 & 337.19 & 236.27 & 252.31 & 361.36 \\
\hline & 2 & 200.43 & 75.511 & 94.412 & 91.766 & 272.53 & 200.57 & 263.51 & 337.40 & 237.49 & 263.51 & 444.93 \\
\hline & 3 & 266.34 & 119.09 & 121.37 & 263.52 & 325.22 & 246.63 & 337.19 & 434.99 & 257.31 & 403.06 & 549.26 \\
\hline & 4 & 382.10 & 154.90 & 228.50 & 303.73 & 367.10 & 299.86 & 389.76 & 536.97 & 316.14 & 490.52 & 593.38 \\
\hline & 5 & 401.76 & 219.65 & 291.93 & 319.87 & 401.08 & 337.21 & 478.66 & 587.54 & 473.94 & 527.03 & 621.28 \\
\hline \multirow{5}{*}{0.5} & 1 & 142.63 & 48.286 & 93.255 & 85.223 & 283.46 & 205.98 & 239.52 & 337.66 & 241.56 & 261.77 & 381.15 \\
\hline & 2 & 236.61 & 88.344 & 103.65 & 107.20 & 293.00 & 215.57 & 263.52 & 362.31 & 243.65 & 263.52 & 463.94 \\
\hline & 3 & 305.21 & 123.62 & 121.56 & 263.52 & 336.61 & 247.00 & 337.66 & 456.71 & 257.41 & 445.36 & 569.04 \\
\hline & 4 & 382.43 & 175.22 & 263.55 & 309.75 & 387.11 & 333.01 & 436.31 & 559.41 & 343.77 & 502.53 & 600.92 \\
\hline & 5 & 425.37 & 236.77 & 305.95 & 368.50 & 420.72 & 337.67 & 493.40 & 595.89 & 483.80 & 527.04 & 640.08 \\
\hline
\end{tabular}

to study the vibrations of isotropic and orthotropic open spherical shells with arbitrary boundary conditions. As the first example, accuracy of the presented method is validated by comparing the results with solutions published by other researchers as well as those obtained by the FEM analysis. In Table 11, comparison of frequency parameters $\Omega=\omega \sqrt{\rho / E}$ is presented for a FFFF open spherical shell with different thickness-radius ratios. Two different thickness-radius ratios, that is, $h / R=0.05$ and 0.25 , are considered in the comparison. The comparison is carried out between the solutions of Reddy [12], Liew and Lim [13], and Reddy and Liu [14] and the 3D results of Liew et al. [21]. The shell parameters used in the comparison are $R=2 \mathrm{~m}, L_{\phi}=L_{\theta}=4 \sin (0.25)^{-1}, E=$ $210 \mathrm{GPa}$, and $\mu=0.3, \rho=7800 \mathrm{~kg} / \mathrm{m}^{3}$. From the table, we can see that the present results are in good agreement with the $3 \mathrm{D}$ solutions provided by Liew et al. [21]. However, results from different 2D theories deviate significantly as the thicknessradius ratio increases. The comparisons given in Tables 1 and 11 for deep and shallow open spherical shells with FFFF boundary conditions indicate that the current formulation is accurate on the vibration analysis of isotropic open spherical shells with different circumferential dimensions.

Having validated the accuracy of the present method, some further vibration results for isotropic open spherical shells with various boundary conditions, meridional dimensions, thickness-radius ratios, and included angles are presented in Tables 12-14 and Figures 7-9. In Table 12, the first five frequency parameters $\Omega=\omega R \sqrt{\rho\left(1-\mu^{2}\right) / E}$ of an isotropic deep open spherical shell with thickness-radius ratio $h / R=0.1,0.2,0.3,0.4$, and 0.5 are presented. The spherical panel is made from isotropic material $(E=$ $210 \mathrm{GPa}, \mu=0.3$, and $\rho=7800 \mathrm{~kg} / \mathrm{m}^{3}$ ) with top angle $\phi_{0}=\pi / 4$, end angle $\phi_{1}=\pi / 2$, included angle $\theta_{0}=$ $\pi$, and mean radius $R=1 \mathrm{~m}$. Table 12 shows that any increase in the thickness-radius ratio $h / R$, from 0.1 to 0.5 , always leads to corresponding increases in the frequency parameters. Table 13 contains the frequency parameters $\Omega=$ $\omega R \sqrt{\rho\left(1-\mu^{2}\right) / E}$ of an open spherical shell with different boundary conditions and included angles. The shell is made from steel $\left(E=210 \mathrm{GPa}, \mu=0.3\right.$, and $\left.\rho=7800 \mathrm{~kg} / \mathrm{m}^{3}\right)$ with the following geometric parameters: $\phi_{0}=\pi / 4, \phi_{1}=$ $\pi / 2, R=1 \mathrm{~m}$, and $h / R=0.3$. From Table 13, it can be seen that frequency parameters of the shell reduce as the included angle increased. For the sake of completeness, an isotropic deep open spherical shell with different meridional dimensions $\left(\phi_{0}=\right.$ open, $\left.\phi_{1}=5 \pi / 6\right)$ is investigated, and the first five frequency parameters $\Omega=\omega R \sqrt{\rho\left(1-\mu^{2}\right) / E}$ of 
TABLE 10: The first five frequencies $(\mathrm{Hz})$ of an open orthotropic cylindrical shell with various boundary conditions and circumferential dimensions $(R=1 \mathrm{~m}, L / R=2$, and $h / R=0.5)$.

\begin{tabular}{|c|c|c|c|c|c|c|c|c|c|c|c|c|}
\hline \multirow{2}{*}{$\theta_{0}$} & \multirow{2}{*}{ Mode } & \multicolumn{11}{|c|}{ Boundary conditions } \\
\hline & & FFFF & FFFC & CFFFF & FSFS & FCFC & SFSF & SSSS & SCSC & CFCF & CSCS & CCCC \\
\hline \multirow{5}{*}{$\pi / 4$} & 1 & 219.65 & 162.00 & 90.20 & 263.52 & 596.43 & 202.12 & 263.52 & 639.00 & 239.89 & 263.52 & 649.09 \\
\hline & 2 & 419.01 & 208.27 & 108.26 & 368.49 & 601.38 & 228.42 & 436.30 & 666.94 & 248.75 & 445.34 & 774.92 \\
\hline & 3 & 465.14 & 288.48 & 118.35 & 396.92 & 657.70 & 235.54 & 527.03 & 767.71 & 250.46 & 527.03 & 923.12 \\
\hline & 4 & 475.33 & 465.74 & 301.03 & 527.03 & 744.14 & 467.77 & 614.73 & 917.07 & 480.84 & 621.43 & 960.83 \\
\hline & 5 & 597.64 & 468.98 & 334.86 & 594.10 & 886.53 & 489.66 & 666.94 & 953.44 & 498.99 & 790.56 & 1022.2 \\
\hline \multirow{5}{*}{$\pi / 2$} & 1 & 142.63 & 48.286 & 93.255 & 85.223 & 283.46 & 205.98 & 239.52 & 337.66 & 241.56 & 261.77 & 381.15 \\
\hline & 2 & 236.61 & 88.344 & 103.65 & 107.20 & 293.00 & 215.57 & 263.52 & 362.31 & 243.65 & 263.52 & 463.94 \\
\hline & 3 & 305.21 & 123.62 & 121.56 & 263.52 & 336.61 & 247.00 & 337.66 & 456.71 & 257.41 & 445.36 & 569.04 \\
\hline & 4 & 382.43 & 175.22 & 263.55 & 309.75 & 387.11 & 333.01 & 436.31 & 559.41 & 343.77 & 502.53 & 600.92 \\
\hline & 5 & 425.37 & 236.77 & 305.95 & 368.50 & 420.72 & 337.67 & 493.40 & 595.89 & 483.80 & 527.04 & 640.08 \\
\hline \multirow{5}{*}{$3 \pi / 4$} & 1 & 99.221 & 23.555 & 98.027 & 20.826 & 179.51 & 209.92 & 225.65 & 225.65 & 242.42 & 250.22 & 324.90 \\
\hline & 2 & 109.37 & 44.625 & 99.777 & 29.006 & 202.85 & 213.14 & 227.82 & 303.60 & 244.87 & 263.52 & 326.13 \\
\hline & 3 & 176.95 & 80.391 & 125.40 & 169.39 & 216.45 & 225.65 & 263.52 & 313.24 & 259.75 & 300.22 & 453.71 \\
\hline & 4 & 272.57 & 80.724 & 153.80 & 197.59 & 249.77 & 249.05 & 282.55 & 447.35 & 269.40 & 445.40 & 504.78 \\
\hline & 5 & 302.46 & 147.74 & 295.33 & 232.97 & 251.32 & 252.85 & 436.36 & 448.72 & 369.96 & 454.29 & 533.83 \\
\hline \multirow{5}{*}{$\pi$} & 1 & 60.064 & 14.609 & 97.256 & 85.225 & 115.29 & 169.38 & 169.38 & 169.38 & 242.90 & 251.99 & 278.41 \\
\hline & 2 & 72.226 & 25.387 & 106.05 & 107.22 & 122.85 & 209.72 & 232.59 & 263.60 & 247.89 & 261.78 & 303.32 \\
\hline & 3 & 121.09 & 43.529 & 119.40 & 207.90 & 172.43 & 216.88 & 239.54 & 280.92 & 252.31 & 263.52 & 383.96 \\
\hline & 4 & 158.40 & 58.760 & 127.22 & 216.26 & 177.78 & 229.93 & 263.52 & 337.66 & 260.93 & 329.36 & 388.97 \\
\hline & 5 & 213.58 & 104.54 & 193.31 & 245.57 & 218.35 & 255.73 & 314.27 & 372.97 & 294.36 & 410.43 & 503.93 \\
\hline \multirow{5}{*}{$5 \pi / 4$} & 1 & 37.256 & 10.494 & 98.891 & 7.6304 & 68.855 & 135.56 & 135.56 & 135.56 & 244.19 & 251.98 & 262.98 \\
\hline & 2 & 53.428 & 16.689 & 105.17 & 11.087 & 86.167 & 211.63 & 228.82 & 246.85 & 246.03 & 254.46 & 283.07 \\
\hline & 3 & 91.135 & 26.660 & 113.22 & 43.505 & 130.86 & 213.79 & 238.09 & 262.43 & 252.19 & 263.52 & 338.34 \\
\hline & 4 & 98.780 & 42.932 & 128.30 & 58.120 & 136.00 & 232.15 & 261.83 & 270.55 & 261.63 & 281.55 & 345.08 \\
\hline & 5 & 153.66 & 78.062 & 145.98 & 133.96 & 189.18 & 244.79 & 263.52 & 323.93 & 265.85 & 349.53 & 414.63 \\
\hline
\end{tabular}

TABLE 11: Comparison of frequency parameters $\Omega=\omega \sqrt{\rho / E}$ of a FFFF open isotropic spherical shell with different thickness-radius ratios $\left(R=2 \mathrm{~m}, L_{\phi}=L_{\theta}=4 \sin (0.25)^{-1} \mathrm{~m}, E=210 \mathrm{GPa}, \mu=0.3\right.$, and $\left.\rho=7800 \mathrm{~kg} / \mathrm{m}^{3}\right)$.

\begin{tabular}{|c|c|c|c|c|c|c|c|c|c|c|}
\hline \multirow{2}{*}{$h / R$} & \multirow{2}{*}{ Theory } & \multicolumn{9}{|c|}{ Mode number } \\
\hline & & SS-1 & SS-2 & SS-3 & SA-1 & SA-2 & SA-3 & AA-1 & AA-2 & AA-3 \\
\hline \multirow{4}{*}{0.05} & FSDT [13] & 0.57042 & 0.78415 & 1.7244 & 0.96545 & 1.7084 & 2.6277 & 0.38434 & 1.8309 & 2.0684 \\
\hline & FSDT [14] & 0.56635 & 0.77992 & 1.7210 & 0.96218 & 1.7025 & 2.6283 & 0.38299 & 1.8272 & 2.0631 \\
\hline & $3-\mathrm{D}[21]$ & 0.56477 & 0.76885 & 1.7153 & 0.97260 & 1.6719 & 2.6202 & 0.38566 & 1.8324 & 2.0605 \\
\hline & Present & 0.56479 & 0.76890 & 1.7163 & 0.97300 & 1.6720 & 2.6202 & 0.38581 & 1.8330 & 2.0610 \\
\hline \multirow{5}{*}{0.25} & FSDT [13] & 1.8691 & 2.2768 & 2.7555 & 2.5575 & 2.6627 & 3.4526 & 1.3089 & 2.4434 & 3.2452 \\
\hline & TSDT [14] & 1.8689 & 2.2706 & 2.7499 & 2.5500 & 2.7059 & 3.4492 & 1.3216 & 2.4367 & 3.2554 \\
\hline & HSDT [12] & 1.8759 & 2.2875 & 2.7524 & 2.5545 & 2.6794 & 3.4701 & 1.3142 & 2.4441 & 3.2577 \\
\hline & $3-\mathrm{D}[21]$ & 1.8665 & 2.2390 & 2.7317 & 2.5242 & 2.6792 & 3.4627 & 1.3191 & 2.4199 & 3.2979 \\
\hline & Present & 1.8641 & 2.2347 & 2.7315 & 2.5232 & 2.6738 & 3.4691 & 1.3176 & 2.4198 & 3.2945 \\
\hline
\end{tabular}

the shell are given in Table 14. The material and geometric properties used in the investigation are $\theta_{0}=\pi, R=1 \mathrm{~m}$, $h / R=0.3, E=210 \mathrm{GPa}$, and $\mu=0.3$. In order to enhance our understanding of deep open spherical shell with different meridional dimensions, some selected mode shapes for the shell with CFCF boundary conditions are presented in Figure 7. Because of the curvature of the middle surface, the mode shapes for the doubly curved spherical shells are much more complicated than the singly curved cylindrical ones.
Then, free vibration analyses of deep orthotropic spherical shells with arbitrary boundary conditions are considered in the following discussions, for which very limited amount of results is available in the literature. In Table 15, the lowest five frequencies $(\mathrm{Hz})$ of an orthotropic deep open spherical shell with eleven combinations of boundary conditions are presented for five different thickness-radius ratios; that is, $h / R=0.1,0.2,0.3,0.4$, and 0.5 . The other geometrical parameters used for the analysis are $\phi_{0}=\pi / 4, \phi_{1}=\pi / 2$, 
TABLE 12: The first five frequency parameters $\Omega=\omega R \sqrt{\rho\left(1-\mu^{2}\right) / E}$ of an open isotropic spherical shell with various boundary conditions and thickness-radius ratios $\left(\phi_{0}=\pi / 4, \phi_{1}=\pi / 2, \theta_{0}=\pi, R=1 \mathrm{~m}, E=210 \mathrm{GPa}\right.$, and $\left.\mu=0.3\right)$.

\begin{tabular}{|c|c|c|c|c|c|c|c|c|c|c|c|c|}
\hline \multirow{2}{*}{$h / R$} & \multirow{2}{*}{ Mode } & \multicolumn{11}{|c|}{ Boundary conditions } \\
\hline & & FFFF & FFFC & CFFFF & FSFS & FCFC & SFSF & SSSS & SCSC & CFCF & CSCS & CCCC \\
\hline \multirow{5}{*}{0.1} & 1 & 0.0711 & 0.0172 & 0.2269 & 0.0951 & 0.1658 & 0.2564 & 0.6355 & 0.6421 & 1.2226 & 1.4486 & 1.4869 \\
\hline & 2 & 0.1353 & 0.0254 & 0.2549 & 0.2163 & 0.2382 & 0.4922 & 1.0055 & 1.0248 & 1.2226 & 1.4581 & 1.4937 \\
\hline & 3 & 0.2032 & 0.0561 & 0.3066 & 0.2628 & 0.3729 & 0.5117 & 1.1114 & 1.1206 & 1.4630 & 1.5212 & 1.6187 \\
\hline & 4 & 0.2812 & 0.1111 & 0.4167 & 0.4866 & 0.5115 & 0.6871 & 1.1169 & 1.1836 & 1.4899 & 1.5563 & 1.6193 \\
\hline & 5 & 0.3964 & 0.1835 & 0.4735 & 0.5418 & 0.6370 & 1.0485 & 1.2577 & 1.3111 & 1.5498 & 1.6713 & 1.7849 \\
\hline \multirow{5}{*}{0.2} & 1 & 0.1316 & 0.0314 & 0.3703 & 0.1748 & 0.2876 & 0.3148 & 0.6914 & 0.7021 & 1.7007 & 1.8791 & 1.9375 \\
\hline & 2 & 0.2489 & 0.0458 & 0.3938 & 0.3473 & 0.3054 & 0.6826 & 1.2074 & 1.2261 & 1.7017 & 1.9277 & 1.9540 \\
\hline & 3 & 0.3663 & 0.1008 & 0.5050 & 0.4708 & 0.5767 & 0.8509 & 1.2620 & 1.2844 & 1.9172 & 1.9462 & 2.0873 \\
\hline & 4 & 0.4801 & 0.1765 & 0.5452 & 0.8508 & 0.6582 & 0.8631 & 1.4002 & 1.4558 & 1.9183 & 2.1248 & 2.2129 \\
\hline & 5 & 0.6947 & 0.3174 & 0.8061 & 0.8706 & 0.9080 & 1.2096 & 1.4917 & 1.5681 & 2.0520 & 2.2411 & 2.5283 \\
\hline \multirow{5}{*}{0.3} & 1 & 0.1888 & 0.0452 & 0.4689 & 0.2480 & 0.3235 & 0.3414 & 0.7145 & 0.7274 & 1.9243 & 2.1076 & 2.1555 \\
\hline & 2 & 0.3385 & 0.0611 & 0.5233 & 0.4129 & 0.3853 & 0.6941 & 1.2672 & 1.2855 & 1.9255 & 2.1111 & 2.1944 \\
\hline & 3 & 0.5085 & 0.1409 & 0.5676 & 0.6466 & 0.6937 & 1.0925 & 1.4292 & 1.4529 & 2.1144 & 2.2186 & 2.3711 \\
\hline & 4 & 0.5841 & 0.2079 & 0.7232 & 0.9648 & 0.6970 & 1.1003 & 1.5709 & 1.6321 & 2.1613 & 2.2262 & 2.4572 \\
\hline & 5 & 0.9281 & 0.4222 & 0.8582 & 1.0133 & 0.9779 & 1.2698 & 1.7908 & 1.8377 & 2.2045 & 2.4715 & 2.6920 \\
\hline \multirow{5}{*}{0.4} & 1 & 0.2416 & 0.0581 & 0.5509 & 0.3124 & 0.3313 & 0.3516 & 0.7190 & 0.7330 & 2.0016 & 2.1819 & 2.2376 \\
\hline & 2 & 0.4045 & 0.0721 & 0.6014 & 0.4431 & 0.4574 & 0.6900 & 1.2629 & 1.2838 & 2.0067 & 2.1821 & 2.3231 \\
\hline & 3 & 0.6196 & 0.1755 & 0.6434 & 0.7852 & 0.7009 & 1.2435 & 1.5776 & 1.6009 & 2.1864 & 2.2299 & 2.5276 \\
\hline & 4 & 0.6282 & 0.2233 & 0.8223 & 0.9835 & 0.7571 & 1.2719 & 1.7479 & 1.8035 & 2.2195 & 2.3895 & 2.5360 \\
\hline & 5 & 0.9931 & 0.4734 & 0.8837 & 1.0620 & 0.9996 & 1.2827 & 1.8404 & 1.8563 & 2.3305 & 2.6176 & 2.8487 \\
\hline \multirow{5}{*}{0.5} & 1 & 0.2892 & 0.0699 & 0.6158 & 0.3652 & 0.3349 & 0.3535 & 0.7139 & 0.7283 & 1.9969 & 2.1339 & 2.2429 \\
\hline & 2 & 0.4499 & 0.0801 & 0.6460 & 0.4582 & 0.5071 & 0.6805 & 1.2441 & 1.2659 & 2.0163 & 2.1928 & 2.3929 \\
\hline & 3 & 0.6208 & 0.2042 & 0.7185 & 0.8844 & 0.6967 & 1.2397 & 1.6955 & 1.7173 & 2.1961 & 2.2960 & 2.5692 \\
\hline & 4 & 0.7268 & 0.2311 & 0.8231 & 0.9934 & 0.7913 & 1.3794 & 1.8064 & 1.8236 & 2.2815 & 2.4889 & 2.6311 \\
\hline & 5 & 0.9992 & 0.4899 & 0.9674 & 1.0810 & 1.0059 & 1.3841 & 1.8848 & 1.9327 & 2.4229 & 2.6261 & 2.9453 \\
\hline
\end{tabular}

$R=1 \mathrm{~m}$, and $\theta_{0}=\pi$. In order to verify the accuracy of the present work, corresponding frequencies obtained by the FE analysis (ANSYS, SOLID 45, element size: $0.02 \mathrm{~m}$ ) of the shell with thickness-radius ratio $h / R=0.3$ are included in the table as well. From Table 15, one can observe that the present results are in good agreement with the $3 \mathrm{D}$ elasticity solutions obtained by the FE analysis. The table also reveals that the frequency parameters of the shell increase with the thicknessradius ratio increases. Then, the first five frequencies for an open orthotropic spherical shell $\left(\phi_{0}=\pi / 4, \phi_{1}=\pi / 2, R=\right.$ $1 \mathrm{~m}$, and $h / R=0.3$ ) involving different boundary conditions and circumferential dimensions are given in Table 16. As expected, frequencies of the spherical shell vary from the maximum value for the $\theta_{0}=\pi / 4$ shell to the minimum value for the $\theta_{0}=5 \pi / 4$ one. In Table 17 , frequencies of an open orthotropic spherical shell with various boundary conditions and meridional dimensions are presented. The shell with top angle $\phi_{0}=2 \pi / 3, \pi / 2, \pi / 3$, or $\pi / 6$, end angle $\phi_{1}=5 \pi / 6$, included angle $\theta_{0}=\pi$, mean radius $R=$ $1 \mathrm{~m}$, and thickness-radius ratio $h / R=0.3$ is considered in the analysis. Unlike cylindrical shells, with increasing the meridional dimensions of a spherical shell, the stiffness of the shell may not reduce. The vibration characteristics of thick open orthotropic spherical shells can be further elaborated by
Figures 8-9 by considering the mode shapes of open spherical shells with FCFC boundary conditions and CCCC boundary conditions as their frequencies presented in Tables 15 and 16, respectively. Finally, variation of the first ten frequencies $(\mathrm{Hz})$ against material parameter $\eta\left(\eta=E_{11} / E_{22}, E_{22}=E_{33}=\right.$ $10 \mathrm{GPa}, \mu_{12}=\mu_{13}=0.25, \mu_{23}=0.3, G_{12}=G_{13}=G_{23}=$ $5 \mathrm{GPa}$, and $\rho=4500 \mathrm{~kg} / \mathrm{m}^{3}$ ) of a deep open spherical shell $\left(\phi_{0}=\pi / 4, \phi_{1}=\pi / 2, R=1 \mathrm{~m}\right.$, and $\left.h / R=0.3, \theta_{0}=\pi\right)$ with FFFF, FFFC, CFFF, CCFF, SSSS, and CCCC is presented in Figure 10. By comparing Figures 10 and 6, we can see that the effects of the material parameter $\eta$ on the vibration characteristics of open shells varied with mode sequences, boundary conditions, and shell types.

\section{Conclusions}

This paper presents a unified method for the free vibration analysis of isotropic and orthotropic plates and shells with arbitrary boundary conditions and geometry dimensions in the framework of three-dimensional displacement-based energy formulation, including rectangular plates, open cylindrical shells, and the spherical ones. Regardless of boundary conditions, displacement in each structure direction is expanded as a triplicate product of the cosine Fourier 
TABLE 13: The first five frequency parameters $\Omega=\omega R \sqrt{\rho\left(1-\mu^{2}\right) / E}$ of an open isotropic spherical shell with various boundary conditions and included angles $\left(\phi_{0}=\pi / 4, \phi_{1}=\pi / 2, R=1 \mathrm{~m}, h / R=0.3, E=210 \mathrm{GPa}\right.$, and $\left.\mu=0.3\right)$.

\begin{tabular}{|c|c|c|c|c|c|c|c|c|c|c|c|c|}
\hline \multirow{2}{*}{$\theta_{0}$} & \multirow{2}{*}{ Mode } & \multicolumn{11}{|c|}{ Boundary conditions } \\
\hline & & FFFF & FFFC & CFFFF & FSFS & FCFC & SFSF & SSSS & SCSC & CFCF & CSCS & CCCC \\
\hline \multirow{5}{*}{$\pi / 4$} & 1 & 1.5434 & 0.5258 & 0.3956 & 1.1290 & 1.9941 & 0.2223 & 2.1807 & 2.4911 & 1.8990 & 2.2182 & 3.0667 \\
\hline & 2 & 2.0638 & 0.8659 & 0.5747 & 1.6591 & 2.4592 & 1.0025 & 2.2182 & 2.7231 & 1.9102 & 2.4706 & 4.1467 \\
\hline & 3 & 2.5408 & 1.0483 & 0.7678 & 2.2636 & 2.5999 & 1.2964 & 2.4712 & 4.1293 & 2.2036 & 3.9360 & 4.3251 \\
\hline & 4 & 2.9590 & 1.8398 & 1.5304 & 2.4774 & 3.4859 & 1.9852 & 3.6766 & 4.1563 & 3.3344 & 4.3027 & 4.7985 \\
\hline & 5 & 3.1493 & 2.2736 & 1.9231 & 3.2833 & 3.5635 & 2.5928 & 4.0103 & 4.5780 & 3.4008 & 4.3531 & 4.9817 \\
\hline \multirow{5}{*}{$\pi / 2$} & 1 & 0.7468 & 0.1503 & 0.4323 & 0.2479 & 0.8889 & 0.2957 & 1.2670 & 1.3054 & 1.9184 & 2.1075 & 2.3646 \\
\hline & 2 & 0.8246 & 0.2622 & 0.5281 & 0.4128 & 1.2374 & 1.0485 & 1.5707 & 1.7150 & 1.9212 & 2.2185 & 2.5819 \\
\hline & 3 & 1.3824 & 0.5249 & 0.7754 & 1.1291 & 1.3836 & 1.1472 & 2.1811 & 2.3482 & 2.1685 & 2.4709 & 3.2073 \\
\hline & 4 & 1.7188 & 0.6080 & 0.9511 & 1.2559 & 1.4004 & 1.3091 & 2.2185 & 2.4407 & 2.2848 & 3.1325 & 3.4764 \\
\hline & 5 & 1.8750 & 1.0441 & 1.5353 & 1.6594 & 1.8157 & 1.9292 & 2.4717 & 3.0104 & 2.8882 & 3.2707 & 3.5592 \\
\hline \multirow{5}{*}{$3 \pi / 4$} & 1 & 0.3418 & 0.0727 & 0.4725 & 0.0601 & 0.5198 & 0.3268 & 0.8917 & 0.9135 & 1.9210 & 2.0967 & 2.2406 \\
\hline & 2 & 0.5192 & 0.1124 & 0.4959 & 0.0979 & 0.6987 & 0.8800 & 1.4624 & 1.5154 & 1.9236 & 2.1724 & 2.2484 \\
\hline & 3 & 0.8172 & 0.2652 & 0.6403 & 0.5017 & 0.9143 & 1.0832 & 1.6389 & 1.6688 & 2.1366 & 2.2186 & 2.6379 \\
\hline & 4 & 0.8719 & 0.3327 & 0.8189 & 0.8047 & 1.0622 & 1.1156 & 1.7514 & 1.8452 & 2.1756 & 2.4713 & 2.7068 \\
\hline & 5 & 1.3417 & 0.6414 & 1.0216 & 1.0307 & 1.0907 & 1.5621 & 2.1816 & 2.3033 & 2.3414 & 2.7596 & 3.1727 \\
\hline \multirow{5}{*}{$\pi$} & 1 & 0.1888 & 0.0452 & 0.4689 & 0.2480 & 0.3235 & 0.3414 & 0.7145 & 0.7274 & 1.9243 & 2.1076 & 2.1555 \\
\hline & 2 & 0.3385 & 0.0611 & 0.5233 & 0.4129 & 0.3853 & 0.6941 & 1.2672 & 1.2855 & 1.9255 & 2.1111 & 2.1944 \\
\hline & 3 & 0.5085 & 0.1409 & 0.5676 & 0.6466 & 0.6937 & 1.0925 & 1.4292 & 1.4529 & 2.1144 & 2.2186 & 2.3711 \\
\hline & 4 & 0.5841 & 0.2079 & 0.7232 & 0.9648 & 0.6970 & 1.1003 & 1.5709 & 1.6321 & 2.1613 & 2.2262 & 2.4572 \\
\hline & 5 & 0.9281 & 0.4222 & 0.8582 & 1.0133 & 0.9779 & 1.2698 & 1.7908 & 1.8377 & 2.2045 & 2.4715 & 2.6920 \\
\hline \multirow{5}{*}{$5 \pi / 4$} & 1 & 0.1177 & 0.0327 & 0.4867 & 0.0224 & 0.2086 & 0.3497 & 0.6155 & 0.6236 & 1.9278 & 2.0948 & 2.1323 \\
\hline & 2 & 0.2184 & 0.0398 & 0.4900 & 0.0319 & 0.2259 & 0.5910 & 1.0399 & 1.0542 & 1.9282 & 2.1264 & 2.1506 \\
\hline & 3 & 0.3231 & 0.0859 & 0.6039 & 0.1257 & 0.4760 & 1.0334 & 1.4150 & 1.4271 & 2.1169 & 2.1401 & 2.2870 \\
\hline & 4 & 0.4395 & 0.1313 & 0.6105 & 0.2094 & 0.4815 & 1.0978 & 1.4948 & 1.5098 & 2.1215 & 2.2187 & 2.2974 \\
\hline & 5 & 0.6178 & 0.2576 & 0.8022 & 0.3940 & 0.7802 & 1.1040 & 1.4989 & 1.5356 & 2.1970 & 2.2654 & 2.5086 \\
\hline
\end{tabular}

series with the addition of certain supplementary terms introduced to eliminate any possible jumps with the original displacement function and its relevant derivatives at the boundaries. The boundary conditions of the shells and plates are accounted for by using the artificial spring technique and any classical boundary conditions can be easily realized by assigning the springs with corresponding values, without the need of making any modifications to the trial functions and solution program to adapt to different boundary cases. A unified computer code based on the scientific software MATLAB is developed to obtain the accurate $3 \mathrm{D}$ vibration results by using the Rayleigh-Ritz procedure. The validation of the current method, theoretical formulations, and program code is proved by comparing results with solutions in the open literature as well as data by the $3 \mathrm{D} \mathrm{FE}$ analysis. It is seen that the current results are very close to the referential ones. The effects of boundary conditions, material parameters, and geometric dimensions on the frequencies are discussed as well. Finally, several $3 \mathrm{D}$ vibration results of isotropic and orthotropic plates and open cylindrical and spherical shells with different geometry dimensions are presented for various boundary conditions, which may serve as benchmark solutions for future researches as well as structure designers in this field.

\section{Appendices}

\section{A. Strain-Displacement Relations for the Plates and Cylindrical and Spherical Shells}

Based on the rectangular coordinate system given in Figure 1(b), the strain-displacement relations for plates are

$$
\begin{aligned}
\varepsilon_{x} & =\frac{\partial u}{\partial x} \\
\varepsilon_{y} & =\frac{\partial v}{\partial y}, \\
\varepsilon_{z} & =\frac{\partial w}{\partial z}, \\
\gamma_{x y} & =\frac{\partial v}{\partial x}+\frac{\partial u}{\partial y},
\end{aligned}
$$


TABLE 14: The first five frequency parameters $\Omega=\omega R \sqrt{\rho\left(1-\mu^{2}\right) / E}$ of an open isotropic spherical shell with various boundary conditions and meridian dimensions $\left(\phi_{1}=5 \pi / 6, \theta_{0}=\pi, R=1 \mathrm{~m}, h / R=0.3, E=210 \mathrm{GPa}\right.$, and $\left.\mu=0.3\right)$.

\begin{tabular}{|c|c|c|c|c|c|c|c|c|c|c|c|c|}
\hline \multirow{2}{*}{$\phi_{0}$} & \multirow{2}{*}{ Mode } & \multicolumn{11}{|c|}{ Boundary conditions } \\
\hline & & FFFF & FFFC & CFFFF & FSFS & FCFC & SFSF & SSSS & SCSC & CFCF & CSCS & CCCC \\
\hline \multirow{5}{*}{$2 \pi / 3$} & 1 & 0.3396 & 0.0736 & 1.2127 & 0.3772 & 0.3464 & 0.7931 & 1.1614 & 1.1952 & 3.0624 & 3.2486 & 3.3176 \\
\hline & 2 & 0.5312 & 0.0951 & 1.2320 & 0.5875 & 0.5971 & 1.0303 & 1.7034 & 1.7680 & 3.0640 & 3.2887 & 3.3482 \\
\hline & 3 & 0.7473 & 0.2249 & 1.5289 & 0.9444 & 0.8254 & 1.6863 & 2.3589 & 2.3823 & 3.2730 & 3.4337 & 3.5946 \\
\hline & 4 & 0.8582 & 0.2766 & 1.9177 & 1.2117 & 1.0095 & 2.1082 & 2.3722 & 2.4307 & 3.3581 & 3.4861 & 3.7113 \\
\hline & 5 & 1.2390 & 0.5785 & 2.1733 & 1.4049 & 1.2301 & 2.1244 & 2.6004 & 2.6517 & 3.4307 & 3.7493 & 3.9922 \\
\hline \multirow{5}{*}{$\pi / 2$} & 1 & 0.2254 & 0.0567 & 0.4832 & 0.2681 & 0.3884 & 0.3526 & 0.6733 & 0.7128 & 1.3685 & 1.5437 & 1.6240 \\
\hline & 2 & 0.3474 & 0.0805 & 0.5981 & 0.5820 & 0.4298 & 0.6070 & 1.1785 & 1.2212 & 1.3716 & 1.5710 & 1.7063 \\
\hline & 3 & 0.5669 & 0.1808 & 0.6773 & 0.6731 & 0.7881 & 0.6711 & 1.1952 & 1.3154 & 1.5569 & 1.6229 & 1.9719 \\
\hline & 4 & 0.6454 & 0.2538 & 1.0277 & 0.9620 & 0.8104 & 0.7865 & 1.3272 & 1.4204 & 1.5885 & 1.7715 & 2.0406 \\
\hline & 5 & 0.9703 & 0.4872 & 1.1454 & 1.1509 & 1.0139 & 1.1836 & 1.5446 & 1.6729 & 1.6881 & 2.1317 & 2.3737 \\
\hline \multirow{5}{*}{$\pi / 3$} & 1 & 0.2126 & 0.0580 & 0.2379 & 0.2819 & 0.4292 & 0.2769 & 0.6692 & 0.6938 & 0.8346 & 0.9442 & 1.0676 \\
\hline & 2 & 0.2606 & 0.0803 & 0.2840 & 0.5475 & 0.4689 & 0.3002 & 0.8560 & 0.9925 & 0.8559 & 0.9845 & 1.2500 \\
\hline & 3 & 0.5323 & 0.1718 & 0.4670 & 0.7092 & 0.7338 & 0.3740 & 0.9431 & 1.0621 & 0.9811 & 1.0750 & 1.3691 \\
\hline & 4 & 0.5418 & 0.2511 & 0.5593 & 0.8415 & 0.8164 & 0.7040 & 0.9442 & 1.2147 & 1.0527 & 1.2809 & 1.5463 \\
\hline & 5 & 0.8533 & 0.4427 & 0.6721 & 1.0935 & 0.9915 & 0.9231 & 1.1936 & 1.3363 & 1.1993 & 1.3517 & 1.5777 \\
\hline \multirow{5}{*}{$\pi / 6$} & 1 & 0.2444 & 0.0815 & 0.1090 & 0.4494 & 0.5350 & 0.2052 & 0.5474 & 0.7132 & 0.4876 & 0.5474 & 0.8128 \\
\hline & 2 & 0.2548 & 0.1004 & 0.1109 & 0.5805 & 0.5673 & 0.2533 & 0.6518 & 0.7898 & 0.6067 & 0.7073 & 0.9307 \\
\hline & 3 & 0.5319 & 0.2163 & 0.2741 & 0.7805 & 0.8465 & 0.3545 & 0.6736 & 0.9967 & 0.7137 & 0.8791 & 1.0796 \\
\hline & 4 & 0.5928 & 0.2856 & 0.2829 & 0.9844 & 0.8830 & 0.6073 & 0.8265 & 1.2215 & 0.7504 & 0.9041 & 1.2947 \\
\hline & 5 & 0.6822 & 0.4981 & 0.4473 & 1.0485 & 1.0250 & 0.6499 & 1.0942 & 1.2959 & 0.7543 & 1.1170 & 1.3992 \\
\hline
\end{tabular}

$$
\begin{aligned}
& \gamma_{y z}=\frac{\partial w}{\partial y}+\frac{\partial v}{\partial z} \\
& \gamma_{x z}=\frac{\partial w}{\partial x}+\frac{\partial u}{\partial z}
\end{aligned}
$$

and, from Figure 1(c), the strain-displacement relations for open cylindrical shells are defined as

$$
\begin{aligned}
\varepsilon_{x} & =\frac{\partial u}{\partial x}, \\
\varepsilon_{\theta} & =\frac{w}{z}+\frac{\partial v}{z \partial \theta}, \\
\varepsilon_{z} & =\frac{\partial w}{\partial z}, \\
\gamma_{\theta z} & =\frac{\partial w}{z \partial \theta}+\frac{\partial v}{\partial z}-\frac{v}{z}, \\
\gamma_{x z} & =\frac{\partial w}{\partial x}+\frac{\partial u}{\partial z}, \\
\gamma_{x \theta} & =\frac{\partial u}{z \partial \theta}+\frac{\partial v}{\partial x} .
\end{aligned}
$$

Similarly, according to Figure 1(d), the strain-displacement relations for open spherical shells are given as

$$
\varepsilon_{\phi}=\frac{\partial u}{z \partial \phi}+\frac{w}{z}
$$

$$
\begin{aligned}
\varepsilon_{\theta} & =\frac{c_{\phi} u}{z}+\frac{1}{s_{\phi}} \frac{\partial v}{z \partial \theta}+\frac{w}{z}, \\
\varepsilon_{z} & =\frac{\partial w}{\partial z} \\
\gamma_{\theta z} & =\frac{\partial v}{\partial z}+\frac{1}{z}\left(\frac{1}{s_{\phi}} \frac{\partial w}{\partial \theta}-v\right), \\
\gamma_{\phi z} & =\frac{\partial u}{\partial z}+\frac{1}{z}\left(\frac{\partial w}{\partial \phi}-u\right), \\
\gamma_{\phi \theta} & =\frac{1}{s_{\phi}} \frac{\partial u}{z \partial \theta}+\frac{\partial v}{z \partial \phi}-\frac{c_{\phi} v}{z} .
\end{aligned}
$$

\section{B. Stiffness and Mass Matrices for Cylindrical and Spherical Shells}

The displacement components can be rewritten in the vector form as

$$
\begin{gathered}
u(\alpha, \beta, z, t)=U(\alpha, \beta, z) e^{j \omega t}=\mathbf{U G}^{u} e^{j \omega t}, \\
v(\alpha, \beta, z, t)=V(\alpha, \beta, z) e^{j \omega t}=\mathbf{V G}^{v} e^{j \omega t}, \\
w(\alpha, \beta, z, t)=W(\alpha, \beta, z) e^{j \omega t}=\mathbf{W G}^{w} e^{j \omega t},
\end{gathered}
$$



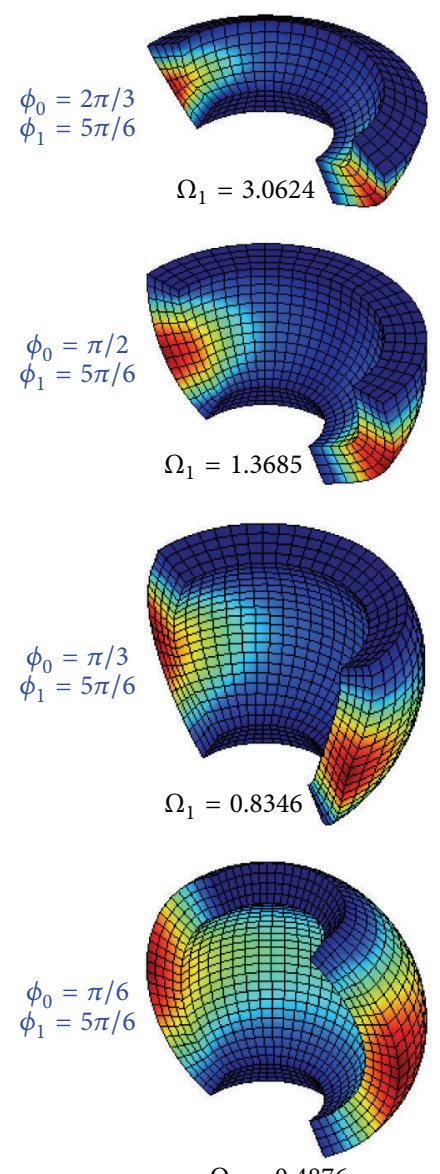

$\Omega_{1}=0.4876$
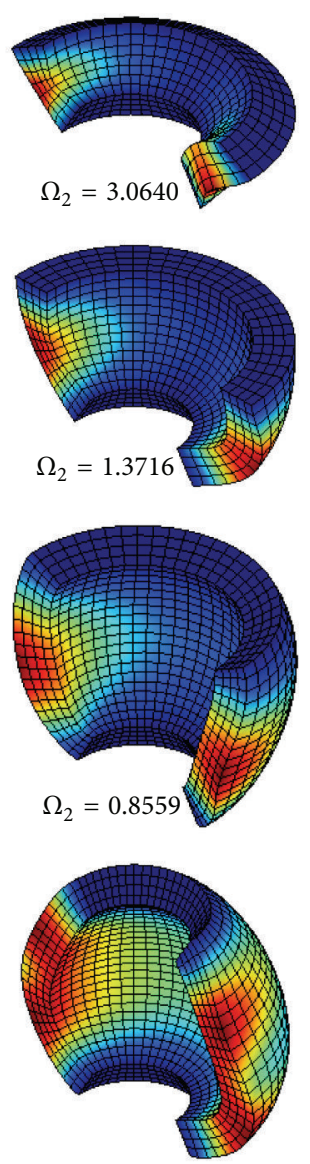

$\Omega_{2}=0.6067$
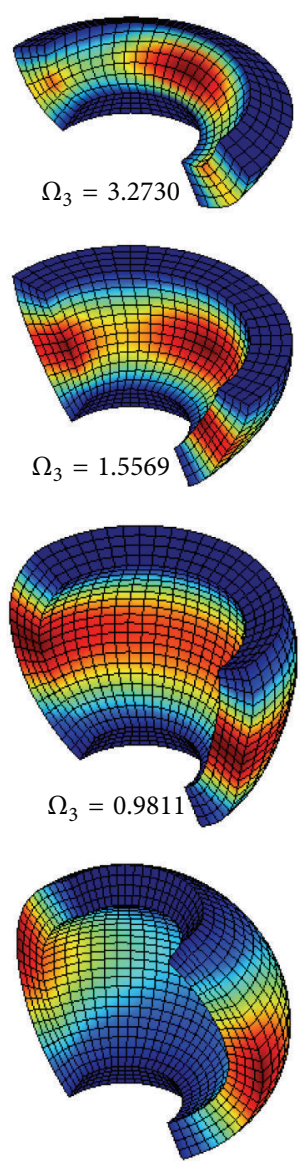

$\Omega_{3}=0.7137$
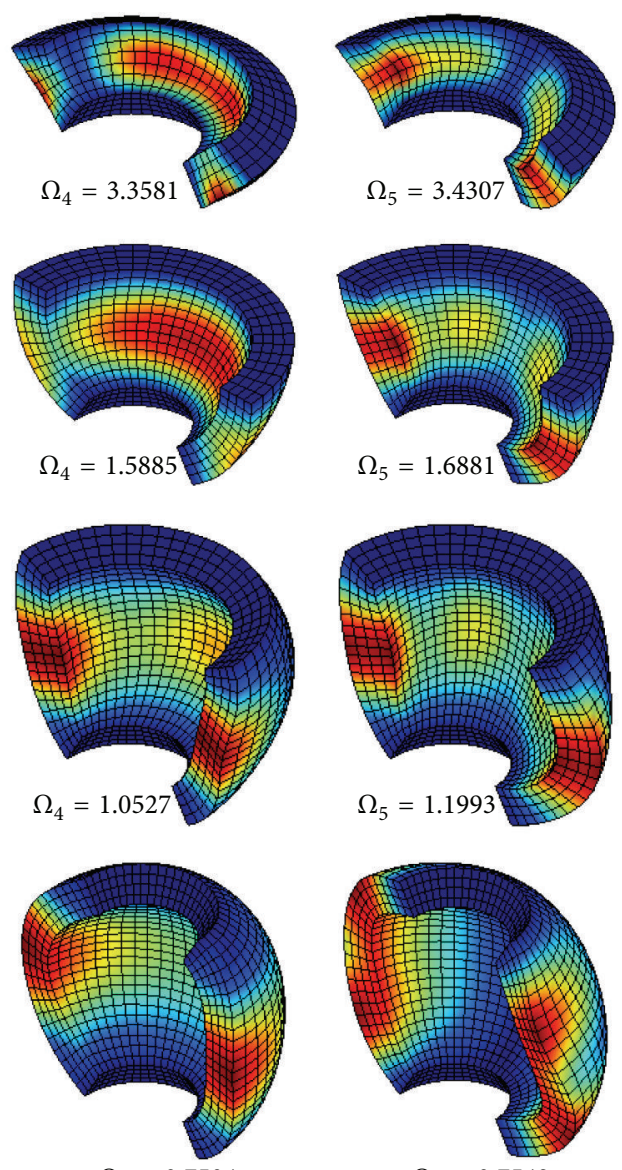

$\Omega_{4}=0.7504$

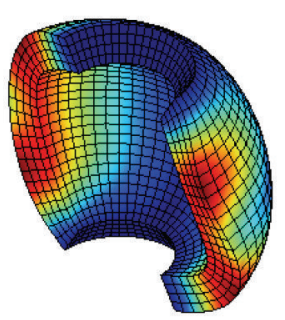

$\Omega_{5}=0.7543$

FIGURE 7: Mode shapes of an isotropic spherical shell with CFCF boundary conditions and various meridional dimensions.

where

$$
\begin{aligned}
\mathbf{U} & =\left[\cos \lambda_{0} \alpha \cos \lambda_{0} \beta \cos \lambda_{0} z, \ldots, \cos \lambda_{m} \alpha \cos \lambda_{n} \beta\right. \\
\cdot & \cos \lambda_{q} z, \ldots, \cos \lambda_{M} \alpha \cos \lambda_{N} \beta \cos \lambda_{\mathrm{Q}} z, \zeta_{1}(\alpha) \\
& \cdot \cos \lambda_{0} \beta \cos \lambda_{0} z, \ldots, \zeta_{k}(\alpha) \cos \lambda_{n} \beta \\
& \cdot \cos \lambda_{q} z, \ldots, \zeta_{2}(\alpha) \cos \lambda_{N} \beta \cos \lambda_{\mathrm{Q}} z, \zeta_{1}(\beta) \cos \lambda_{0} \alpha \\
& \cdot \cos \lambda_{0} z, \ldots, \zeta_{k}(\beta) \cos \lambda_{m} \alpha \cos \lambda_{q} z, \ldots, \zeta_{2}(\beta) \\
& \cdot \cos \lambda_{M} \alpha \cos \lambda_{\mathrm{Q}} z, \zeta_{1}(z) \cos \lambda_{0} \alpha \\
& \cdot \cos \lambda_{0} \beta, \ldots, \zeta_{k}(z) \cos \lambda_{m} \alpha \cos \lambda_{n} \beta, \ldots, \zeta_{2}(z) \\
& \left.\cdot \cos \lambda_{M} \alpha \cos \lambda_{N} \beta\right], \\
\mathbf{V} & =\mathbf{W}=\mathbf{U} .
\end{aligned}
$$

Thus, the stiffness and mass matrixes of an open cylindrical shell can be written as

$$
\begin{gathered}
\mathbf{K}_{u \boldsymbol{u}}=\iiint\left\{C_{11} \frac{\partial \mathbf{U}^{T}}{\partial x} \frac{\partial \mathbf{U}}{\partial x}+C_{55} \frac{\partial \mathbf{U}^{T}}{\partial z} \frac{\partial \mathbf{U}}{\partial z}\right. \\
\left.+C_{66} \frac{\partial \mathbf{U}^{T}}{z^{2} \partial \theta} \frac{\partial \mathbf{U}}{\partial \theta}\right\} d V+\iint\left\{\left.k_{x 0}^{u} \mathbf{U}^{T} \mathbf{U}\right|_{x=0}\right.
\end{gathered}
$$

$$
\begin{gathered}
\left.+\left.k_{x 1}^{u} \mathbf{U}^{T} \mathbf{U}\right|_{x=L}\right\} d S_{1}+\iint\left\{\left.k_{\theta 0}^{u} \mathbf{U}^{T} \mathbf{U}\right|_{\theta=0}\right. \\
\left.+\left.k_{\theta 1}^{u} \mathbf{U}^{T} \mathbf{U}\right|_{\theta=\theta_{0}}\right\} d S_{2}, \\
\mathbf{K}_{u v}=\iiint\left\{C_{12} \frac{\partial \mathbf{V}^{T}}{z \partial \theta} \frac{\partial \mathbf{U}}{\partial x}+C_{55} \frac{\partial \mathbf{V}^{T}}{\partial x} \frac{\partial \mathbf{U}}{z \partial \theta}\right\} d V, \\
\mathbf{K}_{u w}=\iiint\left\{C_{12} \mathbf{W}^{T} \frac{\partial \mathbf{U}}{z \partial x}+C_{13} \frac{\partial \mathbf{W}^{T}}{\partial z} \frac{\partial \mathbf{U}}{\partial x}\right. \\
\left.+C_{55} \frac{\partial \mathbf{W}^{T}}{\partial x} \frac{\partial \mathbf{U}}{\partial z}\right\} d V, \\
\quad=\iiint\left\{C_{22} \frac{\partial \mathbf{V}^{T}}{z^{2} \partial \theta} \frac{\partial \mathbf{V}}{\partial \theta}\right. \\
+C_{44}\left(\frac{\partial \mathbf{V}^{T}}{\partial z} \frac{\partial \mathbf{V}}{\partial z}-\frac{\partial \mathbf{V}^{T}}{z \partial z} \mathbf{V}-\mathbf{V}^{T} \frac{\partial \mathbf{V}}{z \partial z}+\frac{\mathbf{V}^{T} \mathbf{V}}{z^{2}}\right) \\
\left.+\left.k_{x 1}^{v} \mathbf{V}^{T} \mathbf{V}\right|_{x=L} \frac{\partial \mathbf{V}^{T}}{\partial x} \frac{\partial \mathbf{V}}{\partial x}\right\} d V+\iint\left\{s_{1}^{v}+\left.\int \mathbf{V}^{T} \mathbf{V}\right|_{x=0}\left\{\left.k_{\theta 0}^{v} \mathbf{V}^{T} \mathbf{V}\right|_{\theta=0}\right.\right.
\end{gathered}
$$




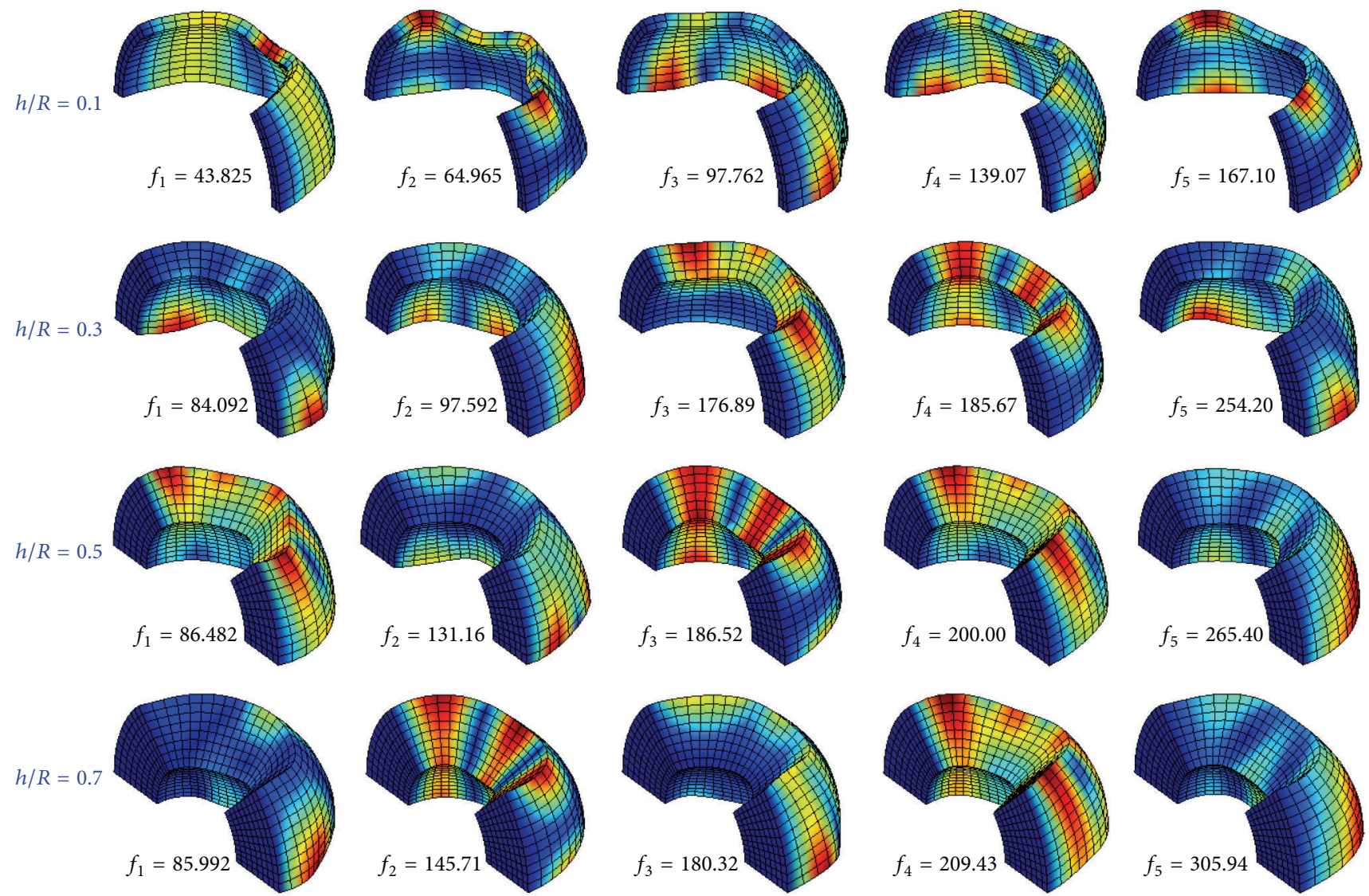

FIGURE 8: Mode shapes of an orthotropic spherical shell with FCFC boundary conditions and various thickness-radius ratios.

$$
\begin{aligned}
& \left.+\left.k_{\theta 1}^{v} \mathbf{V}^{T} \mathbf{V}\right|_{\theta=\theta_{0}}\right\} d S_{2}, \\
& \mathbf{K}_{v w}=\iiint\left\{\frac{C_{22}}{z^{2}} \mathbf{W}^{T} \frac{\partial \mathbf{V}}{\partial \theta}+\frac{C_{23}}{z} \frac{\partial \mathbf{W}^{T}}{\partial z} \frac{\partial \mathbf{V}}{\partial \theta}\right. \\
& \left.+\frac{C_{44}}{z}\left(\frac{\partial \mathbf{W}^{T}}{\partial \theta} \frac{\partial \mathbf{V}}{\partial z}-\frac{\partial \mathbf{W}^{T}}{z \partial \theta} \mathbf{V}\right)\right\} d V \\
& \mathbf{K}_{w w}=\iiint\left\{C_{22} \frac{\mathbf{W}^{T} \mathbf{W}}{z^{2}}+C_{23}\left(\mathbf{W}^{T} \frac{\partial \mathbf{W}}{z \partial z}+\frac{\partial \mathbf{W}^{T}}{z \partial z} \mathbf{W}\right)\right. \\
& +C_{33} \frac{\partial \mathbf{W}^{T}}{\partial z} \frac{\partial \mathbf{W}}{\partial z}+C_{44} \frac{\partial \mathbf{W}^{T}}{z^{2} \partial \theta} \frac{\partial \mathbf{W}}{\partial \theta} \\
& \left.+C_{55} \frac{\partial \mathbf{W}^{T}}{\partial x} \frac{\partial \mathbf{W}}{\partial x}\right\} d V+\iint\left\{\left.k_{x 0}^{w} \mathbf{W}^{T} \mathbf{W}\right|_{x=0}\right. \\
& \left.+\left.k_{x 1}^{w} \mathbf{W}^{T} \mathbf{W}\right|_{x=L}\right\} d S_{1}+\iint\left\{\left.k_{\theta 0}^{w} \mathbf{W}^{T} \mathbf{W}\right|_{\theta=0}\right. \\
& \left.+\left.k_{\theta 1}^{w} \mathbf{W}^{T} \mathbf{W}\right|_{\theta=\theta_{0}}\right\} d S_{2}, \\
& \mathbf{M}_{u u}=\iiint\left\{\rho \mathbf{U}^{T} \mathbf{U}\right\} d V,
\end{aligned}
$$

$$
\begin{aligned}
& \mathbf{M}_{v v}=\iiint\left\{\rho \mathbf{V}^{T} \mathbf{V}\right\} d V, \\
& \mathbf{M}_{w w}=\iiint\left\{\rho \mathbf{W}^{T} \mathbf{W}\right\} d V,
\end{aligned}
$$

where $d V=z d z d \theta d x, d S_{1}=z d z d \theta$, and $d S_{2}=d z d x$. Similarly, the stiffness and mass matrices of an open spherical shell are

$$
\begin{aligned}
& \mathbf{K}_{u u}=\iiint\left\{\frac{C_{11}}{z^{2}} \frac{\partial \mathbf{U}^{T}}{\partial \phi} \frac{\partial \mathbf{U}}{\partial \phi}+\frac{C_{13} c_{\phi}}{z^{2}}\left(\frac{\partial \mathbf{U}^{T}}{\partial \phi} \mathbf{U}\right.\right. \\
& \left.+\mathbf{U}^{T} \frac{\partial \mathbf{U}}{\partial \phi}\right)+\frac{C_{22} c_{\phi}^{2}}{z^{2}} \mathbf{U}^{T} \mathbf{U}+C_{55}\left(\frac{\mathbf{U}^{T} \mathbf{U}}{z^{2}}\right. \\
& \left.-\frac{\mathbf{U}^{T} \partial \mathbf{U}}{z \partial z}-\frac{\partial \mathbf{U}^{T}}{z \partial z} \mathbf{U}+\frac{\partial \mathbf{U}^{T}}{\partial z} \frac{\partial \mathbf{U}}{\partial z}\right)+\frac{C_{66}}{s_{\phi}^{2} z^{2}} \frac{\partial \mathbf{U}^{T}}{\partial \theta} \\
& \left.+\frac{\partial \mathbf{U}}{\partial \theta}\right\} d V+\iint\left\{\left.k_{\phi 0}^{u} \mathbf{U}^{T} \mathbf{U}\right|_{\phi=\phi_{0}}+\left.k_{\phi 1}^{u} \mathbf{U}^{T} \mathbf{U}\right|_{\phi=\phi_{1}}\right\} d S_{1} \\
& +\iint\left\{\left.k_{\theta 0}^{u} \mathbf{U}^{T} \mathbf{U}\right|_{\theta=0}+\left.k_{\theta 1}^{u} \mathbf{U}^{T} \mathbf{U}\right|_{\theta=\theta_{0}}\right\} d S_{2},
\end{aligned}
$$




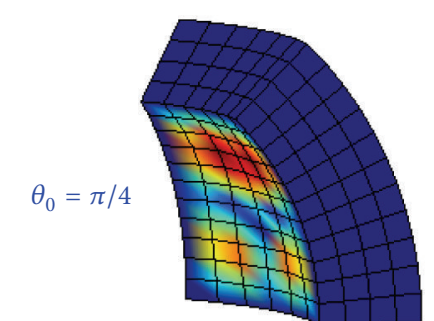

$f_{1}=1186.0$
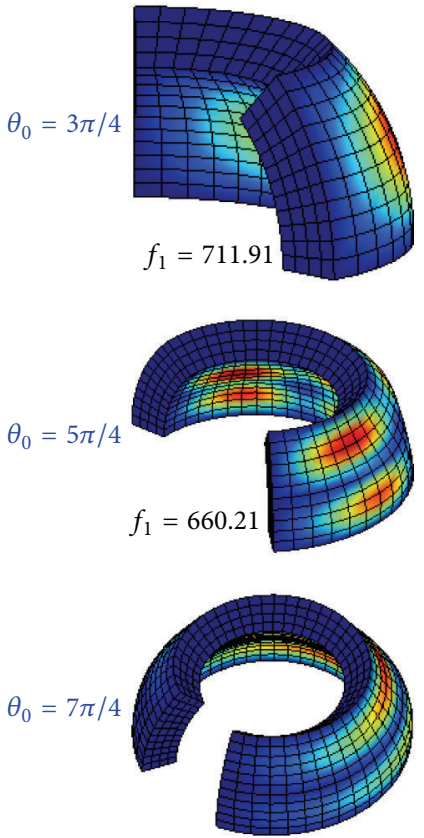

$f_{1}=645.28$

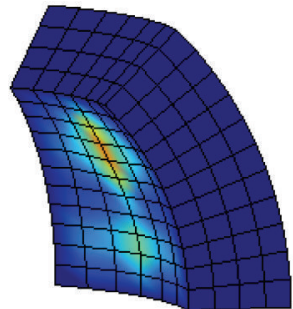

$f_{2}=1235.9$
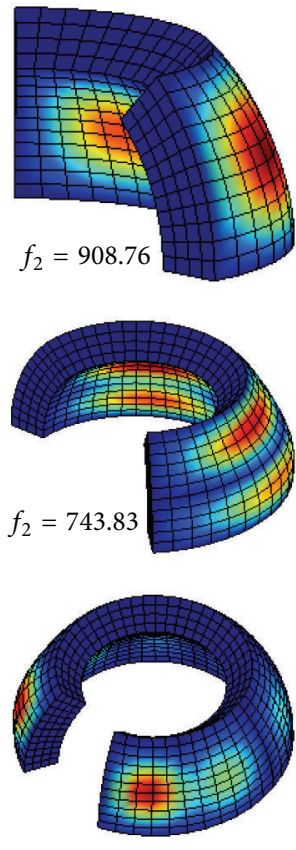

$f_{2}=690.18$

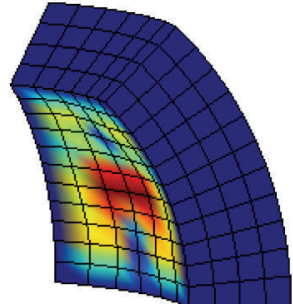

$f_{3}=1367.2$
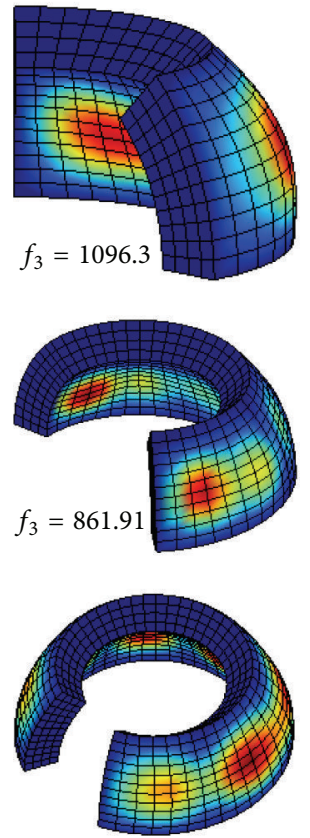

$f_{3}=758.25$

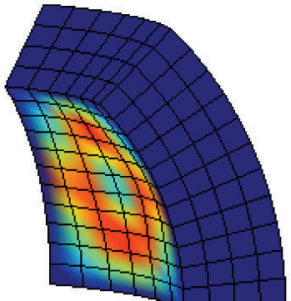

$f_{4}=1519.4$
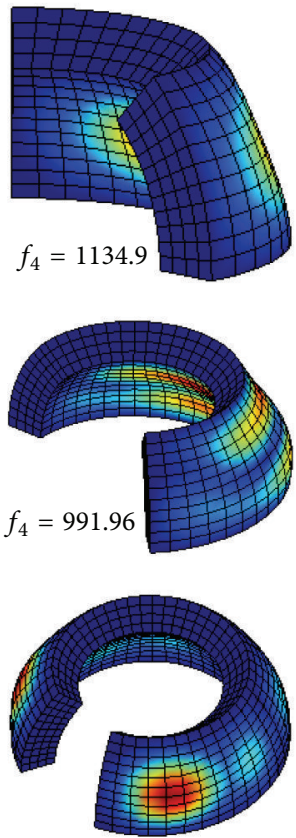

$f_{4}=842.27$

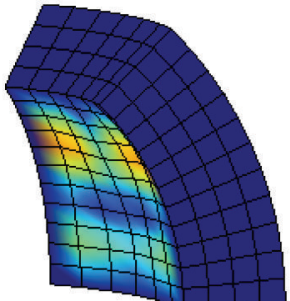

$f_{5}=1647.8$
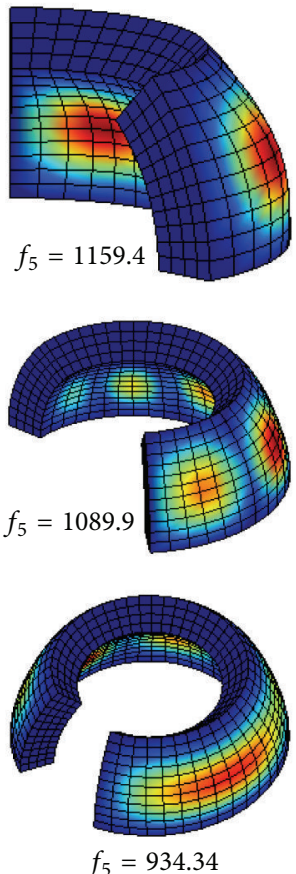

FIGURE 9: Mode shapes of an orthotropic spherical shell with CCCC boundary conditions and various circumferential dimensions.

$$
\begin{aligned}
& \mathbf{K}_{u v}=\iiint\left\{\frac{C_{12}}{z^{2} s_{\phi}} \frac{\partial \mathbf{V}^{T}}{\partial \theta} \frac{\partial \mathbf{U}}{\partial \phi}+\frac{C_{22} c_{\phi}}{z^{2} s_{\phi}} \frac{\partial \mathbf{V}^{T}}{\partial \theta} \mathbf{U}\right. \\
& \left.-\frac{\partial \mathbf{V}^{T}}{\partial z} \frac{\mathbf{V}}{z}-\frac{\mathbf{V}^{T}}{z} \frac{\partial \mathbf{V}}{\partial z}+\frac{\mathbf{V}^{T} \mathbf{V}}{z^{2}}\right)+\frac{C_{66}}{z^{2}}\left(\frac{\partial \mathbf{V}^{T}}{\partial \phi} \frac{\partial \mathbf{V}}{\partial \phi}\right. \\
& \left.+\frac{C_{66}}{z^{2} s_{\phi}}\left(\frac{\partial \mathbf{V}^{T}}{\partial \phi} \frac{\partial \mathbf{U}}{\partial \theta}-c_{\phi} \mathbf{V}^{T} \frac{\partial \mathbf{U}}{\partial \theta}\right)\right\} d V, \\
& \left.\left.-c_{\phi} \frac{\partial \mathbf{V}^{T}}{\partial \phi} \mathbf{V}-c_{\phi} \mathbf{V}^{T} \frac{\partial \mathbf{V}}{\partial \phi}+c_{\phi}^{2} \mathbf{V}^{T} \mathbf{V}\right)\right\} d V \\
& \mathbf{K}_{u w}=\iiint\left\{\frac{C_{11}}{z^{2}} \mathbf{W}^{T} \frac{\partial \mathbf{U}}{\partial \phi}+C_{12}\left(\frac{c_{\phi}}{z^{2}} \mathbf{W}^{T} \mathbf{U}\right.\right. \\
& +\iint\left\{\left.k_{\phi 0}^{v} \mathbf{V}^{T} \mathbf{V}\right|_{\phi=\phi_{0}}+\left.k_{\phi 1}^{v} \mathbf{V}^{T} \mathbf{V}\right|_{\phi=\phi_{1}}\right\} d S_{1} \\
& \left.+\frac{c_{\phi}}{z^{2} s_{\phi}} \mathbf{W}^{T} \frac{\partial \mathbf{U}}{\partial \phi}\right)+C_{13} \frac{\partial \mathbf{W}^{T}}{z \partial z} \frac{\partial \mathbf{U}}{\partial \phi}+\frac{C_{22} c_{\phi}}{z^{2}} \mathbf{W}^{T} \mathbf{U} \\
& +\iint\left\{\left.k_{\theta 0}^{v} \mathbf{V}^{T} \mathbf{V}\right|_{\theta=0}+\left.k_{\theta 1}^{v} \mathbf{V}^{T} \mathbf{V}\right|_{\theta=\theta_{0}}\right\} d S_{2}, \\
& +\frac{C_{23} c_{\phi}}{z} \frac{\partial \mathbf{W}^{T}}{\partial z} \mathbf{U}+C_{55}\left(\frac{\partial \mathbf{W}^{T}}{z \partial \phi} \frac{\partial \mathbf{U}}{\partial z}\right. \\
& \left.\left.-\frac{\partial \mathbf{W}^{T}}{z^{2} s_{\phi} \partial \theta} \frac{\partial \mathbf{U}}{\partial \phi}\right)\right\} d V \\
& \mathbf{K}_{v w}=\iiint\left\{\frac{C_{12}}{s_{\phi} z^{2}} \mathbf{W}^{T} \frac{\partial \mathbf{V}}{\partial \theta}+\frac{C_{22}}{s_{\phi} z^{2}} \mathbf{W}^{T} \frac{\partial \mathbf{V}}{\partial \theta}+\frac{C_{23}}{s_{\phi} z} \frac{\partial \mathbf{W}^{T}}{\partial z}\right. \\
& \left.\frac{\partial \mathbf{V}}{\partial \theta}+\frac{C_{44}}{s_{\phi} z}\left(\frac{\partial \mathbf{W}^{T}}{\partial \theta} \frac{\partial \mathbf{V}}{\partial z}-\frac{\partial \mathbf{W}^{T}}{z \partial \theta} \mathbf{V}\right)\right\} d V, \\
& \mathbf{K}_{v v}=\iiint\left\{\frac{C_{22}}{z^{2} s_{\phi}^{2}} \frac{\partial \mathbf{V}^{T}}{\partial \theta} \frac{\partial \mathbf{V}}{\partial \theta}+C_{44}\left(\frac{\partial \mathbf{V}^{T}}{\partial z} \frac{\partial \mathbf{V}}{\partial z}\right.\right. \\
& \mathbf{K}_{w w}=\iiint\left\{\left(\frac{C_{11}}{z^{2}}+\frac{2 C_{12}}{z^{2}}+\frac{C_{22}}{z^{2}}\right) \mathbf{W}^{T} \mathbf{W}+\left(\frac{C_{13}}{z}\right.\right.
\end{aligned}
$$


TABLE 15: The first five frequencies $(\mathrm{Hz})$ of an open orthotropic spherical shell with various boundary conditions and thickness-radius ratios $\left(\phi_{0}=\pi / 4, \phi_{1}=\pi / 2, R=1 \mathrm{~m}\right.$, and $\left.\theta_{0}=\pi\right)$.

\begin{tabular}{|c|c|c|c|c|c|c|c|c|c|c|c|c|}
\hline \multirow{2}{*}{$h / R$} & \multirow{2}{*}{ Mode } & \multicolumn{11}{|c|}{ Boundary conditions } \\
\hline & & FFFF & FFFC & CFFFF & FSFS & FCFC & SFSF & SSSS & SCSC & CFCF & CSCS & CCCC \\
\hline \multirow{5}{*}{0.1} & 1 & 19.896 & 4.7150 & 145.92 & 26.597 & 43.825 & 92.211 & 204.15 & 206.87 & 637.75 & 640.79 & 687.84 \\
\hline & 2 & 38.600 & 7.1240 & 151.20 & 59.003 & 64.965 & 199.05 & 364.74 & 367.14 & 681.48 & 686.93 & 808.17 \\
\hline & 3 & 55.399 & 15.160 & 153.30 & 71.455 & 97.762 & 340.84 & 417.88 & 420.49 & 799.24 & 804.31 & 947.86 \\
\hline & 4 & 79.201 & 30.990 & 171.24 & 130.15 & 139.07 & 344.15 & 430.75 & 437.31 & 932.69 & 937.60 & 1020.7 \\
\hline & 5 & 106.85 & 49.233 & 187.33 & 145.77 & 167.10 & 371.91 & 434.46 & 442.39 & 1001.0 & 1008.7 & 1047.7 \\
\hline \multirow{5}{*}{0.2} & 1 & 33.414 & 7.9209 & 214.98 & 45.180 & 72.592 & 96.974 & 207.03 & 210.15 & 633.78 & 636.74 & 684.33 \\
\hline & 2 & 69.721 & 12.651 & 216.11 & 93.424 & 80.087 & 200.08 & 371.20 & 375.03 & 677.97 & 683.38 & 807.60 \\
\hline & 3 & 93.999 & 25.374 & 226.26 & 121.23 & 146.55 & 370.53 & 517.60 & 521.02 & 798.38 & 803.76 & 963.56 \\
\hline & 4 & 132.28 & 47.581 & 230.62 & 219.47 & 176.25 & 457.82 & 528.68 & 536.52 & 946.63 & 953.19 & 1072.6 \\
\hline & 5 & 177.78 & 80.265 & 263.47 & 233.78 & 233.90 & 460.49 & 533.26 & 539.54 & 1041.4 & 1057.8 & 1113.8 \\
\hline \multirow{10}{*}{0.3} & 1 & 47.187 & 11.234 & 241.04 & 63.466 & 84.092 & 97.431 & 205.91 & 209.10 & 626.26 & 629.50 & 676.95 \\
\hline & 2 & 94.608 & 16.780 & 244.82 & 109.78 & 97.592 & 198.64 & 368.42 & 372.44 & 669.88 & 676.00 & 800.45 \\
\hline & 3 & 129.46 & 35.164 & 254.48 & 166.60 & 176.89 & 367.72 & 540.50 & 543.66 & 789.91 & 796.76 & 961.81 \\
\hline & 4 & 157.36 & 55.245 & 254.74 & 250.56 & 185.67 & 495.01 & 557.49 & 561.94 & 941.27 & 952.43 & 1092.2 \\
\hline & 5 & 237.51 & 106.95 & 277.95 & 270.09 & 254.20 & 499.55 & 572.32 & 582.43 & 1040.1 & 1079.2 & 1132.3 \\
\hline & $1^{\mathrm{A}}$ & 47.232 & 11.217 & 242.75 & 63.374 & 84.068 & 97.543 & 206.35 & 209.48 & 627.38 & 631.29 & 678.57 \\
\hline & $2^{\mathrm{A}}$ & 95.033 & 16.795 & 246.89 & 110.14 & 97.313 & 198.87 & 369.20 & 373.16 & 670.22 & 677.73 & 801.91 \\
\hline & $3^{\mathrm{A}}$ & 129.90 & 35.127 & 255.38 & 167.11 & 176.99 & 368.44 & 542.07 & 545.12 & 790.89 & 798.61 & 963.97 \\
\hline & $4^{\mathrm{A}}$ & 157.94 & 55.319 & 256.78 & 251.11 & 185.96 & 499.94 & 563.57 & 567.98 & 943.59 & 955.28 & 1097.9 \\
\hline & $5^{\mathrm{A}}$ & 238.72 & 106.97 & 279.74 & 271.36 & 254.70 & 504.74 & 578.49 & 588.35 & 1046.1 & 1084.8 & 1140.1 \\
\hline \multirow{5}{*}{0.4} & 1 & 60.205 & 14.431 & 251.07 & 80.237 & 85.760 & 96.770 & 203.49 & 206.68 & 615.23 & 618.93 & 665.74 \\
\hline & 2 & 113.00 & 19.698 & 253.75 & 116.83 & 117.06 & 196.17 & 363.47 & 367.52 & 657.54 & 664.82 & 787.70 \\
\hline & 3 & 160.03 & 43.922 & 259.74 & 204.51 & 187.57 & 362.74 & 533.94 & 536.72 & 775.23 & 784.31 & 949.69 \\
\hline & 4 & 164.11 & 58.904 & 270.40 & 257.72 & 191.75 & 507.17 & 578.32 & 583.76 & 924.19 & 941.97 & 1098.0 \\
\hline & 5 & 264.31 & 126.28 & 299.87 & 281.51 & 261.86 & 515.22 & 597.45 & 609.14 & 1021.8 & 1088.6 & 1129.6 \\
\hline \multirow{5}{*}{0.5} & 1 & 72.074 & 17.420 & 250.27 & 94.752 & 86.482 & 95.525 & 200.21 & 203.37 & 600.93 & 605.18 & 650.82 \\
\hline & 2 & 125.55 & 21.692 & 255.44 & 119.83 & 131.16 & 192.94 & 356.93 & 360.93 & 641.35 & 649.95 & 769.50 \\
\hline & 3 & 162.84 & 51.465 & 266.92 & 233.65 & 186.52 & 356.18 & 523.57 & 526.18 & 755.11 & 766.45 & 927.68 \\
\hline & 4 & 185.83 & 60.700 & 279.17 & 262.21 & 200.00 & 506.90 & 591.05 & 597.40 & 898.66 & 921.41 & 1086.3 \\
\hline & 5 & 265.90 & 132.30 & 316.99 & 285.08 & 265.40 & 519.90 & 605.18 & 625.92 & 997.61 & 1079.3 & 1112.8 \\
\hline
\end{tabular}

$1^{\mathrm{A}}$ to $5^{\mathrm{A}}$ : results obtained by ANSYS.

$$
\begin{aligned}
& \left.+\frac{C_{23}}{z}\right)\left(\mathbf{W}^{T} \frac{\partial \mathbf{W}}{\partial z}+\frac{\partial \mathbf{W}^{T}}{\partial z} \mathbf{W}\right)+C_{33} \frac{\partial \mathbf{W}^{T}}{\partial z} \frac{\partial \mathbf{W}}{\partial z} \\
& \left.+C_{44} \frac{\partial \mathbf{W}^{T}}{s_{\phi}^{2} z^{2} \partial \theta} \frac{\partial \mathbf{W}}{\partial \theta}+\frac{C_{55}}{z^{2}} \frac{\partial \mathbf{W}^{T}}{\partial \phi} \frac{\partial \mathbf{W}}{\partial \phi}\right\} d V \\
& +\iint\left\{\left.k_{\phi 0}^{w} \mathbf{W}^{T} \mathbf{W}\right|_{\phi=\phi_{0}}+\left.k_{\phi 1}^{w} \mathbf{W}^{T} \mathbf{W}\right|_{\phi=\phi_{1}}\right\} d S_{1} \\
& +\iint\left\{\left.k_{\theta 0}^{w} \mathbf{W}^{T} \mathbf{W}\right|_{\theta=0}+\left.k_{\theta 1}^{w} \mathbf{W}^{T} \mathbf{W}\right|_{\theta=\theta_{0}}\right\} d S_{2}, \\
& \mathbf{M}_{u u}=\iiint\left\{\rho \mathbf{U}^{T} \mathbf{U}\right\} d V,
\end{aligned}
$$$$
\begin{aligned}
& \mathbf{M}_{v v}=\iiint\left\{\rho \mathbf{V}^{T} \mathbf{V}\right\} d V, \\
& \mathbf{M}_{w w}=\iiint\left\{\rho \mathbf{W}^{T} \mathbf{W}\right\} d V,
\end{aligned}
$$

where $d V=z^{2} s_{\phi} d z d \theta d \phi, d S_{1}=z s_{\phi} d z d \theta$, and $d S_{2}=$ $z d z d \phi$.

\section{Conflict of Interests}

The authors declare that there is no conflict of interests regarding the publication of this paper. 
TABLE 16: The first five frequencies $(\mathrm{Hz})$ of an open orthotropic spherical shell with various boundary conditions and included angles $\left(\phi_{0}=\right.$ $\pi / 4, \phi_{1}=\pi / 2, R=1 \mathrm{~m}$, and $\left.h / R=0.3\right)$.

\begin{tabular}{|c|c|c|c|c|c|c|c|c|c|c|c|c|}
\hline \multirow{2}{*}{$\theta_{0}$} & \multirow{2}{*}{ Mode } & \multicolumn{11}{|c|}{ Boundary conditions } \\
\hline & & FFFF & FFFC & CFFFF & FSFS & FCFC & SFSF & SSSS & SCSC & CFCF & CSCS & CCCC \\
\hline \multirow{5}{*}{$\pi / 4$} & 1 & 443.51 & 130.71 & 222.38 & 293.54 & 536.71 & 66.396 & 629.50 & 728.26 & 611.21 & 629.50 & 1186.0 \\
\hline & 2 & 611.86 & 232.99 & 246.28 & 444.69 & 675.25 & 464.99 & 668.60 & 805.69 & 1020.2 & 1079.1 & 1235.9 \\
\hline & 3 & 882.78 & 286.55 & 279.19 & 617.48 & 714.50 & 507.72 & 731.49 & 1177.8 & 1036.7 & 1232.8 & 1367.2 \\
\hline & 4 & 902.67 & 477.56 & 637.73 & 702.69 & 937.59 & 689.20 & 1160.7 & 1259.3 & 1155.6 & 1294.8 & 1519.4 \\
\hline & 5 & 936.58 & 590.89 & 739.43 & 900.69 & 1079.2 & 738.47 & 1182.4 & 1362.2 & 1167.9 & 1302.6 & 1647.8 \\
\hline \multirow{5}{*}{$\pi / 2$} & 1 & 189.08 & 37.048 & 239.97 & 63.267 & 232.07 & 84.838 & 368.41 & 377.11 & 621.23 & 629.50 & 803.86 \\
\hline & 2 & 234.56 & 70.009 & 243.00 & 109.72 & 319.08 & 366.89 & 572.28 & 598.55 & 780.31 & 796.75 & 1106.6 \\
\hline & 3 & 362.22 & 140.87 & 263.46 & 293.61 & 367.59 & 492.89 & 629.50 & 706.99 & 1028.0 & 1079.1 & 1176.8 \\
\hline & 4 & 447.89 & 155.81 & 309.02 & 338.61 & 367.94 & 502.40 & 668.64 & 735.01 & 1056.8 & 1128.4 & 1199.3 \\
\hline & 5 & 512.70 & 280.64 & 493.30 & 444.73 & 495.53 & 606.52 & 731.50 & 831.07 & 1120.0 & 1225.0 & 1254.0 \\
\hline \multirow{5}{*}{$3 \pi / 4$} & 1 & 85.708 & 17.984 & 240.41 & 15.545 & 135.78 & 93.192 & 257.64 & 262.86 & 624.62 & 629.50 & 711.91 \\
\hline & 2 & 146.72 & 30.483 & 244.79 & 26.247 & 179.62 & 252.51 & 483.28 & 487.43 & 700.48 & 709.75 & 908.76 \\
\hline & 3 & 216.38 & 66.516 & 257.67 & 128.73 & 229.69 & 476.19 & 560.16 & 569.44 & 887.13 & 899.62 & 1096.3 \\
\hline & 4 & 222.58 & 88.782 & 264.21 & 214.11 & 284.71 & 496.05 & 595.97 & 616.53 & 1035.1 & 1079.1 & 1134.9 \\
\hline & 5 & 361.62 & 173.33 & 325.13 & 272.42 & 288.33 & 507.53 & 629.50 & 697.78 & 1062.2 & 1129.6 & 1159.4 \\
\hline \multirow{5}{*}{$\pi$} & 1 & 47.187 & 11.234 & 241.04 & 63.466 & 84.092 & 97.431 & 205.91 & 209.10 & 626.26 & 629.50 & 676.95 \\
\hline & 2 & 94.608 & 16.780 & 244.82 & 109.78 & 97.592 & 198.64 & 368.42 & 372.44 & 669.88 & 676.00 & 800.45 \\
\hline & 3 & 129.46 & 35.164 & 254.48 & 166.60 & 176.89 & 367.72 & 540.50 & 543.66 & 789.91 & 796.76 & 961.81 \\
\hline & 4 & 157.36 & 55.245 & 254.74 & 250.56 & 185.67 & 495.01 & 557.49 & 561.94 & 941.27 & 952.43 & 1092.2 \\
\hline & 5 & 237.51 & 106.95 & 277.95 & 270.09 & 254.20 & 499.55 & 572.32 & 582.43 & 1040.1 & 1079.2 & 1132.3 \\
\hline \multirow{5}{*}{$5 \pi / 4$} & 1 & 29.373 & 8.1735 & 242.94 & 6.1341 & 54.327 & 99.901 & 177.12 & 179.16 & 627.17 & 629.50 & 660.21 \\
\hline & 2 & 60.370 & 11.013 & 243.11 & 8.7143 & 56.663 & 169.06 & 301.12 & 304.42 & 655.30 & 659.69 & 743.83 \\
\hline & 3 & 82.483 & 21.409 & 250.47 & 32.422 & 122.29 & 299.61 & 437.16 & 440.06 & 737.18 & 741.85 & 861.91 \\
\hline & 4 & 119.80 & 35.084 & 252.42 & 55.852 & 125.78 & 436.10 & 556.76 & 559.23 & 851.69 & 857.42 & 991.96 \\
\hline & 5 & 157.24 & 64.758 & 265.43 & 101.21 & 206.84 & 498.30 & 563.86 & 569.32 & 972.10 & 982.99 & 1089.9 \\
\hline
\end{tabular}

TABLE 17: The first five frequencies $(\mathrm{Hz})$ of an open orthotropic spherical shell with various boundary conditions and meridian dimensions $\left(\phi_{1}=5 \pi / 6, \theta_{0}=\pi, R=1 \mathrm{~m}\right.$, and $\left.h / R=0.3\right)$.

\begin{tabular}{|c|c|c|c|c|c|c|c|c|c|c|c|c|}
\hline \multirow{2}{*}{$\phi_{0}$} & \multirow{2}{*}{ Mode } & \multicolumn{11}{|c|}{ Boundary conditions } \\
\hline & & FFFF & FFFC & CFFFF & FSFS & FCFC & SFSF & SSSS & SCSC & CFCF & CSCS & CCCC \\
\hline \multirow{5}{*}{$2 \pi / 3$} & 1 & 84.679 & 18.926 & 510.60 & 98.028 & 89.074 & 212.64 & 317.72 & 325.28 & 969.68 & 974.67 & 1027.7 \\
\hline & 2 & 146.31 & 25.038 & 521.72 & 152.51 & 154.01 & 291.59 & 490.39 & 502.82 & 1016.3 & 1026.4 & 1168.0 \\
\hline & 3 & 195.29 & 57.031 & 538.96 & 248.49 & 219.71 & 486.17 & 697.62 & 707.91 & 1147.7 & 1162.2 & 1326.9 \\
\hline & 4 & 222.27 & 72.206 & 597.15 & 319.22 & 255.89 & 696.35 & 887.99 & 892.37 & 1271.8 & 1312.8 & 1363.8 \\
\hline & 5 & 327.75 & 154.47 & 654.93 & 368.73 & 323.39 & 838.08 & 912.15 & 920.46 & 1303.4 & 1360.3 & 1382.2 \\
\hline \multirow{5}{*}{$\pi / 2$} & 1 & 57.626 & 14.203 & 228.35 & 71.121 & 102.60 & 117.91 & 214.91 & 228.01 & 456.22 & 460.47 & 533.24 \\
\hline & 2 & 99.202 & 22.128 & 243.09 & 154.35 & 110.66 & 209.78 & 368.11 & 376.89 & 523.97 & 531.36 & 700.27 \\
\hline & 3 & 148.74 & 45.715 & 258.66 & 179.30 & 205.12 & 309.18 & 404.08 & 422.12 & 685.82 & 693.36 & 872.42 \\
\hline & 4 & 178.28 & 68.778 & 316.42 & 253.59 & 220.52 & 317.77 & 442.10 & 454.90 & 830.34 & 859.53 & 884.69 \\
\hline & 5 & 256.56 & 126.55 & 349.70 & 307.45 & 263.17 & 380.38 & 460.47 & 502.97 & 846.00 & 882.39 & 923.62 \\
\hline \multirow{5}{*}{$\pi / 3$} & 1 & 59.987 & 15.391 & 120.01 & 76.167 & 111.87 & 98.56 & 197.31 & 203.81 & 263.67 & 267.89 & 362.54 \\
\hline & 2 & 76.001 & 22.205 & 123.49 & 145.39 & 125.12 & 135.77 & 266.90 & 310.24 & 353.31 & 359.87 & 530.43 \\
\hline & 3 & 148.26 & 45.380 & 169.24 & 188.24 & 192.03 & 147.10 & 267.89 & 311.52 & 491.00 & 529.88 & 555.30 \\
\hline & 4 & 152.12 & 68.610 & 187.82 & 224.49 & 224.19 & 208.90 & 302.45 & 349.51 & 495.19 & 535.24 & 572.28 \\
\hline & 5 & 264.29 & 120.70 & 261.95 & 301.40 & 255.38 & 280.38 & 374.50 & 413.92 & 535.13 & 577.62 & 638.34 \\
\hline \multirow{5}{*}{$\pi / 6$} & 1 & 71.868 & 23.031 & 57.238 & 119.02 & 145.90 & 69.88 & 155.36 & 228.09 & 151.76 & 155.36 & 288.40 \\
\hline & 2 & 86.954 & 27.416 & 61.552 & 154.63 & 155.22 & 87.58 & 206.18 & 256.60 & 278.43 & 284.63 & 361.56 \\
\hline & 3 & 152.04 & 60.878 & 92.824 & 211.79 & 220.50 & 118.40 & 214.97 & 283.89 & 309.74 & 357.24 & 422.31 \\
\hline & 4 & 209.65 & 78.043 & 122.02 & 282.40 & 243.01 & 204.83 & 293.09 & 376.96 & 309.83 & 408.30 & 493.91 \\
\hline & 5 & 279.78 & 136.49 & 176.11 & 301.79 & 261.11 & 210.19 & 361.44 & 378.75 & 377.56 & 460.47 & 515.13 \\
\hline
\end{tabular}




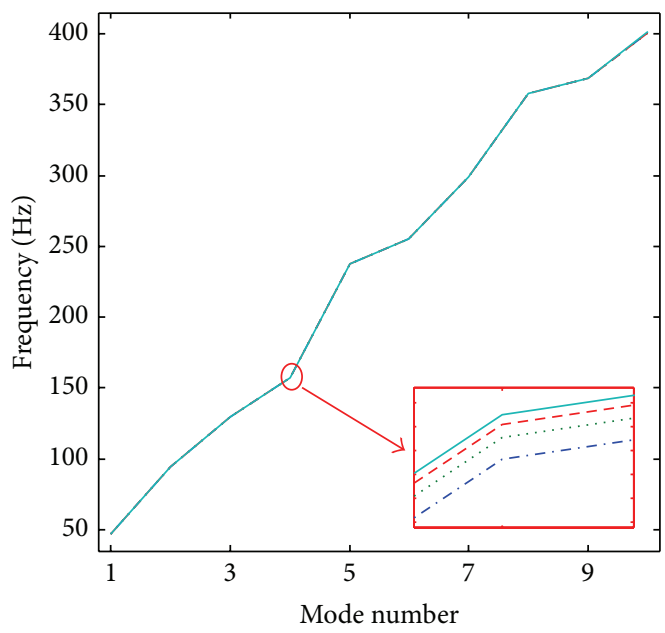

(a) FFFF

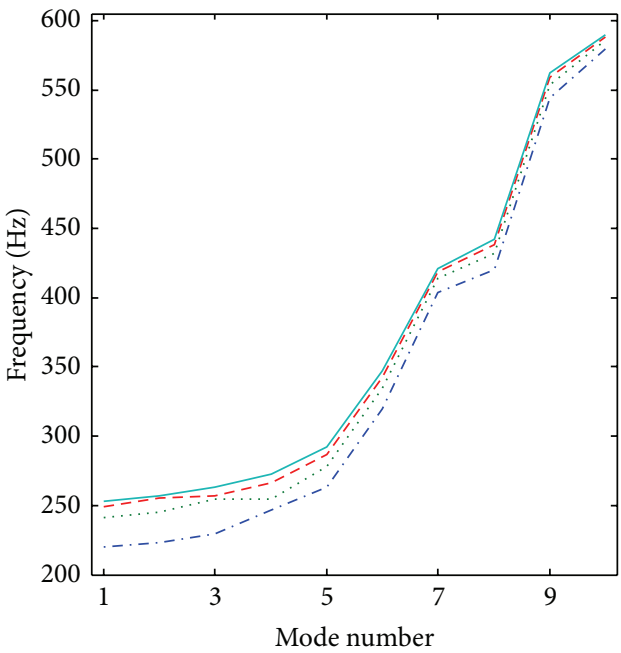

(c) $\mathrm{CFFF}$

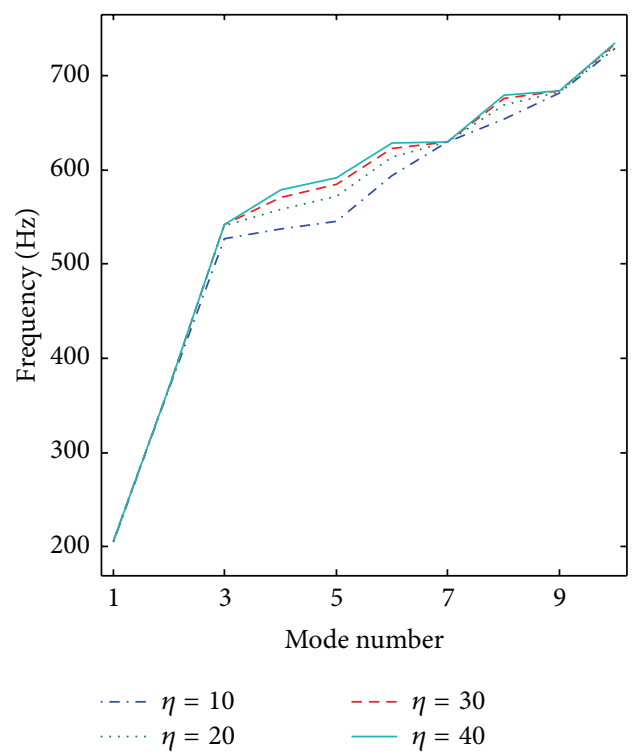

(e) SSSS

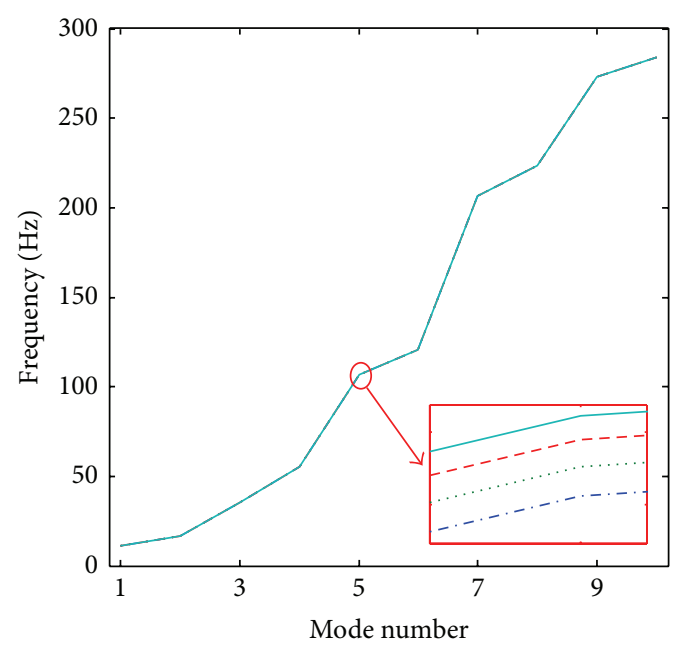

(b) FFFC

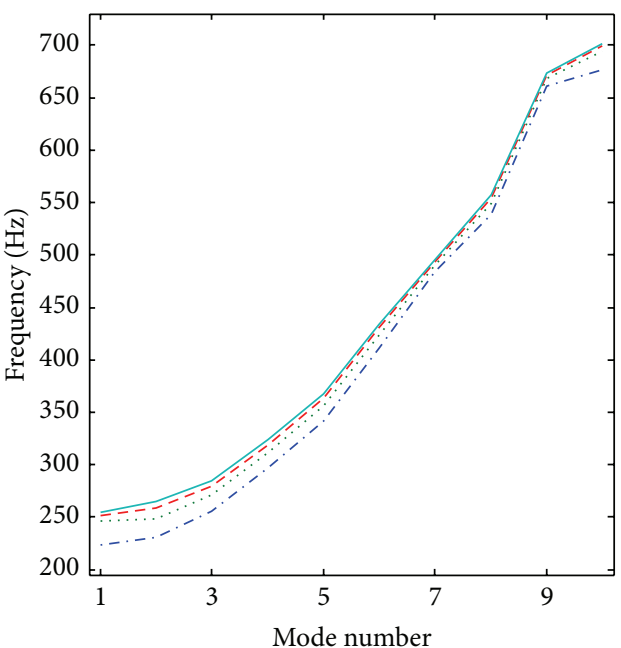

(d) $\mathrm{CCFF}$

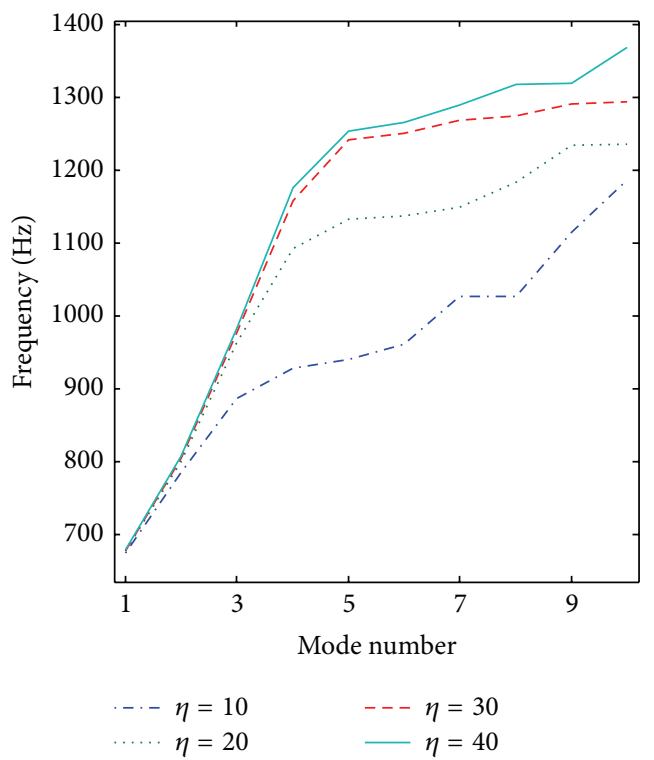

(f) CCCC

FIGURE 10: Variation of the first ten frequencies $(\mathrm{Hz})$ against material parameter $\eta\left(\eta=E_{11} / E_{22}\right)$ for an open spherical shell with different boundary conditions. 


\section{Acknowledgment}

The authors gratefully acknowledge the financial support from the National Natural Science Foundation of China (nos. 51175098 and 51279035).

\section{References}

[1] A. Selmane and A. A. Lakis, "Dynamic analysis of anisotropic open cylindrical shells," Computers and Structures, vol. 62, no. 1, pp. 1-12, 1997.

[2] E. Bahmyari, M. M. Banatehrani, M. Ahmadi, and M. Bahmyari, "Vibration analysis of thin plates resting on Pasternak foundations by element free Galerkin method," Shock and Vibration, vol. 20, no. 2, pp. 309-326, 2013.

[3] T. Ye, G. Jin, Y. Chen, and S. Shi, "A unified formulation for vibration analysis of open shells with arbitrary boundary conditions," International Journal of Mechanical Sciences, vol. 81, pp. 42-59, 2014.

[4] Ö. Civalek, "Vibration analysis of conical panels using the method of discrete singular convolution," Communications in Numerical Methods in Engineering, vol. 24, no. 3, pp. 169-181, 2008.

[5] L. Zhang and Y. Xiang, "Vibration of open circular cylindrical shells with intermediate ring supports," International Journal of Solids and Structures, vol. 43, no. 13, pp. 3705-3722, 2006.

[6] Y. Qu, S. Wu, Y. Chen, and H. Hua, "Vibration analysis of ringstiffened conical-cylindrical-spherical shells based on a modified variational approach," International Journal of Mechanical Sciences, vol. 69, pp. 72-84, 2013.

[7] M. S. Qatu and E. Asadi, "Vibration of doubly curved shallow shells with arbitrary boundaries," Applied Acoustics, vol. 73, no. 1, pp. 21-27, 2012.

[8] S. Bashmal, R. Bhat, and S. Rakheja, "In-plane free vibration analysis of an annular disk with point elastic support," Shock and Vibration, vol. 18, no. 4, pp. 627-640, 2011.

[9] X. Zhao, T. Y. Ng, and K. M. Liew, "Free vibration of twoside simply-supported laminated cylindrical panels via the mesh-free kp-Ritz method," International Journal of Mechanical Sciences, vol. 46, no. 1, pp. 123-142, 2004.

[10] B. Liu, Y. F. Xing, M. S. Qatu, and A. J. M. Ferreira, "Exact characteristic equations for free vibrations of thin orthotropic circular cylindrical shells," Composite Structures, vol. 94, no. 2, pp. 484-493, 2012.

[11] A. W. Leissa, "Vibration of shells," NASA SP-288, Government Printing Office, Washington, DC, USA, 1973.

[12] J. N. Reddy, "Exact solutions of moderately thick laminated shells," Journal of Engineering Mechanics, vol. 110, no. 5, pp. 794$809,1984$.

[13] K. M. Liew and C. W. Lim, "A Ritz vibration analysis of doubly-curved rectangular shallow shells using a refined firstorder theory," Computer Methods in Applied Mechanics and Engineering, vol. 127, no. 1-4, pp. 145-162, 1995.

[14] J. N. Reddy and C. F. Liu, "A higher-order shear deformation theory of laminated elastic shells," International Journal of Engineering Science, vol. 23, no. 3, pp. 319-330, 1985.

[15] T. Ye, G. Jin, Z. Su, and X. Jia, "A unified Chebyshev-Ritz formulation for vibration analysis of composite laminated deep open shells with arbitrary boundary conditions," Archive of Applied Mechanics, vol. 84, no. 4, pp. 441-471, 2014.
[16] A. Selmane and A. A. Lakis, "Vibration analysis of anisotropic open cylindrical shells subjected to a flowing fluid," Journal of Fluids and Structures, vol. 11, no. 1, pp. 111-134, 1997.

[17] E. Asadi, W. Wang, and M. S. Qatu, "Static and vibration analyses of thick deep laminated cylindrical shells using 3D and various shear deformation theories," Composite Structures, vol. 94, no. 2, pp. 494-500, 2012.

[18] A. Bhimaraddi, "A higher order theory for free vibration analysis of circular cylindrical shells," International Journal of Solids and Structures, vol. 20, no. 7, pp. 623-630, 1984.

[19] F. Tornabene, A. Liverani, and G. Caligiana, "FGM and laminated doubly curved shells and panels of revolution with a free-form meridian: a 2-D GDQ solution for free vibrations," International Journal of Mechanical Sciences, vol. 53, no. 6, pp. 446-470, 2011.

[20] C. T. Loy and K. Y. Lam, "Vibration of thick cylindrical shells on the basis of three-dimensional theory of elasticity," Journal of Sound and Vibration, vol. 226, no. 4, pp. 719-737, 1999.

[21] K. M. Liew, L. X. Peng, and T. Y. Ng, “Three-dimensional vibration analysis of spherical shell panels subjected to different boundary conditions," International Journal of Mechanical Sciences, vol. 44, no. 10, pp. 2103-2117, 2002.

[22] C. W. Lim, K. M. Liew, and S. Kitipornchai, "Vibration of open cylindrical shells: a three-dimensional elasticity approach," Journal of the Acoustical Society of America, vol. 104, no. 3, pp. 1436-1443, 1998.

[23] K. M. Liew, L. A. Bergman, T. Y. Ng, and K. Y. Lam, “Threedimensional vibration of cylindrical shell panels-solution by continuum and discrete approaches," Computational Mechanics, vol. 26, no. 2, pp. 208-221, 2000.

[24] A. W. Leissa and J.-H. Kang, “Three-dimensional vibration analysis of thick shells of revolution," Journal of Engineering Mechanics, vol. 125, no. 12, pp. 1365-1371, 1999.

[25] J. So and A. W. Leissa, "Free vibrations of thick hollow circular cylinders from three-dimensional analysis," Journal of Vibration and Acoustics, vol. 119, no. 1, pp. 89-95, 1997.

[26] J.-H. Kang and A. W. Leissa, "Three-dimensional vibrations of hollow cones and cylinders with linear thickness variations," The Journal of the Acoustical Society of America, vol. 106, no. 2, pp. 748-755, 1999.

[27] D. Zhou, Y. K. Cheung, S. H. Lo, and F. T. K. Au, “3D vibration analysis of solid and hollow circular cylinders via ChebyshevRitz method," Computer Methods in Applied Mechanics and Engineering, vol. 192, no. 13-14, pp. 1575-1589, 2003.

[28] D. Zhou and S. H. Lo, "Three-dimensional free vibration analysis of doubly-curved shells," Journal of Vibration and Control, 2013.

[29] M. R. Mofakhami, H. H. Toudeshky, and S. H. Hashemi, "Finite cylinder vibrations with different end boundary conditions," Journal of Sound and Vibration, vol. 297, no. 1-2, pp. 293-314, 2006.

[30] S. M. R. Khalili, A. Davar, and K. Malekzadeh Fard, "Free vibration analysis of homogeneous isotropic circular cylindrical shells based on a new three-dimensional refined higher-order theory," International Journal of Mechanical Sciences, vol. 56, no. 1, pp. 1-25, 2012.

[31] W. Q. Chen, Z. G. Bian, and H. J. Ding, "Three-dimensional vibration analysis of fluid-filled orthotropic FGM cylindrical shells," International Journal of Mechanical Sciences, vol. 46, no. 1, pp. 159-171, 2004. 
[32] J. N. Sharma, "Three-dimensional vibration analysis of a homogeneous transversely isotropic thermoelastic cylindrical panel," The Journal of the Acoustical Society of America, vol. 110, no. 1, pp. 254-259, 2001.

[33] Y. Qu and G. Meng, "Three-dimensional elasticity solution for vibration analysis of functionally graded hollow and solid bodies of revolution. Part I: theory," European Journal of Mechanics A: Solids, vol. 44, pp. 222-233, 2014.

[34] P. Malekzadeh, M. Farid, P. Zahedinejad, and G. Karami, "Three-dimensional free vibration analysis of thick cylindrical shells resting on two-parameter elastic supports," Journal of Sound and Vibration, vol. 313, no. 3-5, pp. 655-675, 2008.

[35] A. W. Leissa and M. S. Qatu, Vibrations of Continuous Systems, McGraw-Hill, New York, NY, USA, 2011.

[36] M. S. Qatu, Vibration of Laminated Shells and Plates, Elsevier, San Diego, Calif, USA, 2004.

[37] J. N. Reddy, Mechanics of Laminated Composite Plates and Shells: Theory and Analysis, CRC Press, New York, NY, USA, 2nd edition, 2004.

[38] A. S. Saada, Elasticity: Theory and Applications, Ross Publishing, Plantation, Fla, USA, 2nd edition, 2009.

[39] M. S. Qatu, "Recent research advances in the dynamic behavior of shells: 1989-2000, part 1: laminated composite shells," Applied Mechanics Reviews, vol. 55, no. 4, pp. 325-349, 2002.

[40] M. S. Qatu, "Recent research advances in the dynamic behavior of shells: 1989-2000, Part 2: homogeneous shells," Applied Mechanics Reviews, vol. 55, no. 5, pp. 415-434, 2002.

[41] K. P. Soldatos, "Review of three-dimensional dynamic analyses of circular cylinders and cylindrical shells," Applied Mechanics Reviews, vol. 47, no. 10, pp. 501-516, 1994.

[42] W. L. Li, "Free vibrations of beams with general boundary conditions," Journal of Sound and Vibration, vol. 237, no. 4, pp. 709-725, 2000.

[43] H. Khov, W. L. Li, and R. F. Gibson, "An accurate solution method for the static and dynamic deflections of orthotropic plates with general boundary conditions," Composite Structures, vol. 90, no. 4, pp. 474-481, 2009.

[44] G. Jin, Z. Su, S. Shi, T. Ye, and S. Gao, "Three-dimensional exact solution for the free vibration of arbitrarily thick functionally graded rectangular plates with general boundary conditions," Composite Structures, vol. 108, no. 1, pp. 565-577, 2014.

[45] K. M. Liew, K. C. Hung, and M. K. Lim, "A continuum threedimensional vibration analysis of thick rectangular plates," International Journal of Solids and Structures, vol. 30, no. 24, pp. 3357-3379, 1993.

[46] K. M. Liew, K. C. Hung, and M. K. Lim, "Free vibration studies on stress-free three-dimensional elastic solids," Journal of Applied Mechanics, vol. 62, no. 1, pp. 159-165, 1995. 

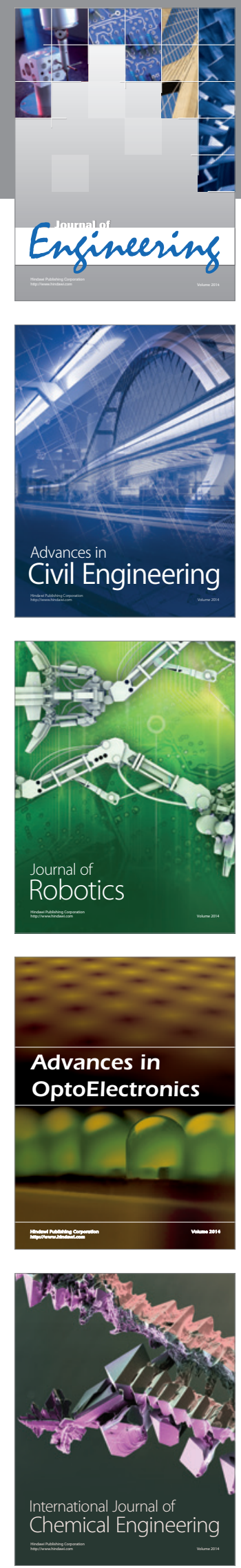

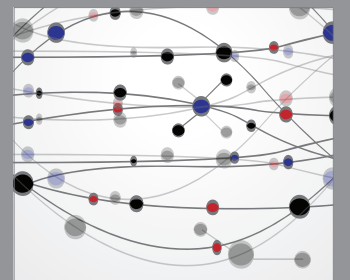

The Scientific World Journal
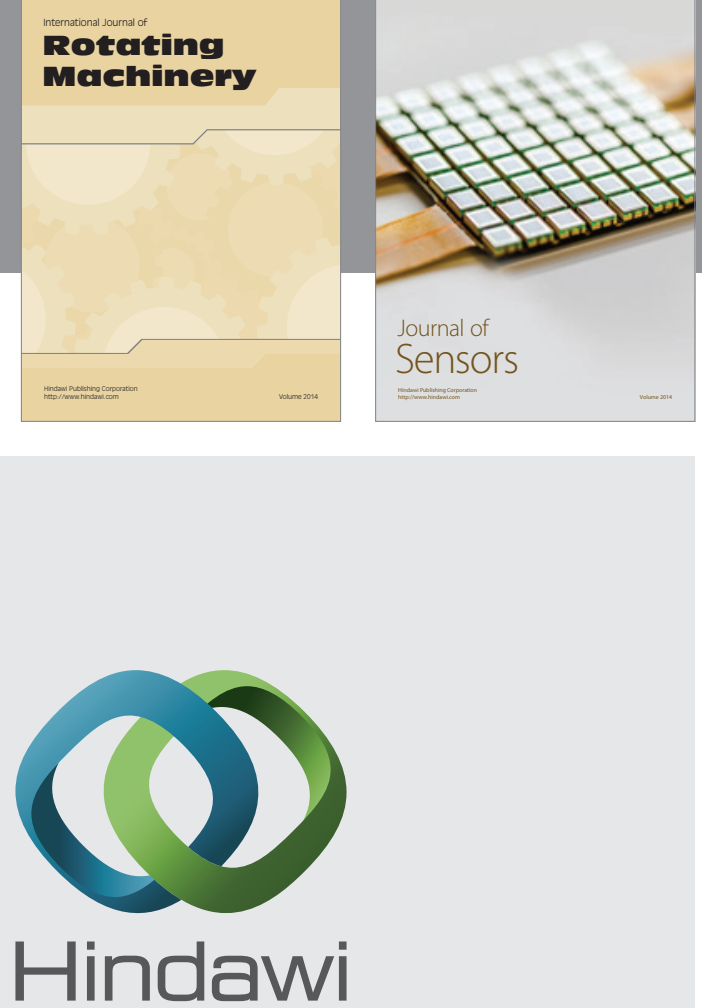

Submit your manuscripts at http://www.hindawi.com
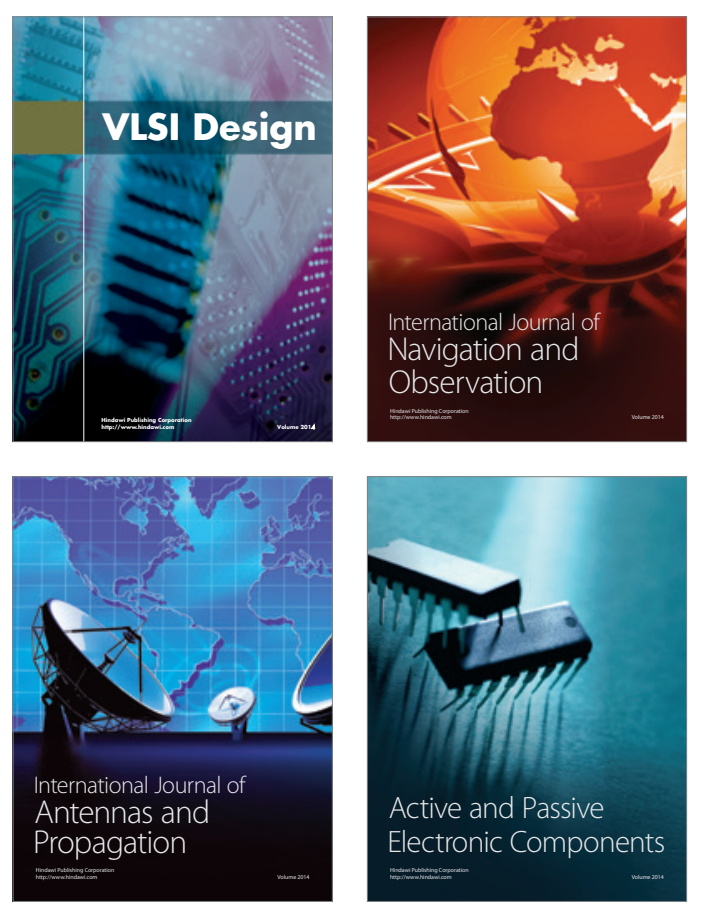
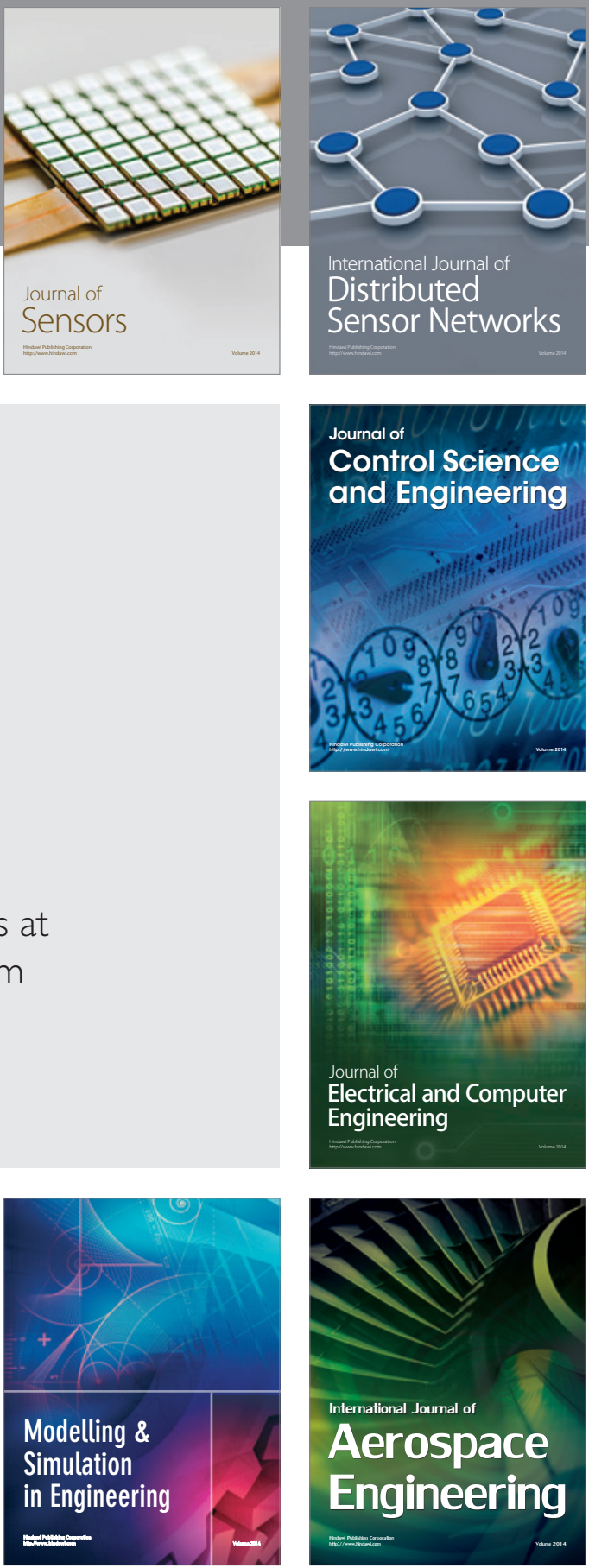

Journal of

Control Science

and Engineering
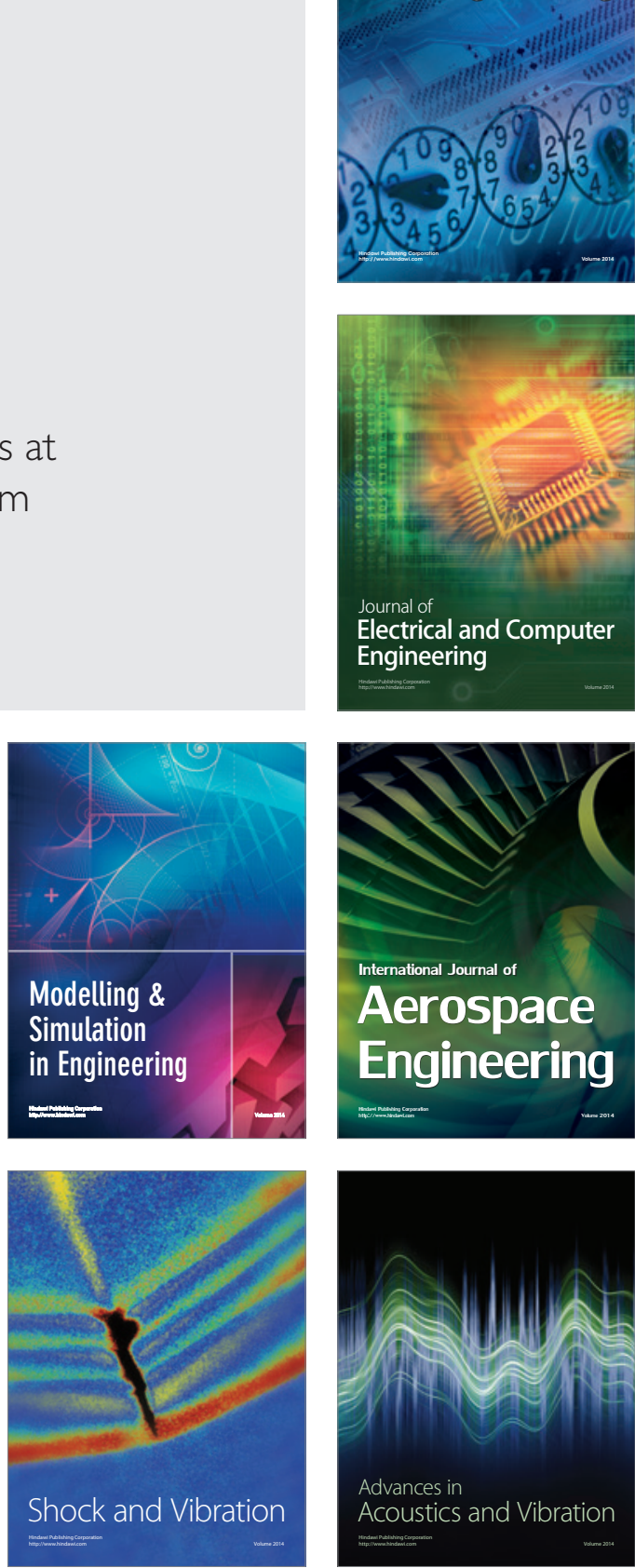